\title{
Modal Analysis of Vertical Array Receptions for the Heard Island Feasibility Test
}

\author{
by \\ Brian J. Sperry
}

B.S., Electrical and Computer Engineering, University of Iowa (1990)

Submitted in partial fulfillment of the

requirements for the degree of

\begin{abstract}
MASTER OF SCIENCE IN OCEANOGRAPHIC ENGINEERING
\end{abstract}

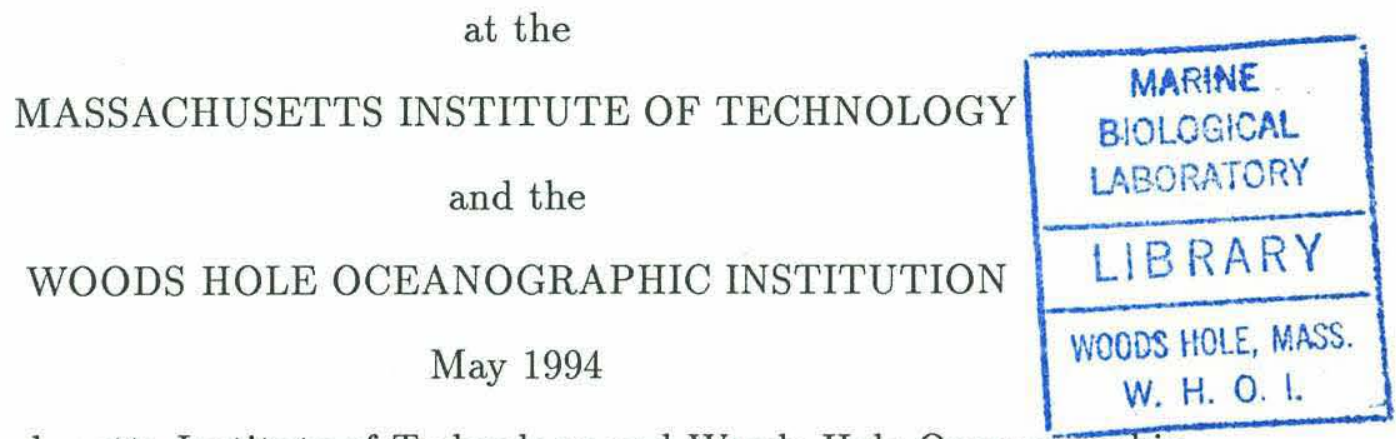

(c) Massachusetts Institute of Technology and Woods Hole Oceanographic

Institution, 1994

All rights reserved.

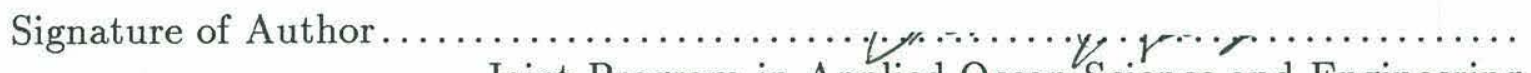

Joint Program in Applied Ocean Science and Engineering Massachusetts Institute of Technology Woods Hole Oceanographic Institution $\cap \ldots$ May 27, 1994

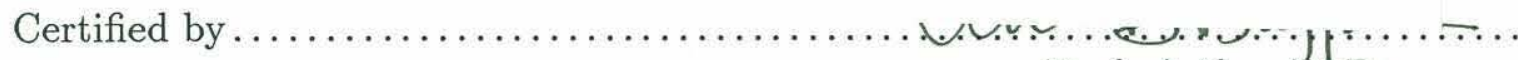

Prof. Arthur B. Baggeroer Massachusetts Institute of Technology 1 Thesis Supervisor

Accepted by

Prof. Arthur B. Blaggeroer Chairman, Joint Committee forngered Ocean Science and Engineering Massachusetts Institute of Tech plogy/Nood Hole Oceanographic Institution 



\title{
Modal Analysis of Vertical Array Receptions for the Heard Island Feasibility Test
}

\author{
by Brian J. Sperry
}

\author{
Submitted to the Massachusetts Institute of Technology/ \\ Woods Hole Oceanographic Institution \\ Joint Program in Applied Ocean Science and Engineering \\ on February 18, 1994, in partial fulfillment of the requirements \\ for the degree of Master of Science in Ocean Engineering
}

\begin{abstract}
During the 1991 Heard Island Feasibility Test, a vertical hydrophone array deployed off Monterey, CA, recorded transmissions from a low-frequency acoustic source nearly $18,000 \mathrm{~km}$ away. By determining the modal structure of the received transmissions, it is possible to characterize the physics of such long range propagation. This thesis focuses on the determination of the modal, or vertical, structure of the signal. It was necessary to first develop a conditioning scheme to address several data quality issues, including very low signal levels ( $-15 \mathrm{~dB}$ SNR on a single channel), large transient spikes, and a limited set of operational channels. Very narrowband filtering was used to obtain a $25 \mathrm{~dB}$ increase in SNR. Doppler shifts for each transmission event were predicted from available parameters and were found to be within $\pm 2 \mathrm{mHz}$ of the measured shifts.

The modal analysis employed two methods: comparing variations in signal energy with depth to the vertical extent of the modes, and fitting the data using a least squares modal decomposition. The least squares performance given a subsampled basis set of modes was studied and improved upon through the use of diagonal loading. Lack of array orientation data hindered the analysis, and least squares fitting was used to estimate the most likely orientation. The least squares analysis indicated the presence of modes at least up to mode 7, possibly higher. This is significant in that predictions prior to the experiment were that all but the lowest modes would be attenuated by boundary interactions along the path. Results from independent analyses of the same data also support the conclusion that the signal structure is quite complex.
\end{abstract}

Thesis Supervisor: Prof. Arthur B. Baggeroer Massachusetts Institute of Technology 


\section{Acknowledgments}

I would like to first acknowledge my advisor and thesis supervisor, Prof. Arthur Baggeroer. His guidance with this work has been invaluable, and his patience with my learning curve deeply appreciated. Many thanks to my fellow colleagues Kathleen Wage and Peter Traykovski for their helpful discussions, support, and proof reading. A special thanks to those at WHOI - Eddie Scheer for his help with the computers and Keith von der Heydt for his information on the technical details of the array. Also, what thesis experience would be complete without a bunch of officemates to thank - Joe, Matt, Tarun, Dave, Diane, and the rest of the 5-435 crowd.

Many thanks to my parents, brothers, and grandparents for their continued interest and support. I am particularly grateful for their enthusiasm regarding my work.

Finally, and most importantly, I wish to thank my wife Kelly Poort, not only for putting up with my long hours and extended stays in Woods Hole, but also for providing words of encouragement, motivation, and understanding at just the right moments. I certainly could not have made it this far without you, Kel!

The Vertical Line Array effort for the Heard Island Feasibility Test was supported by the Monterey Bay Aquarium Research Institute (MBARI), the Naval Postgraduate School (NPS), and Science Applications International Corporation (SAIC). Funding for this work was provided by the Department of Energy Grant \#DE-FG0291ER61100 (Vertical Arrays for the Heard Island Acoustic Feasibility Experiment

on Monitoring Global Warming), and the University of California - Scripps ATOC Agreement, PO\#10037359. 


\section{Contents}

Table of Contents $\quad 5$

$\begin{array}{ll}\text { List of Tables } & 7\end{array}$

$\begin{array}{lr}\text { List of Figures } & 8\end{array}$

1 Introduction $\quad 11$

1.1 Introduction . . . . . . . . . . . . . . . . 11

1.2 The Monterey Vertical Array . . . . . . . . . . . . . . . 13

1.3 Acoustic Propagation and

Normal Mode Theory . . . . . . . . . . . . . . . . . . . 15

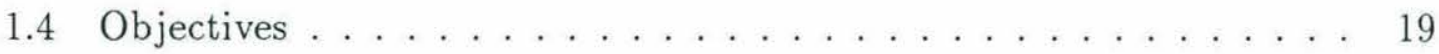

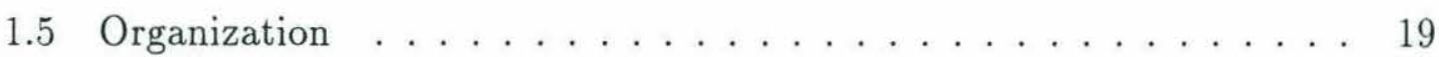

2 Preliminary Processing $\quad 21$

2.1 First Stage . . . . . . . . . . . . . . . . . . 23

2.1.1 Spike Removal . . . . . . . . . . . . . . . . . . . . 23

2.1.2 Demodulation and Decimation ............ 23

2.2 Doppler Analysis . . . . . . . . . . . . . . . . . . . 26

2.2.1 Prediction ........................ 26

2.2.2 Measurement ................... 27 


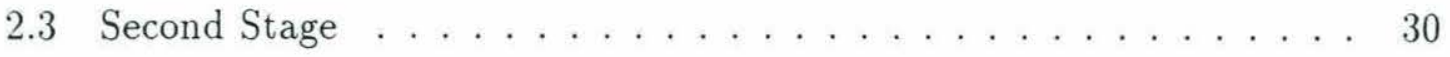

2.4 Preliminary Analysis . . . . . . . . . . . . . . . . . 31

3 Least Squares Modal Beamforming $\quad 39$

3.1 Formulation . . . . . . . . . . . . . . . . . . . . 41

3.2 Diagonal Loading . . . . . . . . . . . . . . . . . . 48

3.3 Determining the number of modes . . . . . . . . . . 51

3.4 Performance . . . . . . . . . . . . . . . . . . 52

4 Modal Analysis $\quad 61$

4.1 Normal Mode Data . . . . . . . . . . . . . . . . . . . . . . . 61

4.2 Sonograms . . . . . . . . . . . . . . . . . . . 62

4.3 Array Geometry . . . . . . . . . . . . . . . . . . . . . 73

4.4 Modal Fitting . . . . . . . . . . . . . . . . . . . 79

4.5 Interpretation $\ldots \ldots \ldots \ldots \ldots \ldots \ldots \ldots \ldots \ldots \ldots \ldots \ldots$

5 Conclusions $\quad 85$

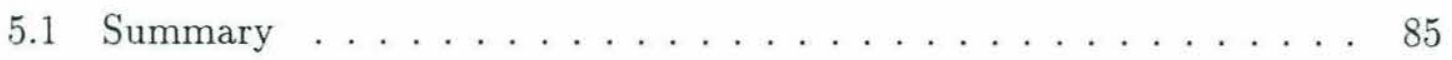

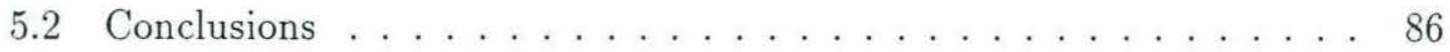

5.3 Future Work . . . . . . . . . . . . . . . 87

$\begin{array}{lr}\text { Bibliography } & 89\end{array}$ 


\section{List of Tables}

2.1 Summary of transmission events selected for analysis. . . . . . . . . . 22

2.2 Comparison of predicted and measured Doppler shift for the CW signals received on the VLA. . . . . . . . . . . . . . . . 27

2.3 Signal statistics for Event 01261525 for the time period 0 - 2500 seconds. 33

2.4 Signal statistics for Event 01270322 for the time period $1000-3000$ seconds. . . . . . . . . . . . . . . . . 33

2.5 Signal statistics for Event 01271505 for the time period $1000-3000$ seconds. . . . . . . . . . . . . . . . . . 34

3.1 Modal covariance eigenvalues, their relative percentages, and the relative percentages after loading. . . . . . . . . . . . 50 50

4.1 Estimated tilt and azimuth angles based on estimation-fit. . . . . . . 78 


\section{List of Figures}

1-1 Paths taken by sound in the Heard Island Feasibility Test. . . . . . . 14

1-2 Deployment configuration of vertical array off Monterey. . . . . . . . 16

2-1 Processing flowchart . . . . . . . . . . . . . . . 22

2-2 Frequency response for the $45-75 \mathrm{~Hz}$, Parks-McClellan design, bandpass filter . . . . . . . . . . . . . . . . . . . . 24

2-3 Spectogram of 01261525, Channel 0 data after bandpass filtering and clipping. . . . . . . . . . . . . . . . . . 24

2-4 Frequency response for the $\pm 35 \mathrm{mHz}$, Parks-McClellan lowpass filter. 25

2-5 Sonogram - Channel 0, Event 01261525. . . . . . . . . . . . 28

2-6 Sonogram - Channel 0, Event 01270322. . . . . . . . . . . . . 29

2-7 Sonogram - Channel 0, Event 01271505. . . . . . . . . . . . . . 29

2-8 Frequency response for the $\pm 5 \mathrm{mHz}$ lowpass filter. . . . . . . . . 30

2-9 Magnitude and phase for Event 01271525. . . . . . . . . . . 35

2-10 Magnitude and phase for Event 01270322. . . . . . . . . . . . 36

2-11 Magnitude and phase for Event 01271505. . . . . . . . . . . . . 37

2-12 Total power across the array for the three CW transmissions. . . . . . 38

3-1 Covariance matrix for modes 1-12, computed using only the top 14

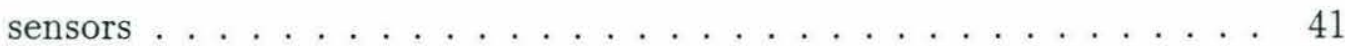


3-2 Least squares and single-mode beamformer responses for steering modes $1-7$, and data channels $1-14 \ldots . . \ldots 4 . \ldots 46$

3-3 Response power for steering modes 1-7. . . . . . . . . . . . . 47

3-4 Total power for each mode coefficient for various levels of diagonal loading of the least squares processor. . . . . . . . . . . . 51

3-5 Singularity coefficients for the modal covariance matrix $\mathbf{K}_{\phi}$ at various levels of diagonal loading. . . . . . . . . . . . . . . . 53

3-6 Normalized least squares beamformer response to synthetic data with $20 \mathrm{~dB}$ SNR. . . . . . . . . . . . . . . . . 56

3-7 Normalized least squares beamformer response to synthetic data with $10 \mathrm{~dB}$ SNR. . . . . . . . . . . . . . . . . . 57

3-8 Normalized least squares beamformer response to synthetic data with $0 \mathrm{~dB}$ SNR. . . . . . . . . . . . . . . . . . . . 58

3-9 Estimation fits from synthetic data. . . . . . . . . . . . 59

4-1 Sound speed profile and computed modeshapes at the Monterey site. 62

4-2 Sonograms from hydrophones $1-6$ on the VLA. . . . . . . . . . . 65

4-3 Sonograms from hydrophones 7-12 on the VLA. . . . . . . . . . . 67

4-4 Sonograms from hydrophones $13,14,18,20,23$, and 24 on the VLA. 69

4-5 Sonograms from hydrophones 27,28 , and 30 on the VLA. . . . . . . 71

4-6 Output from upper tilt sensor. . . . . . . . . . . . . . . . . . 74

4-7 Output from upper depth sensor. . . . . . . . . . . . 75

4-8 Schematic of array orientation. . . . . . . . . . . . . . 75

4-9 Difference between upper and lower depth sensors. . . . . . . . . . . 76

4-10 Event 01261525 estimation fit based on 7-mode least squares fit averaged over the period $0-2000$ seconds. . . . . . . . . . . . . . 77

4-11 Event 01270322 estimation fit based on 7-mode least squares fit averaged over the period 1600-2400 seconds. . . . . . . . . . . . 77 
4-12 Event 01271505 estimation fit based on 7-mode least squares fit averaged over the period 1000-2500 seconds. . . . . . . . . . . 78

4-13 Estimated mode coefficients for Event 01261525. . . . . . . . . . . . 82

4-14 Estimated mode coefficients for Event 01270322. . . . . . . . . . . . 83

4-15 Estimated mode coefficients for Event 01271505. . . . . . . . . . . . 84 


\section{Chapter 1}

\section{Introduction}

\subsection{Introduction}

Global climate change, in particular the possibility of climate change of anthropogenic origin, is a topic of increasing concern. In response to this concern, Munk and Forbes suggested in 1989 the possibility of monitoring changes in global ocean temperatures by measuring changes in travel times of acoustic signals transmitted across entire ocean basins [1]. The proposed monitoring scheme may be divided into two main components: the use of acoustics to resolve the large-scale temperature structure of the oceans and the subsequent identification of long-term climate trends against the background of natural gyre and basin scale variability. Preliminary analyses indicate the latter will be the more difficult task [3]. The motivation for considering changes in ocean temperature may be explained as follows. If the ocean temperature structure is decomposed into temporal and spatial empirical orthogonal functions (EOF's), the greenhouse signal and the natural variability are expected to occupy different EOF's, and are therefore separable. This is the key advantage of considering the ocean rather than the atmosphere, where climate trends and background variability are on the same time and space scales [2]. Even with 
this advantage, it is estimated that ten years of study will be necessary before any long-term trends are evident.

The other component of the monitoring scheme, the use of acoustics for determining ocean temperatures is called acoustic thermometry. This is an ideal method for obtaining large-scale average temperatures since the long propagation paths average out the travel time perturbations of smaller meso-scale features, such as eddies. The use of such long ranges, however, presents numerous technical challenges. For instance, it was known from a 1960 experiment that sound from an explosive source could be detected nearly halfway around the world [4]. However, an explosive charge does not provide the repeatable source signal necessary for acoustic thermometry work, and sidelobes from resulting bubble oscillations make accurate travel time determination difficult. To obtain the necessary resolution, on the order of 10-50 msecs, a hydroacoustic source is required. The longest ranges that these sources have been used are 4000 kilometers [5], whereas basin-scale ranges are 10-18 megameters (1 megameter $=10^{6}$ meters). In addition to uncertainties regarding the use of hydroacoustic sources, it was not known what effects the long propagation paths would have on the acoustic signal structure. In particular, was it possible to identify and track the individual multipath arrivals over repeated transmissions? In an effort to resolve some of the more pressing issues, the Heard Island Feasibility Test (HIFT) was conducted in January of 1991. The experiment confirmed that it was indeed feasible to use a hydroacoustic source and that signals could be coherently processed to obtain travel times at ranges up to $18,000 \mathrm{~km}$. As part of the experiment, both vertical and horizontal line arrays were deployed in an effort to determine the spatial characteristics of the arriving signals. The processing and analysis of the receptions on a vertical array deployed off the coast of California form the basis for this thesis. The vertical distribution of the signal holds crucial information on the propagation characteristics. Understanding how the signals propagate is fundamental to being 
able to extract the necessary climate information.

The HIFT was a collaborative effort between many institutions, including Scripps Institute of Oceanography, MIT, Woods Hole Oceanographic Institute, University of Washington, University of Michigan, Science Applications International Corporation (SAIC), Naval Research Lab and Hubbs Sea World. Transmissions took place from a source ship just off Heard Island in the Southern Indian Ocean $\left(54^{\circ} S, 74^{\circ} E\right)$. Low frequency signals were transmitted for one hour, every three hours, for five days. Fourteen different receiving sites were located around the world. Figure 1-1 shows the experimental deployment. The following section briefly discusses the Monterey vertical array. A more detailed accounting of the experiment may be found in the HIFT overview paper by Munk, Spindel, Baggeroer, and Birdsall [3].

\subsection{The Monterey Vertical Array}

Two identical vertical arrays were deployed for the HIFT, one off Bermuda and the other off the coast of California. Unfortunately, the Bermuda array sank and no data were obtained. Much effort was put into selecting locations along the West coast which could reliably receive transmissions from Heard Island. Acoustic propagation modeling carried out by Chiu, et al. [6], used gridded temperature and salinity data provided by a global circulation model as input to the HARPO ${ }^{1}$ ray tracing code. Using the computed ray paths, only one possible region was found - a narrow band of insonification off the California coast, approximately $150 \mathrm{~km}$ wide [6]. During the experiment, the array was positioned within this envelope, about $200 \mathrm{~km}$ offshore and in deep water free of any significant bathymetry.

Figure 1-2 shows the configuration the array. There were 32 sensors spaced 45 meters apart. Nominal depth of the first hydrophone was 345 meters. This placed

\footnotetext{
${ }^{1}$ Hamiltonian Acoustic Ray tracing Program for the Ocean.
} 


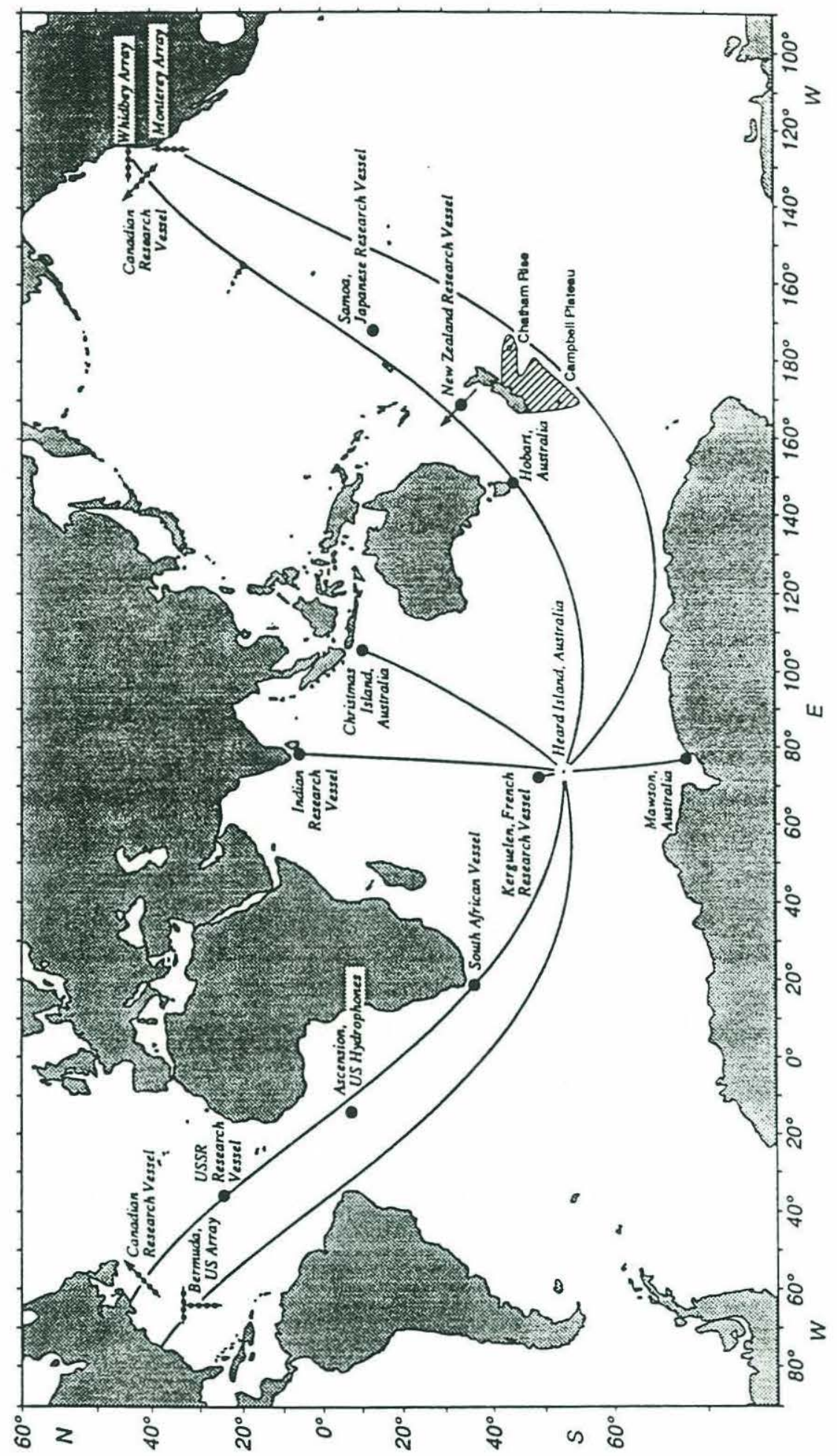

Figure 1-1: Paths taken by sound in the Heard Island Feasibility Test. Black circles indicate receiver sites. Horizontal lines represent horizontal receiver arrays. Vertical lines designate vertical arrays. Lines with arrows indicate towed arrays. Signals were received at all sites except for the Japanese station off Samoa. See reference [1]. 
the sound speed axis between hydrophones 5 and 6 . Each hydrophone on the VLA had a sensitivity of $-170 \mathrm{~dB}$ re $1 \mathrm{~V} / \mu \mathrm{Pa}$. To appreciate such a sensitivity, consider that a $3 \mathrm{~cm}$ vertical displacement of a single hydrophone would produce a 1 volt output, nearly 10,000 times the output from the actual signal. Because of this, great care was taken to isolate the array from surface heave. Extensive damping kept swell-induced array movement under $15 \mathrm{~cm}$, preventing saturation of the data acquisition equipment. A more detailed discussion of the array design is in the paper by Baggeroer, et al. [7].

The primary reason for deploying vertical arrays was to resolve the arriving signal structure in order to learn more about the characteristics of very long range propagation. A brief overview of the more important considerations in long range propagation is given in the next section, and in particular, a discussion of normal mode theory, which is useful for representing the propagation of an acoustic signal.

\subsection{Acoustic Propagation and Normal Mode Theory}

When working with megameter or greater propagation distances, many assumptions and approximations that can be made for shorter distances are no longer valid. For instance, the curvature of the earth must be considered when computing the horizontal ray paths, as well as refraction due to horizontal temperature gradients and changing bathymetry. The paths shown in Figure 1-1 are actually refracted geodesics. A good discussion of horizontal refraction is given by Heaney, et al. [8]. Another consideration in long range propagation is the use of low frequencies to minimize volume attenuation, or absorption losses. As an example, at $57 \mathrm{~Hz}$, the 


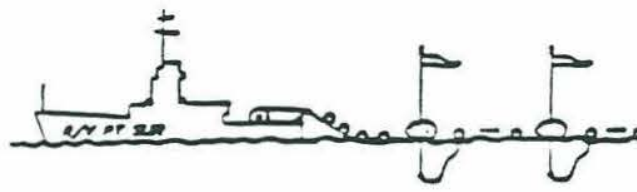

Moss Landing Marine Labs R/V Pt. Sur
4 Markers @ 300m "Spring" Floats 2m spacing
Syntactic Foam Float

32 Hydrophones 45 meter spacing

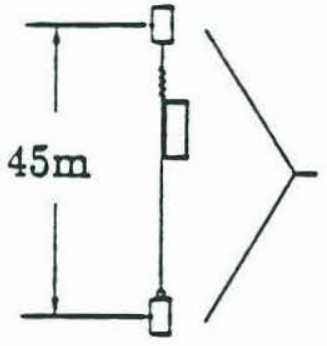

Pressure

Tilt Sensors

Total cable length $=3100 \mathrm{~m}$ 10000 lb Kevlar core 50 twisted pair SAIC "Quiet Cable" coating
Acoustic Release

Sinker/Damper
Distance from Surface

$30 m$

$230 m$

$345 m$

$1740 \mathrm{~m}$
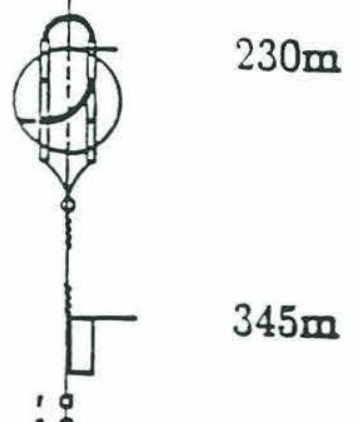

28

3

: SAIC "Quiet Cable" coating

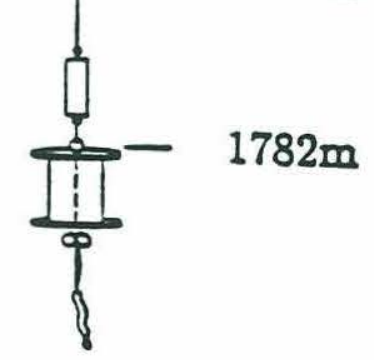

Figure 1-2: Deployment configuration of vertical array off Monterey [7]. 
attenuation over an $18,000 \mathrm{~km}$ path in the Atlantic would be about $3 \mathrm{~dB}^{2}$. At 100 $\mathrm{Hz}$, though, the loss jumps to $19 \mathrm{~dB}$.

At these low frequencies, a useful and well-established method for describing the long range propagation of acoustic signals is a normal mode representation. Intuitively, a normal mode may be thought of as the coherent interference of a system of rays all having the same horizontal wavenumber, or phase speed [9]. The vertical extent of a particular mode is determined by the turning depths of the component rays, or the depths at which the local sound speed equals the phase speed of the mode/rays. This has the important consequence that higher-order modes sample more of the water column and thus each mode may contain slightly different information about the ocean. A receiver that can resolve individual modes can potentially make inferences about how ocean properties, such as temperature, vary with depth.

The use of normal modes lends itself well to an efficient expression for the sound field at a particular depth and range. The received signal may be expressed as a weighted superposition of the normal modes, plus an appropriate expression for the noise field. At a depth $z$ and range $r$ from the source, the field may be written as

$$
p(r, z)=\sum_{i} a_{i}\left(z_{o}\right) \phi_{i}(r, z) R_{i}(r)+n(r, z)
$$

where $a_{i}$ describes how each mode is excited by the source, $\phi_{i}$ is the $i^{\text {th }}$ modeshape at the location $(r, z)$, and all of the range information is expressed in $R_{i}$. For long ranges, the number of modes required in the summation is relatively small, due to the fact that the higher modes are attenuated by boundary interactions along the path. Barring re-population of the higher modes, then, only the lowest few would be expected to be present after $18,000 \mathrm{~km}$ of propagation.

\footnotetext{
${ }^{2}$ For Pacific waters, the loss would be about $5 \mathrm{~dB}$.
} 
Since there are only a small number of modes to consider, and since the vertical array provides a discrete sampling of the sound field, Eq. (1.1) may be written in vector form as

$$
\mathbf{p}(r)=\mathbf{E}(r) \mathbf{T}(r) \mathbf{a}+\mathbf{n}(r),
$$

where $\mathbf{E}$ is a matrix containing the $N$ modeshapes as sampled at the $M$ receiver depths. ${ }^{3}$

$$
\mathbf{E}=\left[\begin{array}{ccc}
\phi_{1}\left(z_{1}, r\right) & \cdots & \phi_{M}\left(z_{1}, r\right) \\
\vdots & \ddots & \vdots \\
\phi_{1}\left(z_{N}, r\right) & \cdots & \phi_{M}\left(z_{N}, r\right)
\end{array}\right]
$$

The range-dependent $R_{i}$ terms may be grouped in an $N \times N$ matrix $\mathrm{T}$, referred to as the propagation matrix. The elements of $\mathbf{T}$ depend on what assumptions are made regarding the propagation of the modes along the path. This is by no means a trivial task, and is the focus of considerable research, particularly after the results of the HIFT. The simplest assumption one can make is the adiabatic assumption, which says that given a slowly varying environment, the modes will propagate without transfer of energy [12]. In other words, there won't be any coupling between the modes. Under this simplifying assumption, the $R_{i}(r)$ are constant, and the off-diagonal terms of $\mathbf{T}$ are zero. The diagonal terms are given by

$$
T_{i, i}=\sqrt{\frac{2}{\pi}} \frac{e^{-j \pi / 4}}{\sqrt{k_{i}(r) r}} e^{j \int_{0}^{r} k_{i}(r) d r} .
$$

One of the main purposes in determining the modal structure at the Monterey VLA is to gain a better understanding as to exactly what assumptions may be made regarding mode coupling.

\footnotetext{
${ }^{3}$ The modeshapes as computed for the Monterey site are shown in Figure 4-1.
} 


\subsection{Objectives}

The propagation from Heard Island to California presents a very complex problem. It has been suggested that " $\ldots$ the $18,000 \mathrm{~km}$ transmission from Heard Island, ... is perhaps the most complicated acoustic propagation problem available," [13]. As a first step in characterizing the propagation, this thesis concentrates on determining the modal content, or vertical structure of the recorded signals. Issues concerning the quality of the datasets are addressed, including low signal levels due to the great distances traveled, inoperable hydrophones due to electrical failures, the lack of accurate array position data due to sensor failures, and also the presence of a Doppler shift due to source movement. Analyses have previously been done on the data by others, including Mikhalevsky at SAIC and Miller at the Naval Postgraduate School (NPS). The primary method of modal analysis developed here is a least squares modal decomposition. The outcome of this work will aid in advancing the general knowledge of long-range, low-frequency acoustic propagation, as well as highlighting areas of concern for future acoustic thermometry work.

\subsection{Organization}

The steps taken in conditioning the data are described in the next chapter. The predicted Doppler shift for each data set is computed and then compared against the measured value, and a preliminary analysis is made of the final processed time sequences. In Chapter 3, the least squares modal beamformer is introduced and its

performance issues are addressed. In Chapter 4, the results of the modal analysis are presented, along with a discussion of how the array orientation was inferred, and comparisons are made with previous, independent analyses of the same data sets. Finally, Chapter 5 contains a summary of the work, the conclusions that can be drawn, and how they may impact future work. 
This page left blank. 


\section{Chapter 2}

\section{Preliminary Processing}

Three different types of signals were developed for the HIFT. All were centered around $57 \mathrm{~Hz}$, which was selected to avoid the 50 and $60 \mathrm{~Hz}$ line noises. The simplest signal was a single $57 \mathrm{~Hz}$ tonal, referred to as the continuous wave (CW) signal. This signal provided the best penetration of low signal-to-noise environments since it concentrated all of its source power into a single band. The other two signal types, pentaline and pseudo-random phase shift, were multi- and broad-band signals

respectively [19]. Because of the low signal-to-noise ratio (SNR) for the Heard Island to Monterey path, only the $\mathrm{CW}$ transmission events will be analyzed.

Of all the $\mathrm{CW}$ transmission events, only data from the first three transmissions were chosen for analysis. During this period, all of the sources were operating and the largest number of hydrophones on the receiving array (21 out of the 32) were functioning. Table 2.1 summarizes the three $\mathrm{CW}$ data sets presented in this thesis. The channels used are the same for all three events. The transmission time is the time at which the signal left Heard Island and the recording time is the time at which data recording commenced at Monterey. This will be time zero for all subsequent data plots. The estimated arrival time is based on the estimated travel time of 3 hours, 19 minutes and 21 seconds [6]. The dataset (or event) naming convention is 
to use the date and approximate time of recording; e.g. 01270322 is the reception event on January 27, at approximately 0322 hours. Note that for the first dataset, the recording was actually started after the transmission arrival.

\begin{tabular}{||c|c|c|c||}
\hline \hline Event & $\begin{array}{c}\text { Transmission Time } \\
\text { (dd/hhmm:ss GMT) }\end{array}$ & $\begin{array}{c}\text { Recording Start } \\
\text { (dd/hhmm:ss GMT) }\end{array}$ & $\begin{array}{c}\text { Estimated Arrival } \\
\text { (dd/hhmm:ss GMT) }\end{array}$ \\
\hline 01261525 & $26 / 1200: 00$ & $26 / 1526: 16$ & $26 / 1519: 21$ \\
01270322 & $27 / 0000: 00$ & $27 / 0300: 54$ & $27 / 0319: 21$ \\
01271505 & $27 / 1200: 00$ & $27 / 1510: 28$ & $27 / 1519: 21$ \\
\hline \hline
\end{tabular}

Table 2.1: Summary of transmission events selected for analysis. All three are CW events.

The signals from the hydrophones were passed through a $10-80 \mathrm{~Hz}$ bandpass filter and then sampled at $228 \mathrm{~Hz}$ before being recorded on optical disks [14]. After the acquisition, several pre-processing, or conditioning, steps were necessary to improve the generally poor data quality. Also, since the transmitted signal was sufficiently narrowband, the carrier frequency could be removed, thereby reducing the sampling rate and saving computation time. Figure 2-1 outlines the data conditioning sequence, which is discussed in the following sections.

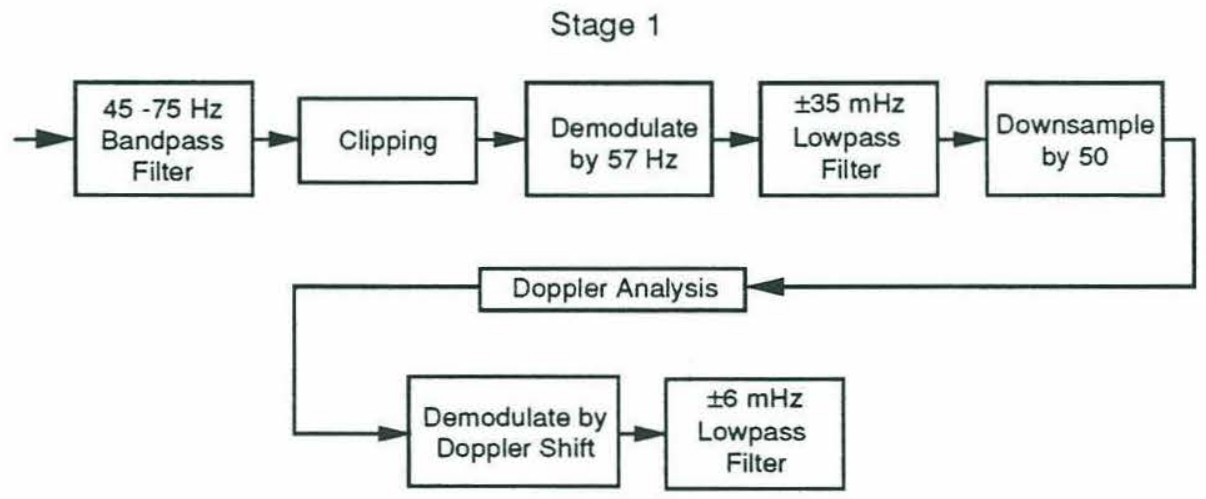

Stage 2

Figure 2-1: Processing flowchart 


\subsection{First Stage}

The goal of this first stage was to condition the signal and reduce the sampling rate so the data sets were of manageable size while being appropriate for the signal bandwidth. A typical event as read off the optical disk required close to 90 Megabytes of memory. Once reduced, each event required only 3 Megabytes or less.

\subsubsection{Spike Removal}

Large transient spikes of up to \pm 5 volts were present throughout the three CW datasets. The suspected cause was a loose hydrophone breakout producing vibrations on the array [7]. Because of the very low signal levels, it was possible to clip the spikes at roughly the background noise level without removing any of the actual signal. Prior to clipping the data, a smooth, 45-75 Hz bandpass filter was applied. This eliminated much of the broadband spike energy that would otherwise be smeared into the signal band during clipping. The impulse response length (101 points, or 0.443 seconds at $228 \mathrm{~Hz}$ sampling) was kept on the same order as the time duration of a spike. Analyses done both with and without the pre-filtering suggest that it did provide a noticeable increase in signal-to-noise levels. Figure 2-2 shows the frequency response for the bandpass filter. After filtering, the data was clipped at a level of one standard deviation. A frequency $v s$ time plot of the data after clipping is shown in Figure 2-3. Note the $60 \mathrm{~Hz}$ line noise and the much heavier noise at $50 \mathrm{~Hz}$, possibly due to shipping. Also note that the $57 \mathrm{~Hz} \mathrm{CW}$ signal is not yet visible above the noise.

\subsubsection{Demodulation and Decimation}

As mentioned earlier, the demodulation/decimation steps were taken to simplify the data manipulation and analysis. The first step was to remove the $57 \mathrm{~Hz}$ carrier 


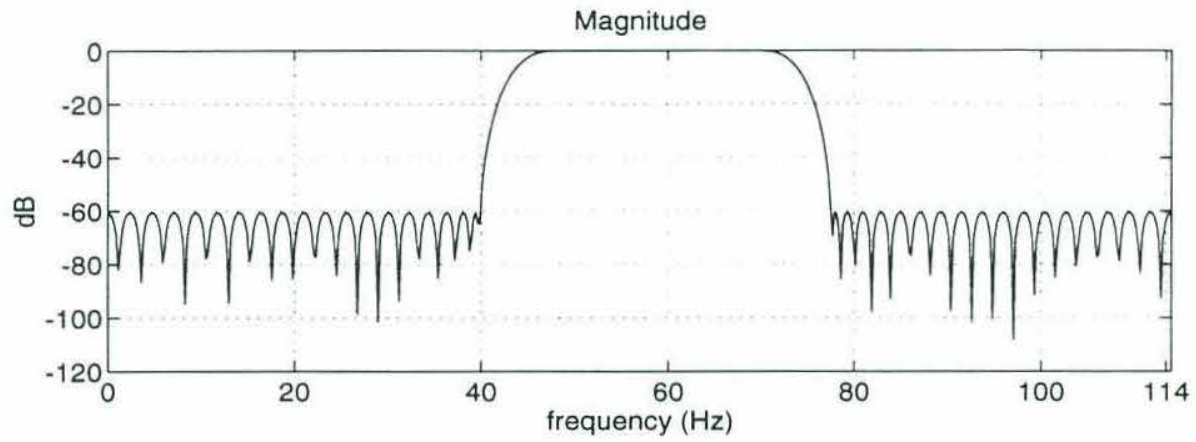

Figure 2-2: Frequency response for the 45-75 Hz, Parks-McClellan design, bandpass filter

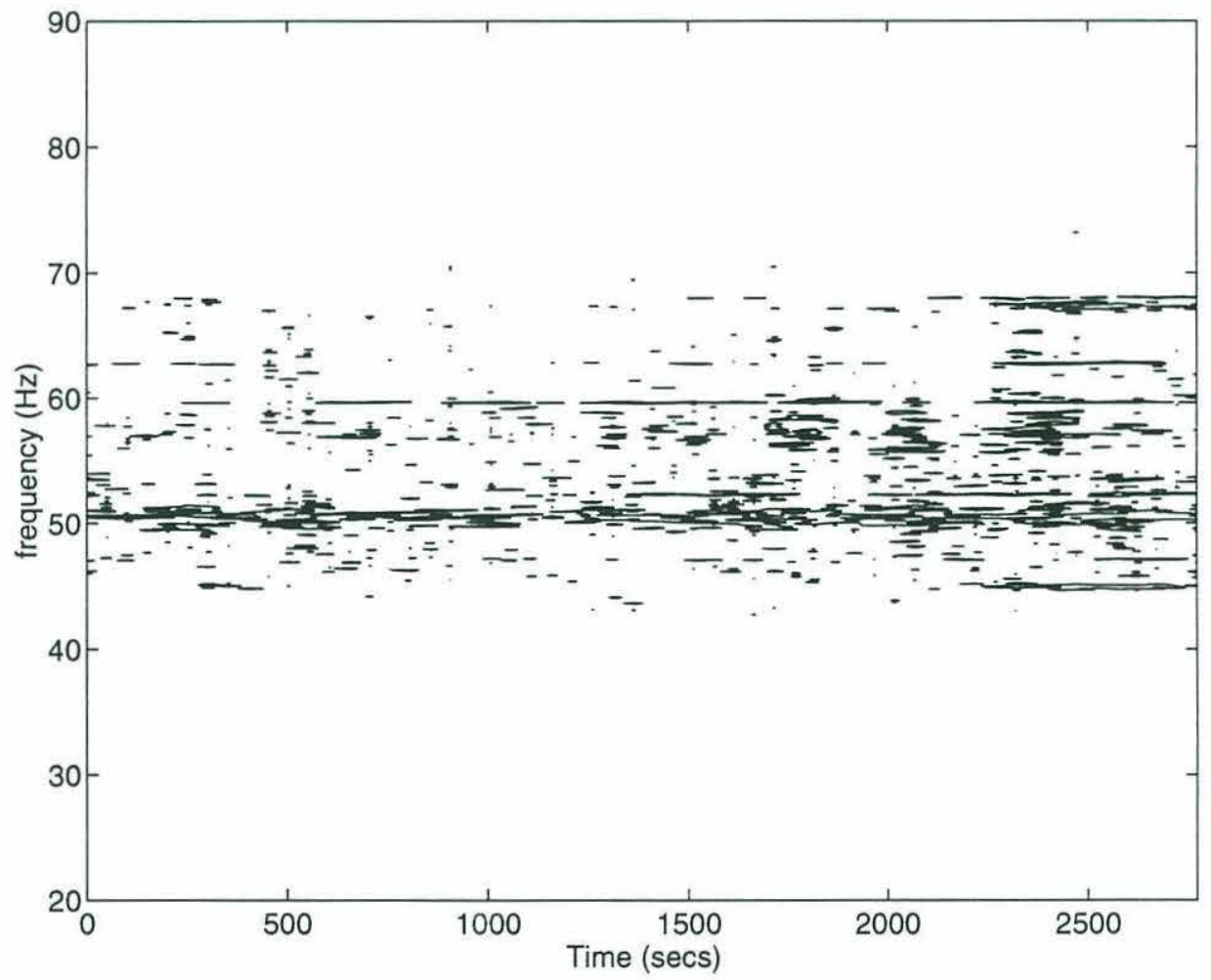

Figure 2-3: Spectogram of 01261525, Channel 0 data after bandpass filtering and clipping. 
frequency by shifting the spectrum down the appropriate number of frequency bins. Since the sampling rate was an even multiple of $57 \mathrm{~Hz}$, the bin shifting resulted in an exact demodulation. The next step was to apply an anti-aliasing filter, designed with a $\pm 35 \mathrm{mHz}$ passband, $150 \mathrm{mHz}$ transition band, and $70 \mathrm{~dB}$ of rejection in the stopband (Figure 2-4). The impulse response length was 10,001 points, or 43.0 seconds at $228 \mathrm{~Hz}$. This yielded a pre-decimation correlation length of about 200 points, or 877 msecs. While a broader filter could have accomplished the necessary anti-aliasing just as easily, it would not have provided as much increase in signal-tonoise ratio. In anticipation of the upcoming section on Doppler shifting, it should be mentioned that a passband of $\pm 35 \mathrm{mHz}$ covers Doppler shifts corresponding to \pm 1.7 knots of boat speed for a $57 \mathrm{~Hz}$ signal launched parallel to the ship's bearing. Alternatively, at a nominal ship speed of 3 knots, this bandwidth corresponds to a relative launch angle variation of $\pm 50^{\circ}$. All $\mathrm{CW}$ transmissions fell well within this range. The last step, the downsampling or decimation, was by a factor of 50 , reducing the sampling rate from $228 \mathrm{~Hz}$ to $4.56 \mathrm{~Hz}$. The data, now in quadrature form, was centered about $0 \mathrm{~Hz}$, plus the Doppler shift, with an effective correlation length of about 4 points.

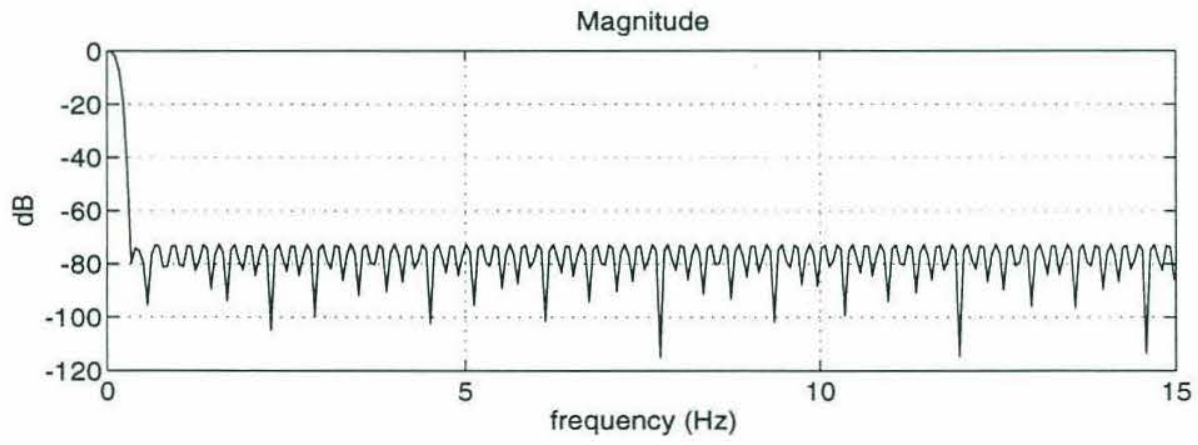

Figure 2-4: Frequency response for the $\pm 35 \mathrm{mHz}$, Parks-McClellan lowpass filter. 


\subsection{Doppler Analysis}

Heavy seas off Heard Island forced the source ship $R / V$ Corey Chouest to maintain constant headway into the wind. This movement of the source introduced a Doppler shift into the transmitted signal. While typically a nuisance and something to be avoided, the addition of a Doppler shift provided an unexpected benefit - it could be used to estimate the launch angles from the source at Heard Island [15], which could then be compared to the launch angles computed by the modeling.

\subsubsection{Prediction}

The expected Doppler shift for each transmission event can be readily predicted from available information. The ship's speed and heading data obtained from GPS readings were exceptionally accurate during this experiment as a result of the Selective Availability feature being turned off for the war in the Persian Gulf. The horizontal ray path the signal followed from source to receiver was determined from modeling by Chiu, et al. prior to the experiment [6]. From this, the launch angle at the source can be estimated. The predicted azimuth leaving Heard Island was between 133 and 136 degrees (measured clockwise from the north). The Doppler shift for a moving source with stationary receiver and medium is given by

$$
f_{\text {dop }}=f_{o} \frac{U}{C} \cos \left(\alpha_{s}-\alpha\right),
$$

where

$$
\begin{aligned}
f_{\text {dop }} & =\text { resulting Doppler shift in received signal, } \\
f_{0} & =57 \mathrm{~Hz} \text { carrier frequency, } \\
U & =\text { ship's speed, } \\
C & =\text { sound speed at source (on SOFAR axis) } 1455 \mathrm{~m} / \mathrm{s}, \\
\alpha_{s} & =\text { ship's bearing, and } \\
\alpha & =\text { signal launch angle. }
\end{aligned}
$$


Substituting in the information from the first three columns of Table 2.1.2 yields predicted frequency shifts shown in the Predicted Doppler column of the same table. The calculations were done for both sides of the estimated launch envelope, 133 and 136 degrees, giving a range of possible Doppler shifts. Note that all shifts are negative, indicating the source is moving away from the direction of propagation. It should be pointed out that there is a differential Doppler shift associated with rays leaving at different vertical angles from the source, implying then that each mode would have a slightly different shift. These shifts, however, are much smaller than the above Doppler shifts, and are therefore ignored [16].

\begin{tabular}{||c|c|c|c|c|c|c||}
\hline \hline Data set & $\begin{array}{c}\text { Bearing } \\
(\mathrm{deg})\end{array}$ & $\begin{array}{c}\text { Speed } \\
(\mathrm{kts})\end{array}$ & $\begin{array}{c}\text { Launch } \\
\text { Angle } \\
(\mathrm{deg})\end{array}$ & $\begin{array}{c}\text { Predicted } \\
\text { Doppler } \\
(\mathrm{mHz})\end{array}$ & $\begin{array}{c}\text { Measured } \\
\text { Doppler } \\
(\mathrm{mHz})\end{array}$ & $\begin{array}{c}\text { Estimated } \\
\text { Launch Angle } \\
(\mathrm{deg})\end{array}$ \\
\hline 01261525 & 254.5 & 2.99 & $133-136$ & $-31.5--28.7$ & -30.5 & 134.1 \\
01270322 & 252.0 & 2.51 & $133-136$ & $-24.5--22.2$ & -24.0 & 133.7 \\
01271505 & 234.5 & 3.21 & $133-136$ & $-12.6--9.6$ & -12.5 & 133.4 \\
\hline \hline
\end{tabular}

Table 2.2: Comparison of predicted and measured Doppler shift for the CW signals received on the VLA.

\subsubsection{Measurement}

As seen in the table, the predicted Doppler shifts were on the order of $10-30 \mathrm{mHz}$. A shift in the measured Doppler of just $\pm 1 \mathrm{mHz}$ at the array could result in about a 3 degree shift in the estimation of the launch angle. Because of this sensitivity, accuracy on the order of $\pm 1 \mathrm{mHz}$ was desired, thus dictating an FFT with a length of at least 1000 seconds. The data was windowed using a 1000-second Hanning window, with half-window advances between FFT's, essentially making each window an independent sample. Two-dimensional frequency vs time plots were created, from which the actual Doppler shift was read. Figures 2-5 - 2-7 show examples of the Doppler shifts as seen for each of the $3 \mathrm{CW}$ datasets. Recall that because of the 
demodulation, the signal should be centered around $0 \mathrm{~Hz}$. In the 01270322 sonogram, the energy scattered above and below the signal frequency is due possibly to the ship pulling on the array.

The results for the three transmission events are summarized in the last two columns of Table 2.1.2. There was remarkable agreement between the predicted and observed shifts. This proved an effective means of verifying that the signals received at Monterey did indeed follow the path predicted prior to the experiment. Equation 2.1 can be inverted to solve for the launch angle using the measured Doppler. As indicated, the estimated launches are within one degree of each other, as well as within the predicted launch window.
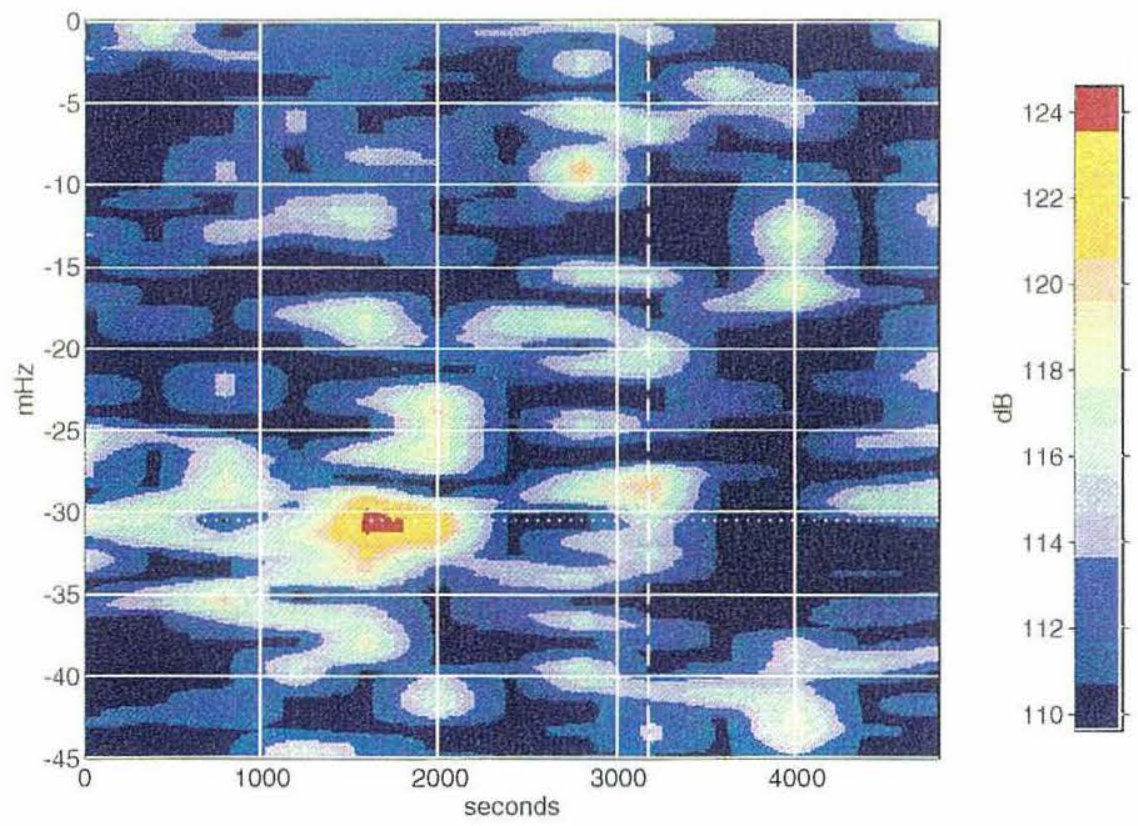

Figure 2-5: Sonogram - Channel 0, Event 01261525. Horizontal dotted line is estimated Doppler shift and vertical dashed lines indicate predicted signal duration. 


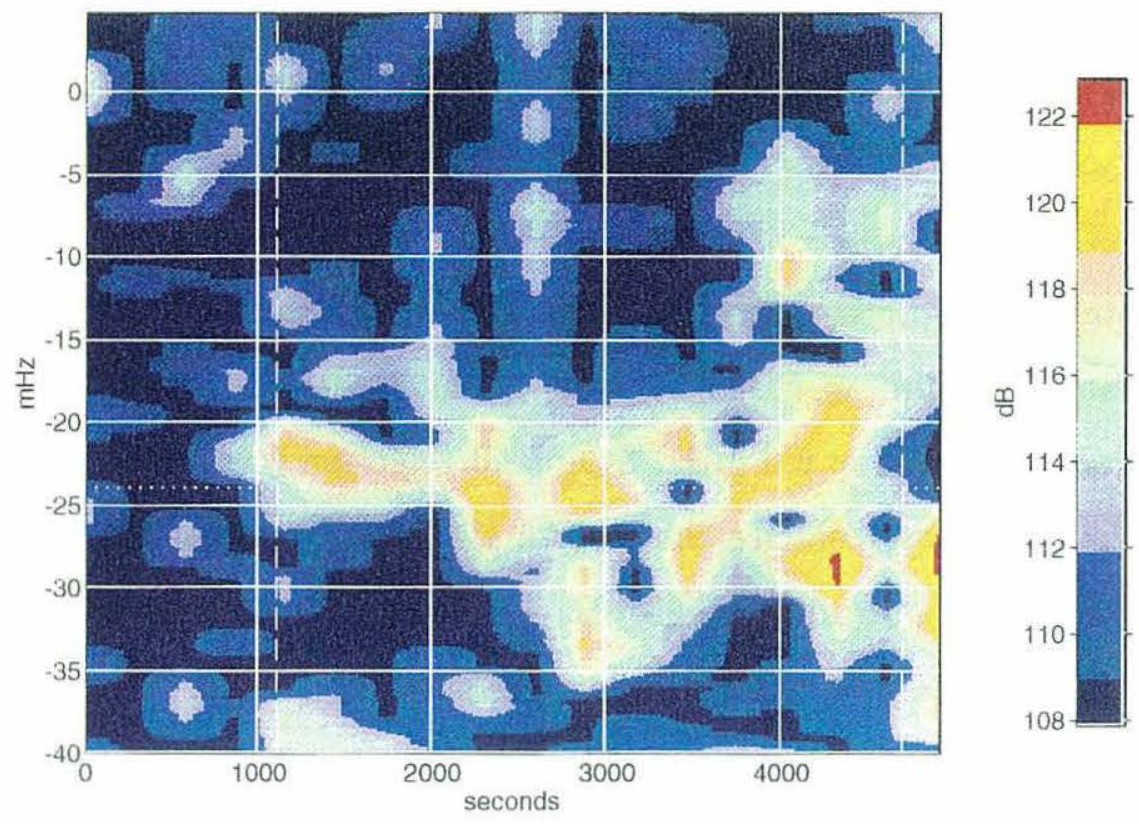

Figure 2-6: Sonogram - Channel 0, Event 01270322. Horizontal dotted line is estimated Doppler shift and vertical dashed lines indicate predicted signal duration.
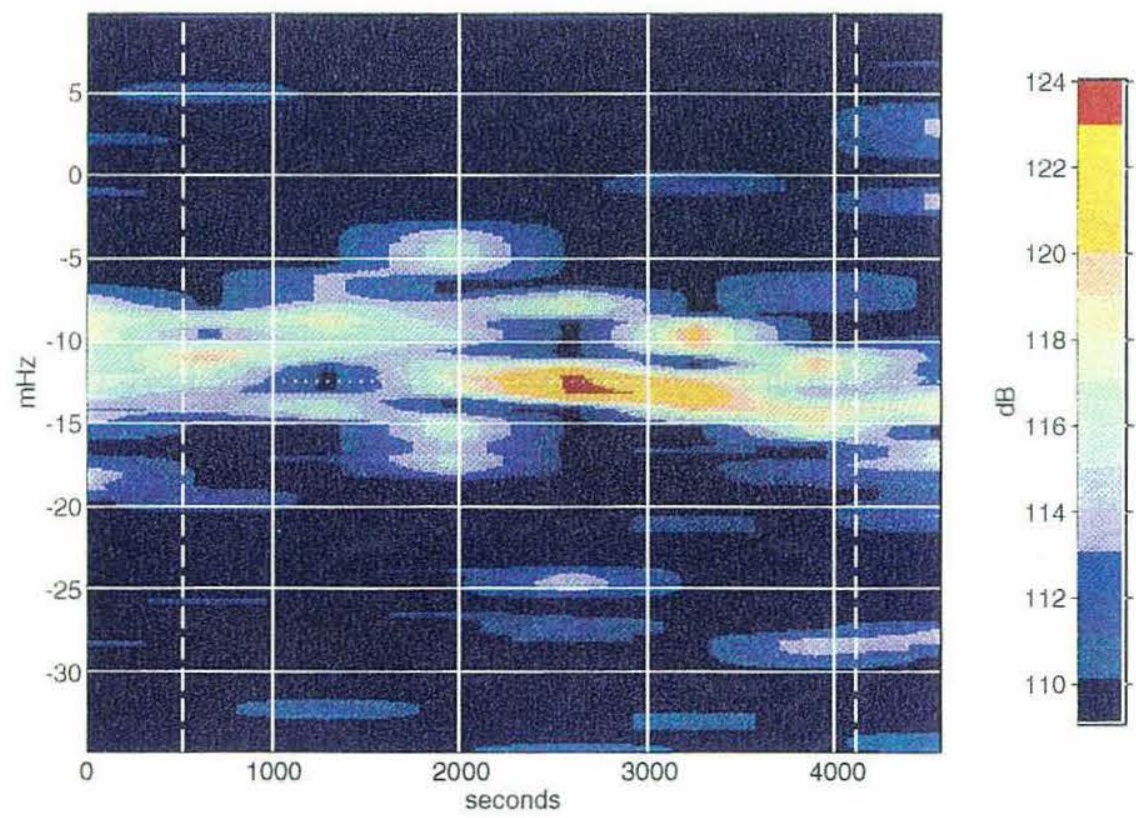

Figure 2-7: Sonogram - Channel 0, Event 01271505. Horizontal dotted line is estimated Doppler shift and vertical dashed lines indicate predicted signal duration. 


\subsection{Second Stage}

With the Doppler shift now established for each dataset, the next step was to remove it by a second demodulation, placing the signal at baseband. Knowing the signal was exactly at $0 \mathrm{~Hz}$ made it possible to then apply a very narrowband lowpass filter in an attempt to improve upon the low input signal-to-noise ratios. Using a ParksMcClellan algorithm, an FIR filter was designed with a passband of only $\pm 5 \mathrm{mHz}$, and a transition band of $6.0 \mathrm{mHz}$. Stopband rejection was around $70 \mathrm{~dB}$. Figure 2-8 shows the frequency response out to $0.25 \mathrm{~Hz}$. As a result of the filtering, the SNR was increased by a substantial $25 \mathrm{~dB}$. The cost of this, however, was that such a narrow filter required integration lengths of over 600 seconds, resulting in significant time-smearing of the data. The effective filter length, or correlation length, was about 200 seconds.

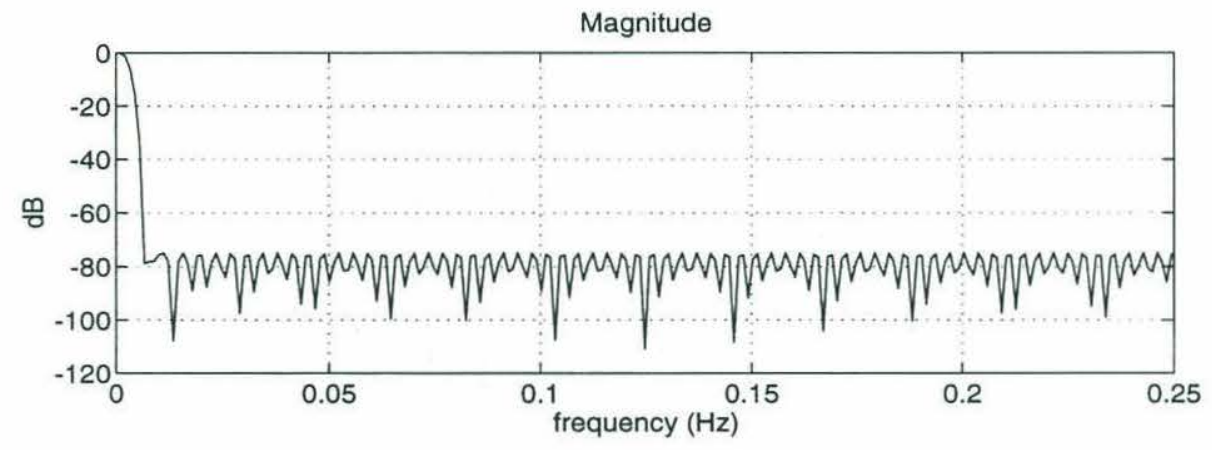

Figure 2-8: Frequency response for the $\pm 5 \mathrm{mHz}$ lowpass filter. 


\subsection{Preliminary Analysis}

With the signal conditioning completed, a preliminary analysis of the time series from each channel was made. Magnitude and phase plots for the three data sets are shown in Figures 2-9 - 2-11. Note that the magnitude levels have not been corrected for any gain due to processing nor for hydrophone sensitivity. The vertical lines in the figures indicate the approximate starting times of the received signal, as computed using the predicted travel time of $3 \mathrm{hrs}, 19 \mathrm{~min}, 21$ secs. Again, the signal arrived prior to the start of the 1525 recording. Looking at the start of the 0322 and 1505 data sets, what appears to be the transmission arrival can be seen relatively close to the expected arrival time, particularly in the magnitude data. The ending of the transmission is less clear, likely obscured by motion of the array. By the end of each transmission hour, all of the slack in the array cable was taken up by the drifting ship, which would then start pulling on the array. This was certainly the case for the 0322 data set. During and after event 1505, the array was particularly stable, and the end of the transmission is apparent about an hour after the signal arrival. The most striking feature in the data is the constant fading in and out of the magnitude, on time scales ranging from 100 to 1000 seconds. Potential explanations for this include a complex, time-varying, interference pattern set up by the arriving modes, or oceanic processes such as internal waves or meso- or gyre-scale eddies. With only three datasets available, it is difficult to obtain more insight into this issue.

Consider for a moment the effect on the signal phase if the Doppler shift was not accurately estimated and/or varied with time. Since the signal is at $0 \mathrm{~Hz}$, residual

Doppler shifting would appear as gradual sloping of the phase. A positive slope would indicate the Doppler was under-estimated and that the phase is advancing with time. A negative slope would indicate the opposite. There are no such trends readily apparent across all the channels for any of the datasets, although channels 
11-14 of Event 1505 do exhibit a fairly uniform phase shift, increasing over the period 500 to 2000 seconds, then decreasing for the next 1500 seconds. This corresponds to a three-quarters of a cycle change over 1500 seconds, or about $0.5 \mathrm{mHz}$, which is within the error margin of the Doppler estimate. The array was quite steady throughout this time period, so the most likely source of this shifting is slight changes in course and speed of the ship. The appearance of this shift on only some channels is puzzling, but may simply be a result of the low signal levels.

Estimates of the actual signal pressure levels at the array can be made by adjusting the conditioned data to account for hydrophone sensitivity, gain through the data acquisition system, and the processing gain. The effective sensitivity through all of the hardware (hydrophones, pre-amps, and other data acquisition equipment) is known to be $-150 \mathrm{~dB}$ re $1 \mathrm{~V} / \mu \mathrm{Pa}$ [17]. The gain through all of the pre-processing stages was estimated by passing a $57 \mathrm{~Hz}$, unit-amplitude sinusoid through each stage and measuring the rms amplitude at the output. Effective pre-processing gain was found to be $-3 \mathrm{~dB}$, including a $6 \mathrm{~dB}$ addition to convert from one-sided (complex envelope) back to two-sided representation. The estimated rms values in the water at the array are shown in the third column of Tables $2.3-2.5$ for the first 14 channels. These levels are similar those seen by G. Heard with the COAMS array [18]. Noise measurements taken at Monterey throughout the course of the experiment show an average noise level of $89 \mathrm{~dB}$ re $1 \mu \mathrm{Pa} / \sqrt{\mathrm{Hz}}$. Over a $1 \mathrm{~Hz}$ band, then, the singlechannel SNR is about $-15 \mathrm{~dB}$. Estimates of the total CW transmission loss may be found by subtracting the signal levels from the source level, as shown in fifth column of the tables.

In one final look at the time series data, the total power across the top fourteen channels is plotted in Figure 2-12 for the three transmissions. The transmission is quite clear for the 01271505 event, and the signal is about $12 \mathrm{~dB}$ higher than the background noise. 


\begin{tabular}{||c|c|c|c|c||}
\hline \hline Channel & $\begin{array}{c}\text { Depth } \\
\text { meters }\end{array}$ & $\begin{array}{c}\text { RMS pressure } \\
d B \text { re } 1 \mu P a\end{array}$ & $\begin{array}{c}\text { Variance } \\
\mathrm{dB}\end{array}$ & $\begin{array}{c}\text { Transmission Loss } \\
\mathrm{dB}\end{array}$ \\
\hline 1 & 345 & 78.0 & 68.4 & 143.1 \\
2 & 390 & 77.4 & 67.3 & 143.7 \\
3 & 435 & 78.3 & 68.6 & 142.8 \\
4 & 480 & 77.0 & 68.5 & 144.1 \\
5 & 525 & 76.5 & 67.3 & 144.6 \\
6 & 570 & 77.5 & 67.2 & 143.6 \\
7 & 615 & 75.4 & 65.3 & 145.7 \\
8 & 660 & 76.4 & 67.2 & 144.7 \\
9 & 705 & 75.9 & 63.7 & 145.2 \\
10 & 750 & 74.3 & 63.8 & 146.8 \\
11 & 795 & 74.1 & 65.3 & 147.0 \\
12 & 840 & 74.8 & 65.2 & 146.3 \\
13 & 885 & 77.0 & 67.1 & 144.1 \\
14 & 930 & 77.3 & 66.0 & 143.8 \\
\hline Average & \multicolumn{3}{|c|}{} \\
\hline \hline
\end{tabular}

Table 2.3: Signal statistics for Event 01261525 for the time period 0 - 2500 seconds. Source level: $221.1 d B$.

\begin{tabular}{||c|c|c|c|c||}
\hline \hline Channel & $\begin{array}{c}\text { Depth } \\
\text { meters }\end{array}$ & $\begin{array}{c}\text { RMS pressure } \\
d B \text { re } 1 \mu P a\end{array}$ & $\begin{array}{c}\text { Variance } \\
\mathrm{dB}\end{array}$ & $\begin{array}{c}\text { Transmission Loss } \\
\mathrm{dB}\end{array}$ \\
\hline 1 & 345 & 77.2 & 64.9 & 143.8 \\
2 & 390 & 79.5 & 69.5 & 141.5 \\
3 & 435 & 80.0 & 70.4 & 141.0 \\
4 & 480 & 76.3 & 65.1 & 144.7 \\
5 & 525 & 78.2 & 68.5 & 142.8 \\
6 & 570 & 77.2 & 67.7 & 143.8 \\
7 & 615 & 77.6 & 67.6 & 143.4 \\
8 & 660 & 78.6 & 70.5 & 142.4 \\
9 & 705 & 78.0 & 69.1 & 143.0 \\
10 & 750 & 77.0 & 67.1 & 144.0 \\
11 & 795 & 76.1 & 66.8 & 144.9 \\
12 & 840 & 78.1 & 69.9 & 142.9 \\
13 & 885 & 79.0 & 69.7 & 142.0 \\
14 & 930 & 78.1 & 69.3 & 142.9 \\
\hline Average & \multicolumn{3}{|l}{} \\
\hline \hline
\end{tabular}

Table 2.4: Signal statistics for Event 01270322 for the time period $1000-3000$ seconds. Source level: $221.0 d B$. 


\begin{tabular}{||c|c|c|c|c||}
\hline \hline Channel & $\begin{array}{c}\text { Depth } \\
\text { meters }\end{array}$ & $\begin{array}{c}\text { RMS pressure } \\
d B \text { re } 1 \mu P a\end{array}$ & $\begin{array}{c}\text { Variance } \\
\mathrm{dB}\end{array}$ & $\begin{array}{c}\text { Transmission Loss } \\
\mathrm{dB}\end{array}$ \\
\hline 1 & 345 & 77.6 & 65.7 & 142.2 \\
2 & 390 & 76.5 & 67.0 & 143.3 \\
3 & 435 & 76.6 & 65.8 & 143.2 \\
4 & 480 & 77.1 & 67.2 & 142.7 \\
5 & 525 & 77.8 & 68.7 & 142.0 \\
6 & 570 & 78.8 & 69.8 & 141.0 \\
7 & 615 & 79.7 & 68.2 & 140.1 \\
8 & 660 & 79.9 & 69.7 & 139.9 \\
9 & 705 & 76.7 & 67.9 & 143.1 \\
10 & 750 & 77.4 & 67.2 & 142.4 \\
11 & 795 & 78.8 & 70.8 & 141.0 \\
12 & 840 & 76.8 & 67.5 & 143.0 \\
13 & 885 & 77.8 & 68.1 & 142.0 \\
14 & 930 & 78.5 & 70.0 & 141.3 \\
\hline Average & \multicolumn{3}{|l||}{} \\
\hline \hline
\end{tabular}

Table 2.5: Signal statistics for Event 01271505 for the time period $1000-3000$ seconds. Source level: $219.8 d B$. 


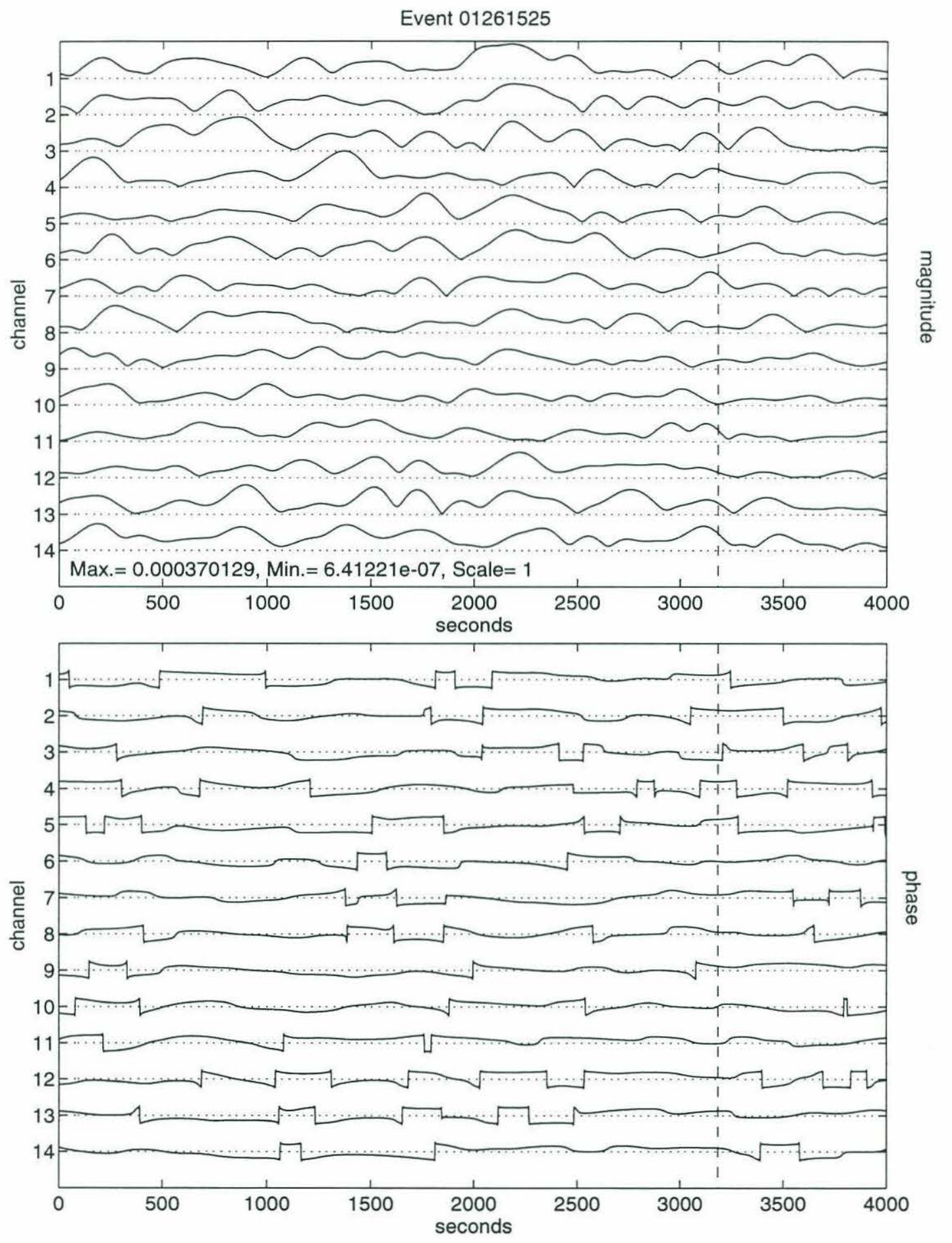

Figure 2-9: Magnitude and phase for Event 01271525. 
Event 01270322
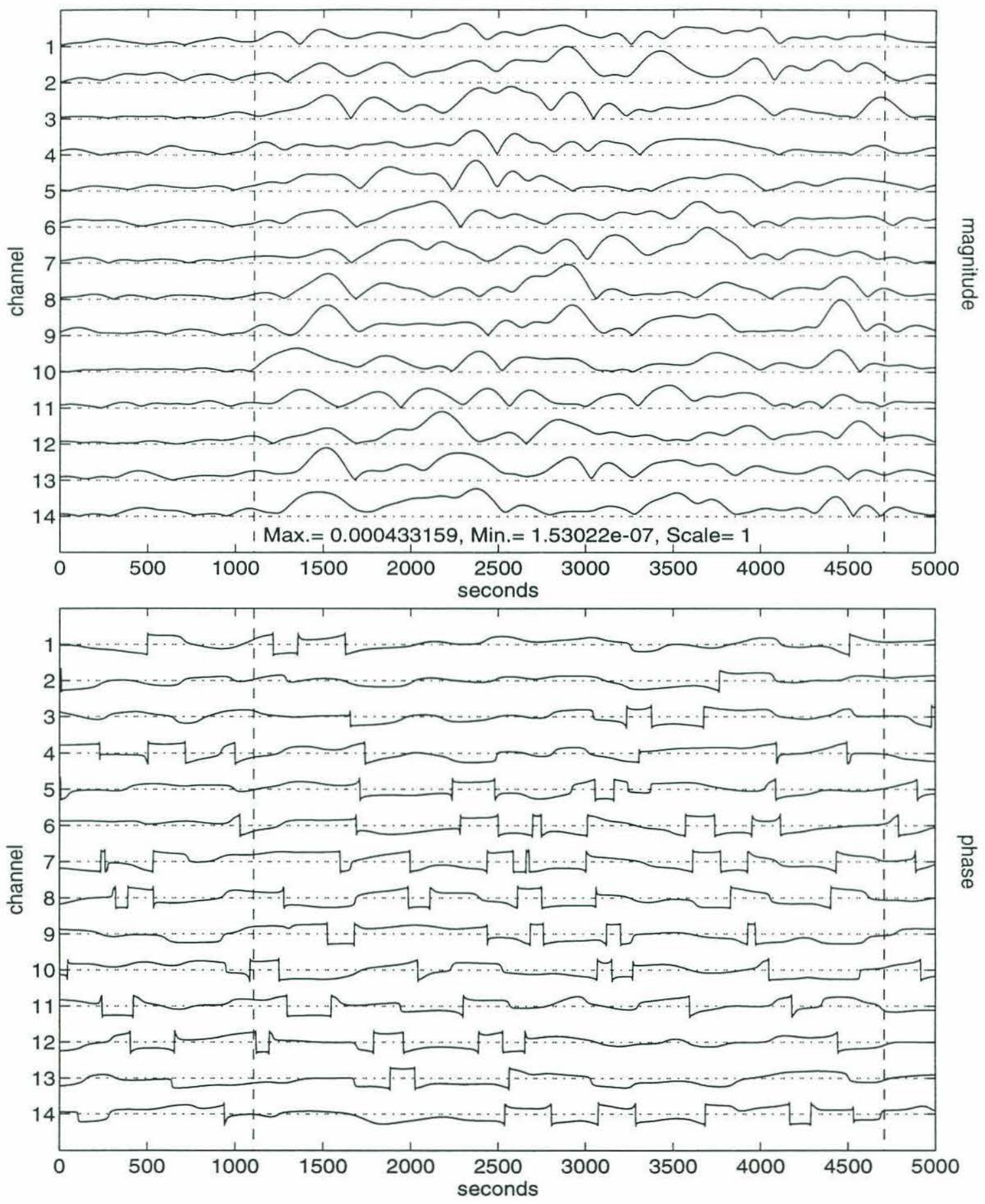

Figure 2-10: Magnitude and phase for Event 01270322. 
Event 01271505
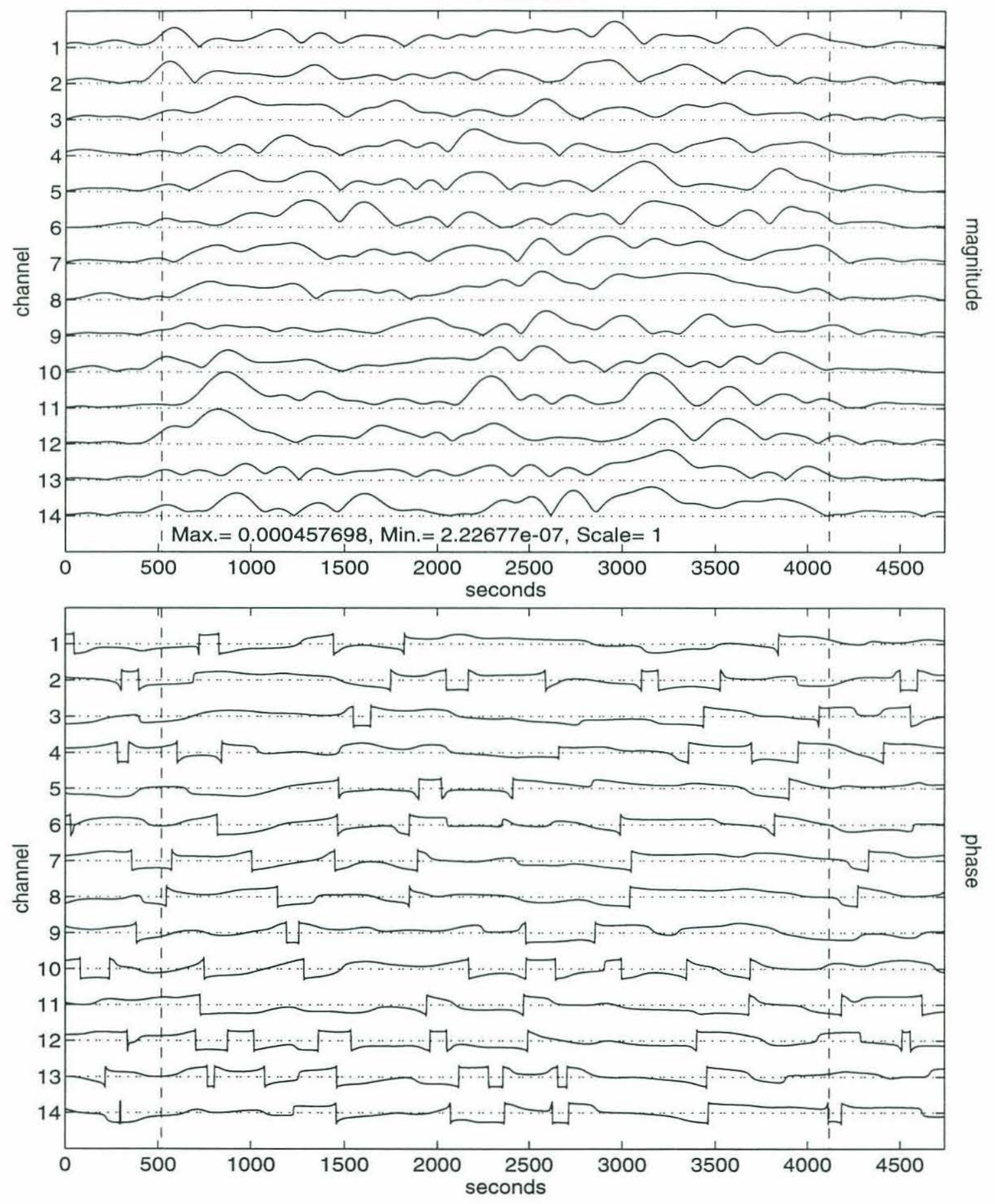

Figure 2-11: Magnitude and phase for Event 01271505. 

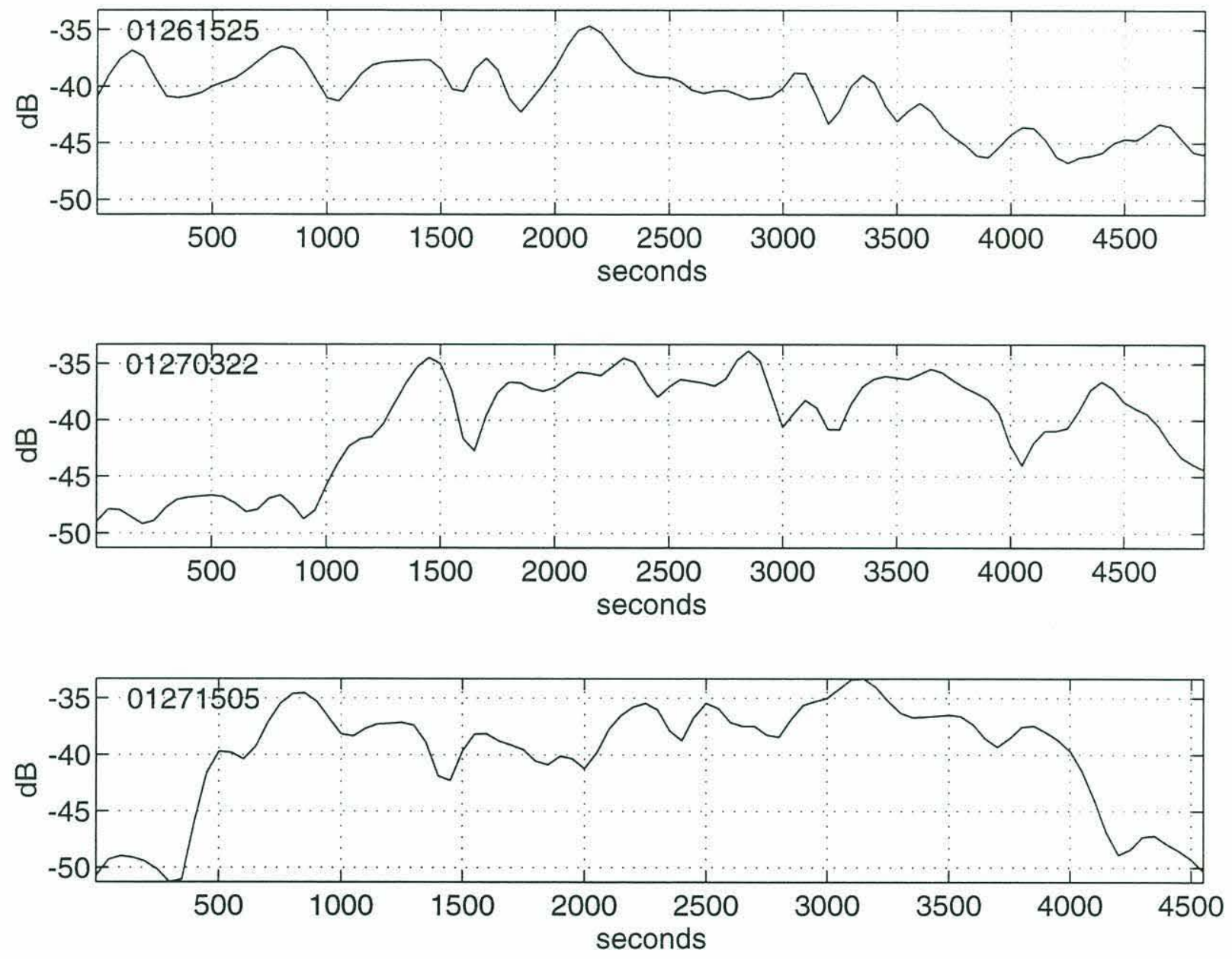

Figure 2-12: Total power across the array for the three CW transmissions. 


\section{Chapter 3}

\section{Least Squares Modal Beamforming}

The last section of the previous chapter focused on some of the time-domain characteristics of the vertical line array (VLA) data. While this yielded useful information, there is much more information to be obtained by looking at the spatial structure of the signal. Indeed, the main reason for deploying a vertical array in the Heard Island experiment was to resolve the low-order mode arrivals, or near-axial rays, something not possible in the time domain. The environmental motivation for analyzing the modal content of the signal was discussed in the introduction.

There are several methods of analyzing the modal structure of the receptions [20]. Because of its relative ease of implementation, as well as robustness, the method used here is a modal fitting of the data based on linear least squares theory. An alternative method of analyzing the vertical structure is via beamforming. There is, however, a very close relationship between the least squares fitting and beamforming. As a result, the least squares algorithm will often be referred to as the least squares modal beamformer.

The simplest implementation of a modal beamformer is the single-mode beam- 
former, where the received signal is projected onto the mode space. This can be considered the spatial analog to the matched filter of the time domain. Since the modeshapes are frequency-dependent the data must be transformed to the frequency domain and the desired frequency bin extracted. Equation (1.1) in Chapter 1 showed how the pressure field field could be represented as a sum of normal modes. If the excitation and range coefficients are combined, the new coefficient can be estimated as shown below for mode $i$ :

$$
\hat{a}_{i}=\frac{\phi_{i}^{+}}{\left\|\phi_{i}\right\|^{2}} \mathbf{p},
$$

where the + superscript indicates the conjugate transpose of the vector. It is easy to see that if the received field consists only of a single mode $i, \mathbf{p}=\alpha \boldsymbol{\phi}_{i}$, then the estimation is exact, and $\hat{a}_{i}=\alpha$. This method for determining the mode coefficients works well in situations where the sensors are closely spaced and the array spans at least enough of the water column so that all propagating modes are within the array aperture. These two conditions are necessary to avoid spatial aliasing of the sampled modeshapes. When these conditions are not met, the modeshapes are no longer orthogonal to one another. As a result when (3.1) is used, there is modal crosstalk, or energy leaking from one mode into the estimate of another. The amount leaking from mode $i$ to $j$ is proportional to the correlation between the two modeshapes. Such crosstalk can falsely indicate the presence of modes which are not there. One way of displaying the crosstalk is by plotting the modal covariance matrix. The larger the off-diagonal values, the greater the coupling between the modes. Figure 3-1 illustrates the coupling for the instance where only the top 14 sensors of the Monterey VLA are used. One method of eliminating this problem is to use the least squares approach, where the signal is modeled as a linear combination of $M$ modes and the associated coefficients are those that minimize the mean square error.

In the following section, the least squares algorithm, or beamformer, is discussed and contrasted with the single-mode beamformer. A modification to the least squares 

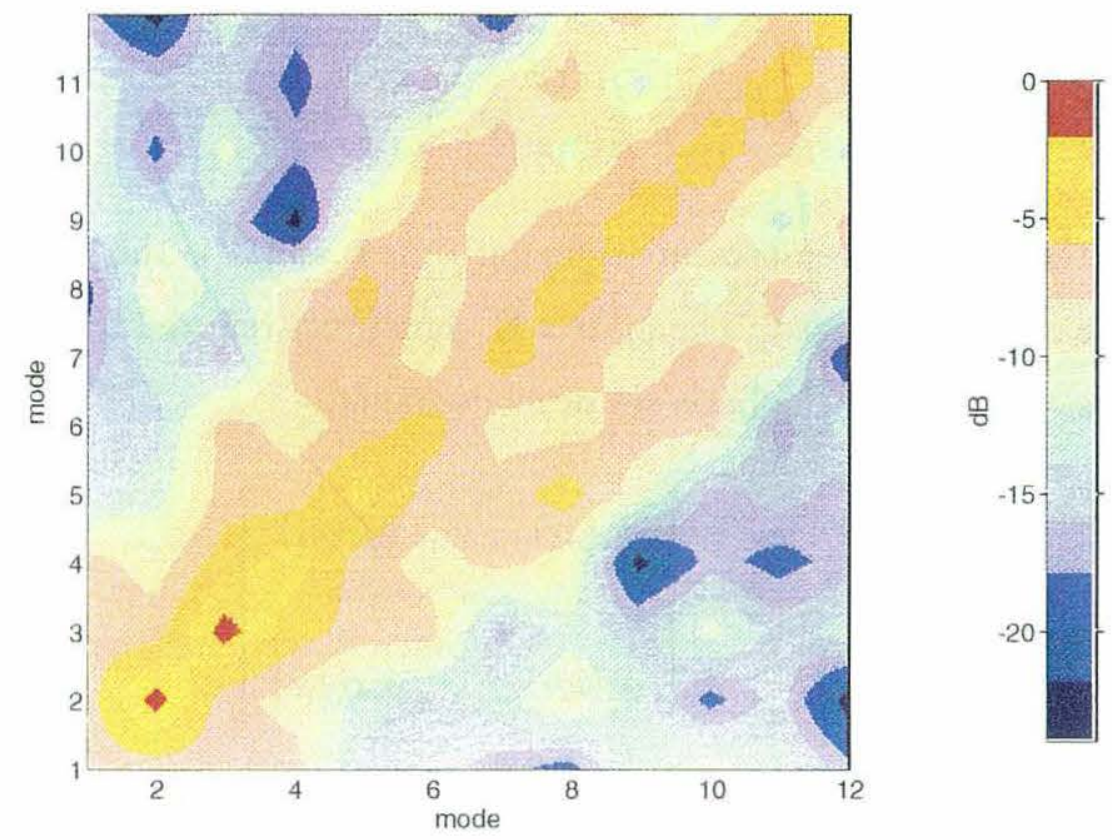

Figure 3-1: Covariance matrix for modes 1-12, computed using only the top 14 sensors

beamformer is introduced to compensate for loss of mode orthogonality due to the reduced array aperture. The issue of how many modes to include in the basis set is addressed, and the chapter closes with an evaluation of the beamformer's performance versus signal-to-noise ratios and variations in array tilt and azimuth. All of the numerical results in this chapter were computed using synthetic data from the first 14 channels of the array. This allows the synthetic results to be related more directly to the results from the real data, where the analysis is similarly constrained.

\subsection{Formulation}

The least squares algorithm derives its name from the process of determining the parameter values that minimize the mean square estimation error. The parameters of interest here are the estimated mode amplitudes, expressed in vector form as 
$\hat{\mathbf{a}}(t)=\left[\hat{a}_{1}(t) \cdots \hat{a}_{M}(t)\right]$. The estimation error $\mathcal{E}$ at time $t$ is given by

$$
\mathcal{E}(t)=\frac{1}{T} \int_{t-T / 2}^{t+T / 2}|\mathbf{p}(\tau)-\mathbf{E} \hat{\mathbf{a}}(t)|^{2} d \tau
$$

where $\mathbf{p}(t)$ is the received pressure signal at time $t$, and $T$ is the window length over which the error is averaged. The matrix $\mathbf{E}$, sometimes referred to as the observation matrix, is composed of the basis set of modeshapes.

$$
\mathbf{E}=\left[\begin{array}{ccc}
\phi_{1}\left(z_{1}\right) & \cdots & \phi_{M}\left(z_{1}\right) \\
\vdots & \ddots & \vdots \\
\phi_{1}\left(z_{N}\right) & \cdots & \phi_{M}\left(z_{N}\right)
\end{array}\right]
$$

If the amplitude of mode $i$ is to be estimated, that mode must be included in the basis set. The $\phi_{i}$ are the tilt-corrected (and therefore complex) modeshapes. Because the modeshapes are frequency-dependent, it is more convenient to do this in the frequency domain, and so $T$ in (3.2) becomes the FFT window length. The estimated coefficients are found using the standard linear least squares formula, found in numerous references [22, 23].

$$
\hat{\mathbf{a}}(t)=\underset{\hat{\mathbf{a}}}{\operatorname{argmin}} \mathcal{E}(t)=\left(\mathbf{E}^{+} \mathbf{E}\right)^{-1} \mathbf{E}^{+} \mathbf{p}(t)
$$

The inverse in the above expression for $\hat{\mathbf{a}}(t)$ is computed using a complex singularvalue decomposition.

As with the single-mode beamformer, if there is no noise present and the received field is a linear combination of normal modes, where each mode is also included in the signal representation, (3.4) will yield the exact coefficients, $\boldsymbol{\alpha}$, and the residual 
error will be zero.

$$
\begin{aligned}
& \mathrm{p}=\mathbf{E} \boldsymbol{\alpha} \\
& \hat{\mathrm{p}}=\mathbf{E} \hat{\mathbf{a}}=\mathbf{E}\left(\mathbf{E}^{+} \mathbf{E}\right)^{-1} \mathbf{E}^{+} \mathbf{p}=\mathbf{E} \boldsymbol{\alpha} \\
& \mathcal{E}=E\left[|\mathbf{p}-\hat{\mathbf{p}}|^{2}\right]=E\left[|\mathbf{E} \boldsymbol{\alpha}-\mathbf{E} \boldsymbol{\alpha}|^{2}\right]=0
\end{aligned}
$$

The mean square error of (3.2) may be used as a measure of how well the signal $\mathbf{p}(t)$ is being estimated. Ignoring the time-dependence, this may be computed as

$$
\mathcal{E}=E\left[|\mathbf{p}-\hat{\mathbf{p}}|^{2}\right]=2 E\left[\mathbf{p}^{+} \mathbf{p}\right]-2 E\left[\hat{\mathbf{p}}^{+} \mathbf{p}\right]=2 \operatorname{tr}\left(\mathbf{R}_{\mathbf{p}}\right)-2 \operatorname{tr}\left(\mathbf{R}_{\hat{\mathbf{p}} \mathbf{p}}\right)
$$

Here, $\mathbf{R}_{\mathbf{p}}$ and $\mathbf{R}_{\hat{\mathrm{p} p}}$ are the correlation and cross-correlation matrices, respectively, and $\operatorname{tr}()$ indicates the trace of the matrix. The error can be normalized by the total signal power, $\operatorname{tr}\left(\mathbf{R}_{\mathbf{p}}\right)$, and subtracted from unity to yield a measure of the how well the estimate fits the observed data. The fit, denoted by $\eta$, is defined as:

$$
\eta=\frac{\operatorname{tr}\left(\mathbf{R}_{\hat{\mathbf{p}} \mathbf{p}}\right)}{\operatorname{tr}\left(\mathbf{R}_{\mathbf{p}}\right)} .
$$

In the processing, the modeshapes that form the basis set are evaluated at each sensor location on the array, and so become a function of the array orientation. Therefore the fit, $\eta$, may also be considered a function of the array orientation, i.e. tilt and azimuth (relative to direction of signal propagation). This idea will be used later when trying to infer array orientation from the acoustic data.

Insight may be gained into the behavior of the least squares beamformer by noting its similarity to an adaptive beamformer. The single-mode beamformer of Equation 3.1 can be considered the conventional modal beamformer. It is steered towards mode $i$ and there is leakage from nearby modes. The adaptive solution to this interference is to place nulls in the beampattern in the direction of the adjacent 
modes that are interfering. The least squares beamformer does essentially the same thing, placing nulls in the modal beampattern by subtracting from the single-mode estimated value any contributions that may be due to leakage from other modes.

The modal beampatterns of the two different beamformers can be found by "steering" each beamformer towards a particular mode and plotting the response versus a range of inputs, where the inputs are taken to be individual modes. Steering to mode $i$ with the single-mode beamformer simply requires substituting the $i^{\text {th }}$ modeshape into Equation 3.1. With the least squares, the response at mode $i$ is given by the $i^{\text {th }}$ coefficient in $\hat{\mathbf{a}}$, provided mode $i$ is one of the basis modeshapes. When the steering mode and input mode coincide, the response is unity. That is, there is a unity gain constraint on the main response axis. Figure 3-2 shows the beampatterns for seven different steering modes. The least squares response was computed using the first seven modes as a basis. Both beamformers were evaluated using only the first 14 channels on the array. Note the leakage from adjacent modes for the single-mode beamformer, and the very deep nulls placed over those same adjacent modes by the least squares. Also note that for modes 8 and higher, the least squares has a higher response than the single-beam. This increased response to higher modes creates two problems. First, the presence of even a small amount of any mode not included in the observation matrix can dominate estimates of low-order mode amplitudes. This can be generally be avoided by carefully choosing $M$, the number of modes in $\mathbf{E}$, to include all modes possibly present in the water column. ${ }^{1}$ To understand the second problem, notice that the level of higher mode leakage depends on the steering mode. For example, when steered towards mode 1, the leakage is lower compared to that when steered towards mode 5. This introduces what amounts to a bias among the mode estimates. A measure of this bias can be obtained by comparing the beamformer output power at various steering modes. For an input signal, assume the

\footnotetext{
${ }^{1}$ The number of modes $M$ cannot be greater than $N$, the number of sensors on the array.
} 
uniform excitation of the first 16 modes. Ideally, since the power in each input mode is the same, the response power at each steering mode should be also be the same. Figure 3-3 shows that indeed this is nearly true if the least squares is used with all 32 array elements. With only 14 elements, however, the orthogonality between the modeshapes is destroyed and a large bias results. Mode 5 is estimated to be almost $30 \mathrm{~dB}$ higher than mode 1 . The single-mode case with equal excitation of all modes is included for comparison. Its response is much flatter, with only about a $5 \mathrm{~dB}$ range in total response power.

While the leakage in the single-mode case is greater than in the least squares case, the reduced orthogonality makes the least squares bias much worse than the singlemode. This can be seen if the received signal is modeled as a linear combination of normal modes plus contributions from noise.

$$
\mathrm{p}=\mathbf{E} \boldsymbol{\alpha}+\mathrm{n}
$$

For illustration, the noise $\mathrm{n}$ is taken to be zero-mean and uncorrelated between sensors, with a covariance given by $\mathbf{K}_{n}=\beta^{2} \mathbf{I}$. Then the error covariance $\mathbf{K}_{e}$ is found by

$$
\begin{aligned}
\hat{\mathbf{p}} & =\mathbf{E}\left(\mathbf{E}^{+} \mathbf{E}\right)^{-1} \mathbf{E}^{+}(\mathbf{E} \boldsymbol{\alpha}+\mathbf{n})=\mathbf{E} \boldsymbol{\alpha}+\mathbf{E}\left(\mathbf{E}^{+} \mathbf{E}\right)^{-1} \mathbf{E}^{+} \mathbf{n} \\
\boldsymbol{e} & =\hat{\mathrm{p}}-\mathbf{p}=\mathbf{E}\left(\mathbf{E}^{+} \mathbf{E}\right)^{-1} \mathbf{E}^{+} \mathbf{n} \\
\mathbf{K}_{e} & =E\left[\boldsymbol{e} \boldsymbol{e}^{+}\right]=\mathbf{E}\left(\mathbf{E}^{+} \mathbf{E}\right)^{-1} \mathbf{E}^{+} \mathbf{K}_{n} \mathbf{E}\left(\mathbf{E}^{+} \mathbf{E}\right)^{-1} \mathbf{E}^{+}=\beta^{2} \mathbf{E}\left(\mathbf{E}^{+} \mathbf{E}\right)^{-1} \mathbf{E}^{+}
\end{aligned}
$$

but,

$$
\beta^{2} \mathbf{E}\left(\mathbf{E}^{+} \mathbf{E}\right)^{-1} \mathbf{E}^{+}=\sum_{i=1}^{M} \frac{\beta^{2}}{\lambda_{i}} \mathbf{u}_{i} \mathbf{u}_{i}^{+},
$$

where $\mathbf{u}_{i}$ is the eigenvector associated with eigenvalue $\lambda_{i}$. The error obviously increases as $\beta$ increases. More importantly, as $\mathbf{E}$ becomes more singular, the lowest 

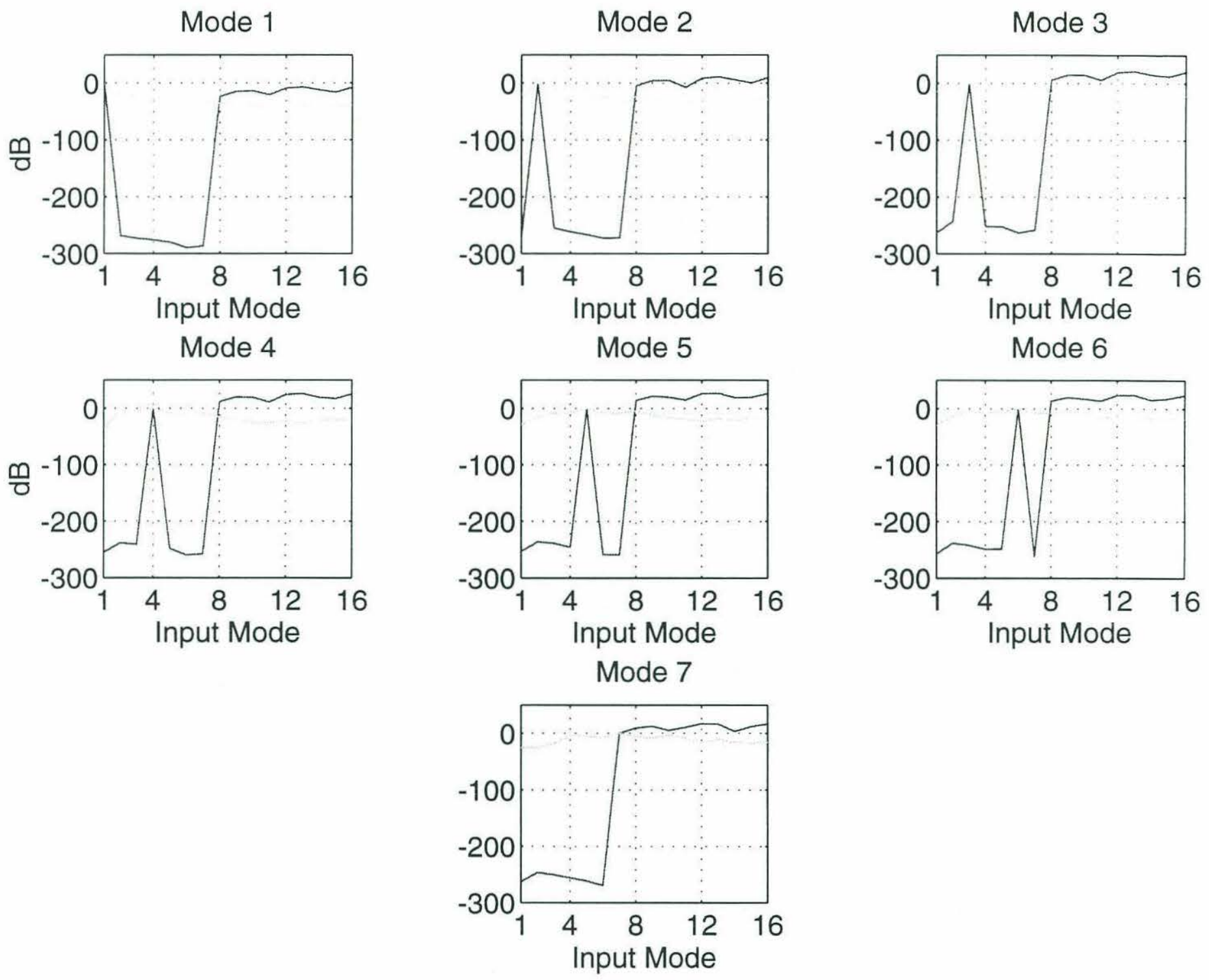

Figure 3-2: Least squares and single-mode beamformer responses for steering modes 1-7, and data channels 1-14. (dashed line: single-mode, solid line: least squares) 


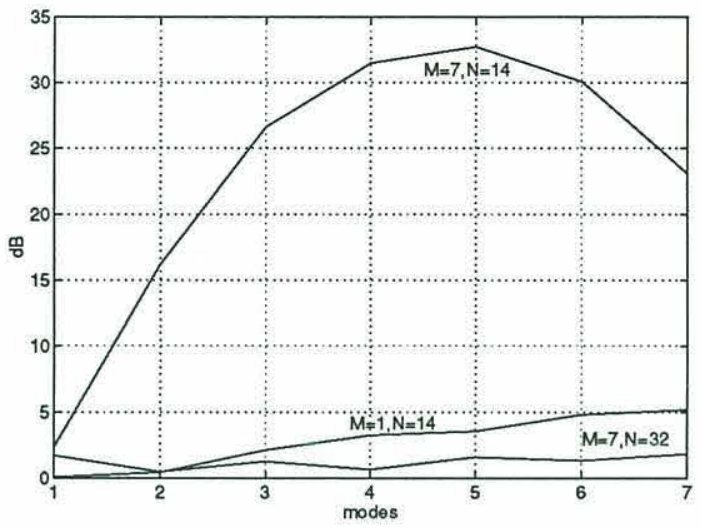

Figure 3-3: Response power for steering modes 1-7. $N$ is number of channels used, and $M$ is number of modes used in processor. $M=1$ indicates single-beam processor.

eigenvalue will tend towards zero, and the error covariance (as well as the error itself) goes to infinity.

Before moving on, one more observation may be made concerning the noise and the beampattern response. The spatial noise field can be expanded on the set of normal modes in a Karhunen-Loeve expansion, where $\beta_{i}$ is the amount of mode $i$ present in the noise field, $\mathbf{n}$.

$$
\mathrm{n}=\sum_{i=1}^{\infty} \beta_{i} \phi_{i}
$$

Using this expansion, there are two ways in which noise can interfere with mode estimates. One is that the noise associated with the first $M$ modes is lumped in with the $\alpha$ 's. Here, the only hope is that the SNR is high enough so that $\alpha_{i}>\beta_{i}$ for $1 \leq i \leq M$. The other way is that since the least squares processor can only reject modes less than mode $M+1$, any expression of the noise in modes higher than mode $M$ can dominate the estimates through singularities in computing the inverse. The extent to which this is a problem depends on the response pattern of the particular beamformer, and as shown above, the least squares beamformer, when limited to a small subset of sensors on the array, has a definite problem.

When only spatially white noise is used as the input signal, it is equivalent to 
uniformly exciting all of the modes. ${ }^{2}$ A distribution of coefficient powers similar to that in Figure 3-3 is obtained, with modes 3, 4, and 5 much higher than the others. Unless there is a way to reduce this bias, the least squares beamformer will have much poorer performance than the single-mode beamformer. To summarize, while the leakage from adjacent modes has been eliminated by using a least squares algorithm, there is now increased leakage from the higher modes that were not included in the processing. The next section discusses how the least squares may be modified to reduce this bias, making it more like the single-mode response while maintaining better rejection of the adjacent modes.

\subsection{Diagonal Loading}

Diagonal loading is a technique frequently used to counter the destabilizing effects of near-zero eigenvalues in the singular value decomposition. Here, loading is used to reduce the response contribution due to the presence of energy in higher modes not included in the least squares basis set. In the geophysical literature, this method is referred to as damped least squares [23]. As the name suggests, diagonal loading is accomplished by adding an amount $\epsilon$ along the diagonal of the modal covariance matrix, $\mathbf{K}_{\phi}=\mathbf{E}^{+} \mathbf{E}$. This is the same as adding $\epsilon$ to each of the eigenvalues of the covariance matrix. One way to compute $\epsilon$ is

$$
\epsilon=\frac{\gamma}{M} \operatorname{tr}\left(\mathbf{K}_{\phi}\right)=\frac{\gamma}{M}\left(\lambda_{1}+\lambda_{2}+\cdots+\lambda_{M}\right)
$$

where $\gamma$ is variable and is usually expressed as a percentage [24]. The effect of damping is best seen by considering the bias and variance of the new coefficient estimates. If the received signal is assumed to be of the form in (3.7), then the bias

\footnotetext{
${ }^{2}$ To be rigorous, this is true only in the limit of a continuous aperture over the entire water column.
} 
is

$$
\begin{aligned}
\text { bias } & =E[\boldsymbol{e}]=E[\boldsymbol{\alpha}-\hat{\mathbf{a}}]=E\left[\boldsymbol{\alpha}-\left(\mathbf{E}^{+} \mathbf{E}\right)^{-1} \mathbf{E}^{+}(\mathbf{E} \boldsymbol{\alpha}+\mathbf{n})\right] \\
& =\boldsymbol{\alpha}-\left(\mathbf{E}^{+} \mathbf{E}\right)^{-1} \mathbf{E}^{+} \mathbf{E} \boldsymbol{\alpha} \\
& =\mathbf{V}\left(\mathbf{I}-\boldsymbol{\Sigma}_{d}^{-1} \boldsymbol{\Sigma}\right) \mathbf{V}^{+} \boldsymbol{\alpha}
\end{aligned}
$$

The matrix $\boldsymbol{\Sigma}$ is the diagonal eigenvalue matrix from the singular value decomposition, $\mathbf{E}^{+} \mathbf{E}=\mathbf{V} \boldsymbol{\Sigma} \mathbf{V}^{+}$, and $\boldsymbol{\Sigma}_{d}$ is the diagonally loaded version. The term in parentheses then becomes

$$
\left(\mathbf{I}-\boldsymbol{\Sigma}_{d}^{-1} \boldsymbol{\Sigma}\right)=\left[\begin{array}{ccc}
\frac{\epsilon}{\lambda_{1}+\epsilon} & & \\
& \ddots & \\
& & \frac{\epsilon}{\lambda_{M}+\epsilon}
\end{array}\right]
$$

When $\epsilon=0$, the estimates are unbiased, and as the loading increases, $\boldsymbol{\Sigma}_{d}$ tends to zero and the bias becomes simply the expected value of the received signal. A consequence of this bias is that the unity gain constraint on the main response axis is violated. There are numerous techniques that alleviate this problem [21, 24]. One option is to adjust only those eigenvalues that are closest to zero. This leaves the estimates associated with the larger eigenvalues unbiased. These options were considered, but the results indicate that for the given situation, such variations have minimal effect on estimates of the lower modes. Table 3.1 shows the computed eigenvalues, their relative weightings, and how they would change using $20 \%$ diagonal loading.

The error variance for the loaded least squares is given by:

$$
\begin{aligned}
\operatorname{Var}(\boldsymbol{e}) & =E\left[\boldsymbol{e} \boldsymbol{e}^{+}\right]-E[\boldsymbol{e}] E[\boldsymbol{e}]^{+} \\
& =\sigma_{n}^{2}\left(\mathbf{E}^{+} \mathbf{E}\right)^{-1}\left(\mathbf{E}^{+} \mathbf{E}\right)\left(\mathbf{E}^{+} \mathbf{E}\right)^{-1}=\sigma_{n}^{2} \mathbf{V} \boldsymbol{\Sigma}_{d}^{-2} \boldsymbol{\Sigma} \mathbf{V}^{+}
\end{aligned}
$$




\begin{tabular}{||c|c|c|c||}
\hline \hline Number & Eigenvalue & Percentage & Adjusted Percentage \\
\hline 1 & 9.7950898 & 0.2469163 & 0.1862151 \\
2 & 9.7880296 & 0.2467383 & 0.1861410 \\
3 & 9.6868158 & 0.2441869 & 0.1850779 \\
4 & 6.8616895 & 0.1729706 & 0.1554044 \\
5 & 3.3293859 & 0.0839277 & 0.1183032 \\
6 & 0.2078324 & 0.0052391 & 0.0855163 \\
7 & 0.0008344 & 0.0000210 & 0.0833421 \\
\hline \hline
\end{tabular}

Table 3.1: Modal covariance eigenvalues, their relative percentages, and the relative percentages after loading. $(\gamma=0.20)$.

$$
=\sigma_{n}^{2} \mathbf{V}\left[\begin{array}{ccc}
\frac{\lambda_{1}}{\left(\lambda_{1}+\epsilon\right)^{2}} & & \\
& \ddots & \\
& & \frac{\lambda_{M}}{\left(\lambda_{M}+\epsilon\right)^{2}}
\end{array}\right] \mathbf{V}^{+} .
$$

The diagonal loading, or damping, reduces the variance associated with each of the coefficient estimates. It is readily apparent from (3.13) that with $\epsilon=0$, the variance goes to infinity for estimates with near-zero eigenvalues.

While there is no quantitative method for determining what $\epsilon$ should be, one rule-of-thumb is to keep $\epsilon$ less than the smallest eigenvalue, $\lambda_{M}$. For this analysis, the value of $\epsilon$ was chosen to be the one that minimized the differences in output power at different steering modes, given the same input signal. Looking at Figure 34, a plot of the total response power as done earlier, the reduction in bias across the steering modes is clearly visible. For loadings above about $20 \%$, the only effect is to reduce to overall level of the mode coefficients. Consequently, the value chosen to use in the data processing is $20 \%$, or $\gamma=0.20$. 


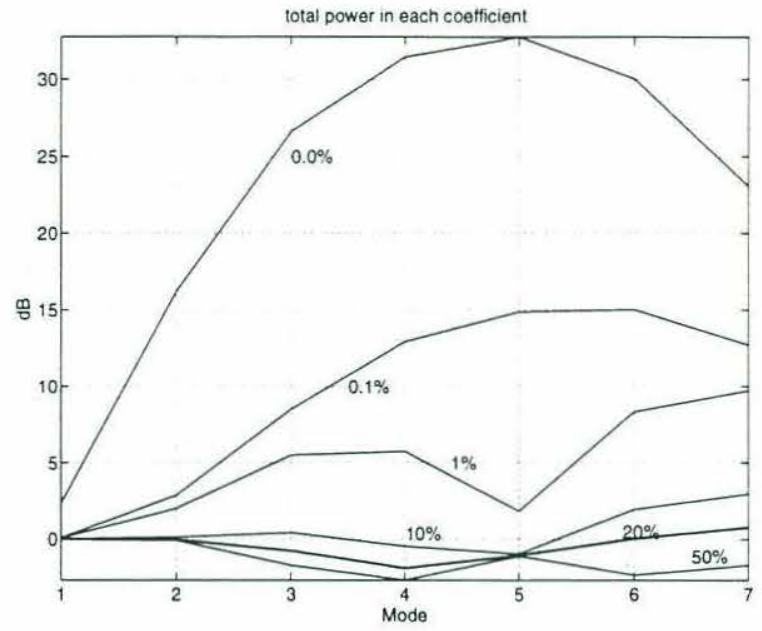

Figure 3-4: Total power for each mode coefficient for various levels of diagonal loading of the least squares processor. $(N=14, M=7)$

\subsection{Determining the number of modes}

The remaining issue now is determining $M$, the number of modes to include in the steering matrix $\mathbf{E}$. This has long been a problem in system identification and there has yet to be a satisfactory, optimal method for determining which modes to include [24]. The minimum number of modes to include is generally set by the number of modes anticipated in the signal, although due to array constraints even this minimum may not be attainable. The obvious maximum number of modes is dictated by the number of degrees of freedom, or in this case, the number of sensors, $N$. With $M<N$, the least squares problem is kept over-determined. In spite of this constraint, the steering matrix $\mathbf{E}$ can still become singular with the addition of more modes that are less and less orthogonal. An effective way of demonstrating this is to compute the effective singularity, or condition number, of the modal covariance matrix. Here $q$ is the effective singularity, and $M$ is the number of modes being used.

$$
q=\frac{\operatorname{det}\left[\mathbf{E}^{+} \mathbf{E}\right]}{\prod_{i=1}^{M}\left\|\mathrm{E}_{i}\right\|^{2}}
$$


Various curves of $10 \log (q)$ are plotted in Figure 3-5 for a range of diagonal loading values. Notice how the matrix conditioning rapidly decreases as more modes beyond mode 3 are included in the processor. This sharp decline is a result of the array sufficiently sampling only the first three modeshapes. (See Figure 4-1 for the actual

modeshapes) Without any diagonal loading, we can only be confident in resolving the first 3, or perhaps 4 modes. For $20 \%$ loading and the first 7 modes, $q$ is around -15 $\mathrm{dB}$, or 0.03 . Some have suggested a threshold of around $q=0.4$ [20], however, since each situation is different, the best way to determine how many modes to include is a combination of analytic results and trial and error with synthetic data.

\subsection{Performance}

Two items are of interest when looking at the performance of the least squares beamformer: the signal-to-noise ratio at which the estimates become indistinguishable, and whether it is possible to infer the actual array orientation from the acoustic data, since the true orientation is not known. Figures 3-6, 3-7 and 3-8 illustrate the least squares beamformer response in three different signal-to-noise environments. The procedure used in generating these figures is as follows. First, a synthetic signal was created using as an input the combination of modes 1,3 , and 5 in varying levels of background white noise. The phase of each mode is time-varying and randomized so that the resulting signal is a sum of incoherent modes. Four-hundred seconds of data were created at a sampling rate of $4.56 \mathrm{~Hz}$. The mode amplitudes were modulated by sinusoids with 200 second periods and phase shifts of 90 degrees with respect to one another. This makes it easier to see the crosstalk between modes. The signals were given a 100 second delay to simulate the arrival of a transmission. The indicated SNR values are those for a single channel, simply the ratio of the signal power to noise power. The estimated mode coefficients are plotted in a contour format, with 


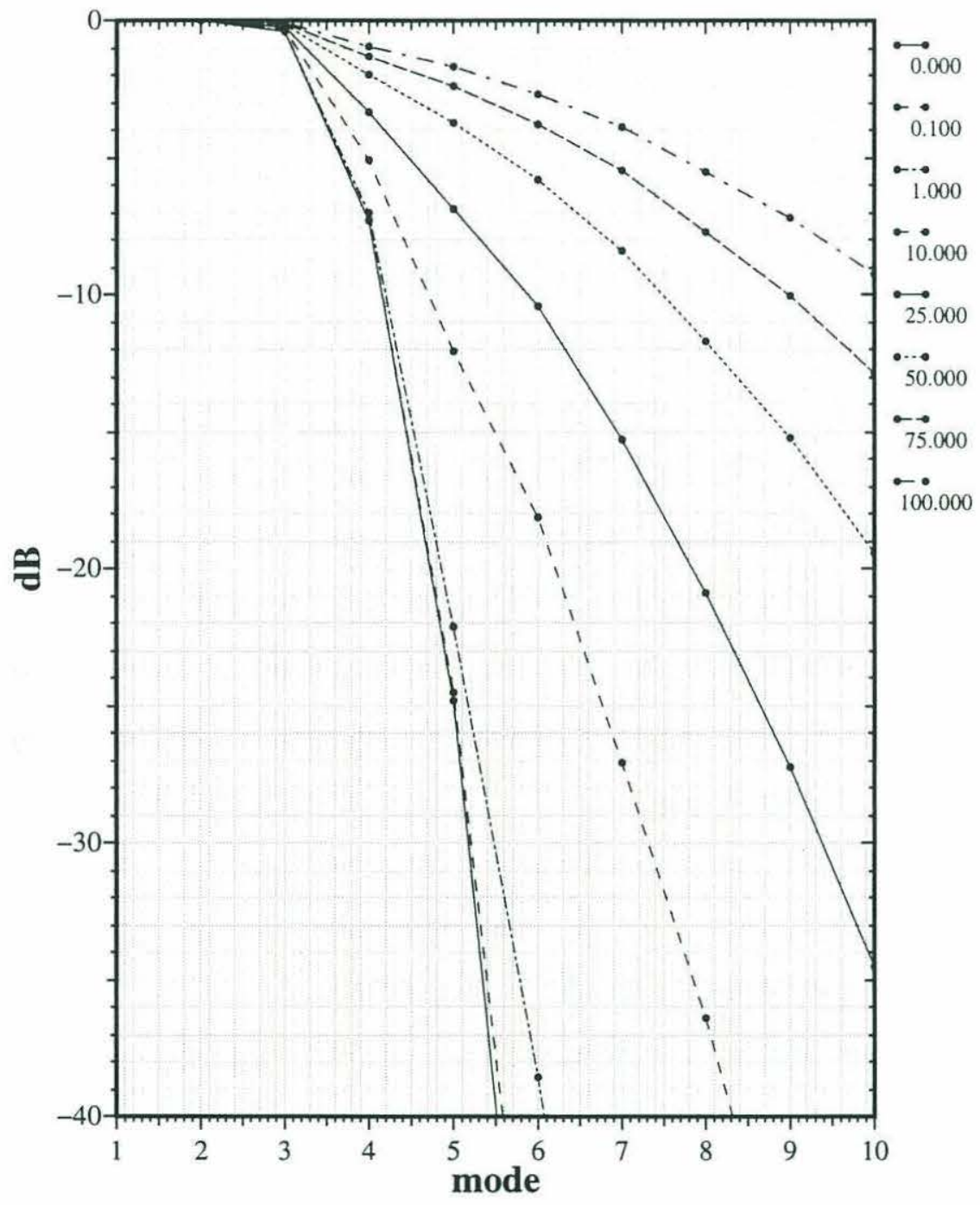

Figure 3-5: Singularity coefficients for the modal covariance matrix $\mathbf{K}_{\phi}$ at various levels of diagonal loading. 
mode number running along the $\mathrm{x}$-axis and time along the $\mathrm{y}$-axis. It is important to note that only integer mode numbers are physically allowed, and that anything in between is just an interpolation designed to aid in viewing the distribution of energy over the modes. Figure 3-6 shows the estimated mode coefficients given the input signal described above, with $20 \mathrm{~dB}$ SNR. Each mode was given the same level of excitation. The signal, arriving after 100 seconds, is clearly distinguishable. Also noticeable is the smearing of the energy, mode 5 into mode 6 in particular. This is a result of working with only 14 channels. Only the first three modes are completely sampled by the first 14 sensors, and so crosstalk between modes 4 and higher is unavoidable, no matter what processing technique is used. The signal is still apparent in the $10 \mathrm{~dB}$ SNR case, Figure 3-7, but the smearing is more pronounced. Finally, Figure $3-8$ shows that for $0 \mathrm{~dB} \mathrm{SNR}$, the situation is virtually hopeless. Without $a$ priori knowledge of the signal content, it would be difficult to get any picture of the signal. Only 6 modes have been used in the least squares beamformer. By eliminating mode 7 from the steering matrix, the conditioning was increased, which led to slightly better resolution of the modes. As the SNR is reduced, the higher mode estimates suffer the most degradation. In the $0 \mathrm{~dB}$ case, mode 5 is indistinguishable from the background noise. Fortunately from estimates in Chapter 2, the actual single-sensor SNR is around $10 \mathrm{~dB}$, post-processing, so the appropriate picture for the beamformer performance should be Figure 3-7.

In addition to looking at the performance versus signal-to-noise ratio, the sensitivity to array tilt and azimuth with respect to the direction of signal arrival was considered. Of specific interest is whether or not the acoustic data can be used to infer array orientation. By sweeping the least squares beamformer over a range of tilt and azimuth angles, the average fit, as given by Equation (3.6), can be tracked. The best fit over the range of angles should correspond to the correct array orientation. The success of this technique depends on how drastically the array-sampled 
modeshapes change with changing tilt and azimuth. The longer the array, the more these orientation changes will be reflected in the fit data and the more accurate the estimate of actual array position will be.

Figure 3-9 illustrates the least squares estimation fit, $10 \log (\eta)$, for three different array orientations. The actual array tilt was kept at $3.0^{\circ}$ from the vertical, and the relative azimuths between the array and direction of signal propagation were $150^{\circ}$, $90^{\circ}$, and $60^{\circ}$, going from top to bottom in the figure. The dashed line in each plot follows the maximum fit at each tilt angle. Notice that in each case, the actual array orientation falls directly on that line. When the signal-to-noise ratio is much lower, the fit may not be so close. Considering these plots further, the contour lines can be thought of as lines of constant phase; that is, the same relative phase shifts between sensors on the array are possible for a range of tilt/azimuth combinations. Note that the variations with depth of the mode magnitudes do not appear to be a factor in determining the level of estimation fit. If they were, then the level of fit would vary as one moved along an iso-phase line, changing the depths at which the sensors sampled the modeshapes but not the relative phases. This suggests that when the array is close to broadside to the incoming signal, where the tilt must be inferred solely from magnitude changes of the modeshapes, there will be more ambiguity in the actual orientation of the array. This can be seen as the relative azimuth approaches 90 degrees. The same level of fit is achieved for any of the tilt angles in the plot. 


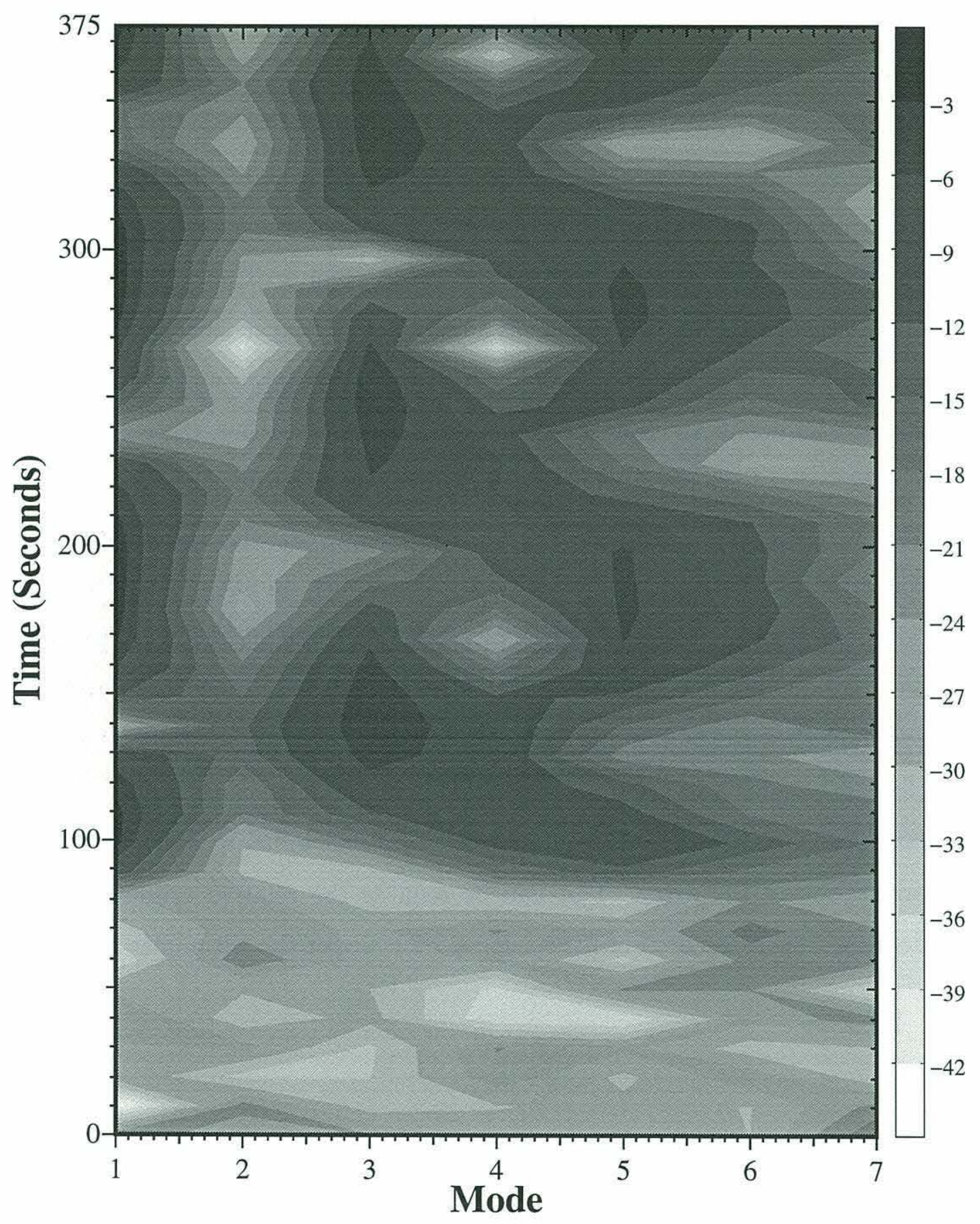

Figure 3-6: Normalized least squares beamformer response to synthetic data with 20 dB SNR. $(M=7, N=14, \gamma=0.20)$ 


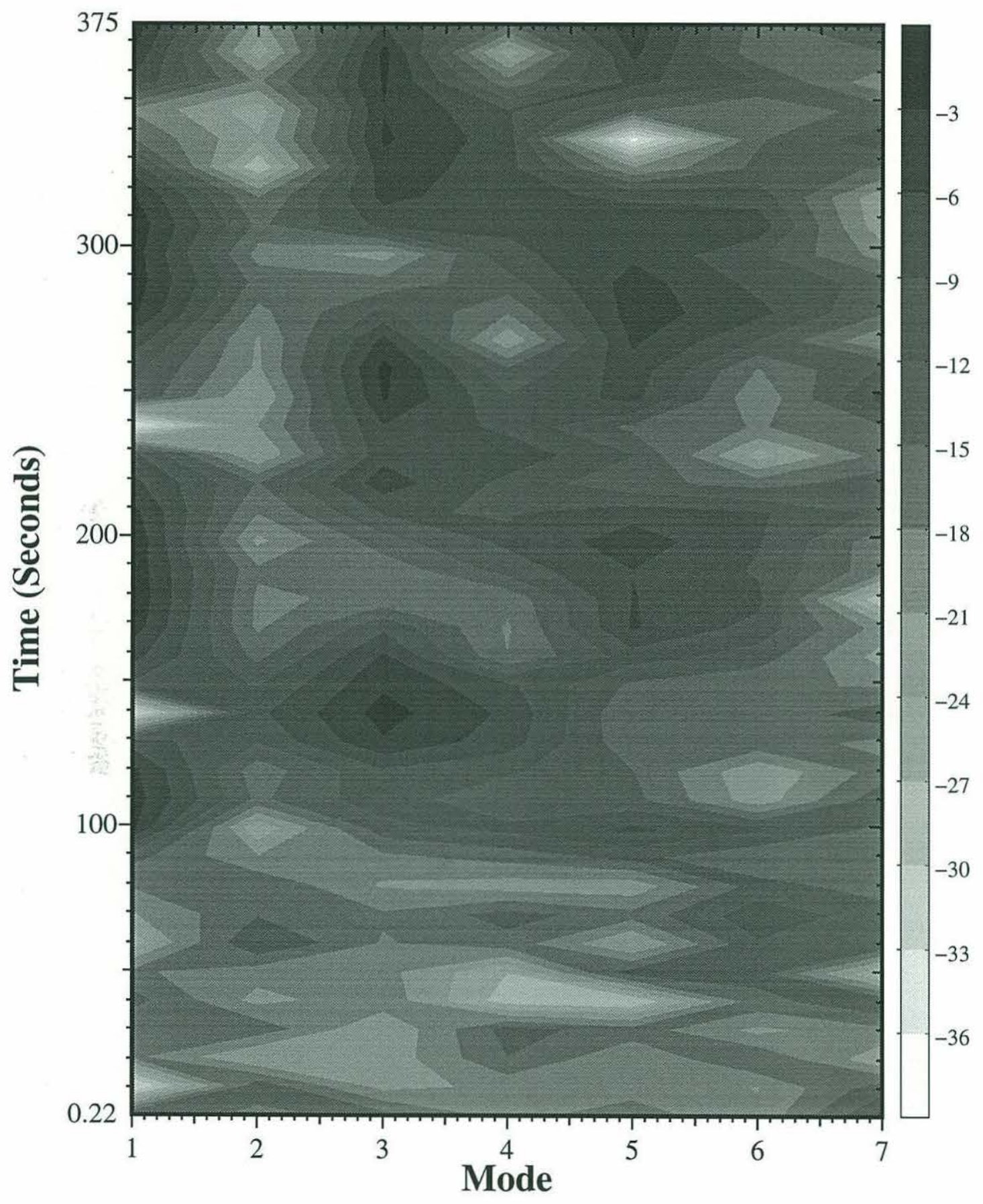

Figure 3-7: Normalized least squares beamformer response to synthetic data with 10 dB SNR. $(M=7, N=14, \gamma=0.20)$ 


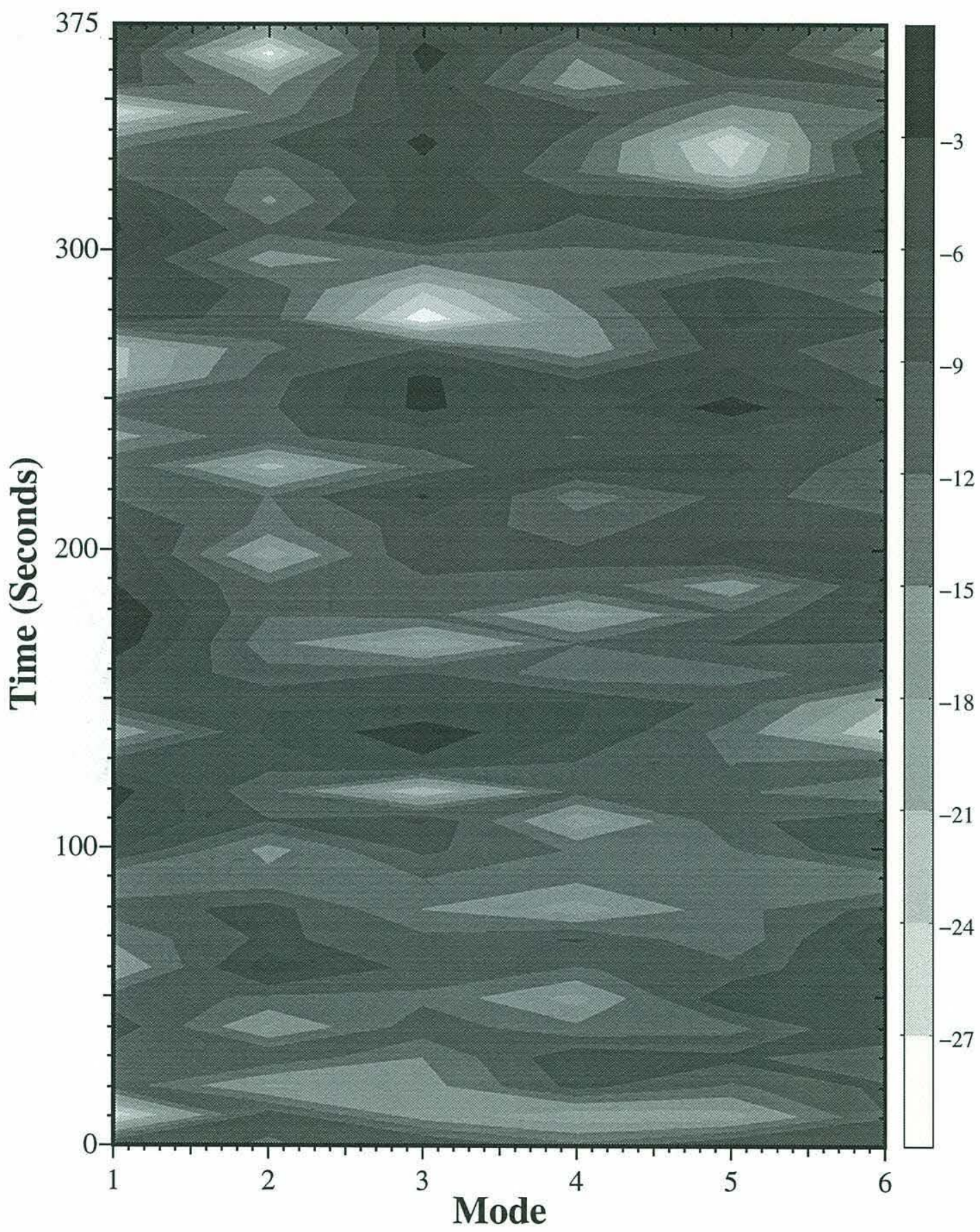

Figure 3-8: Normalized least squares beamformer response to synthetic data with 0 dB SNR. $(M=7, N=14, \gamma=0.20)$ 

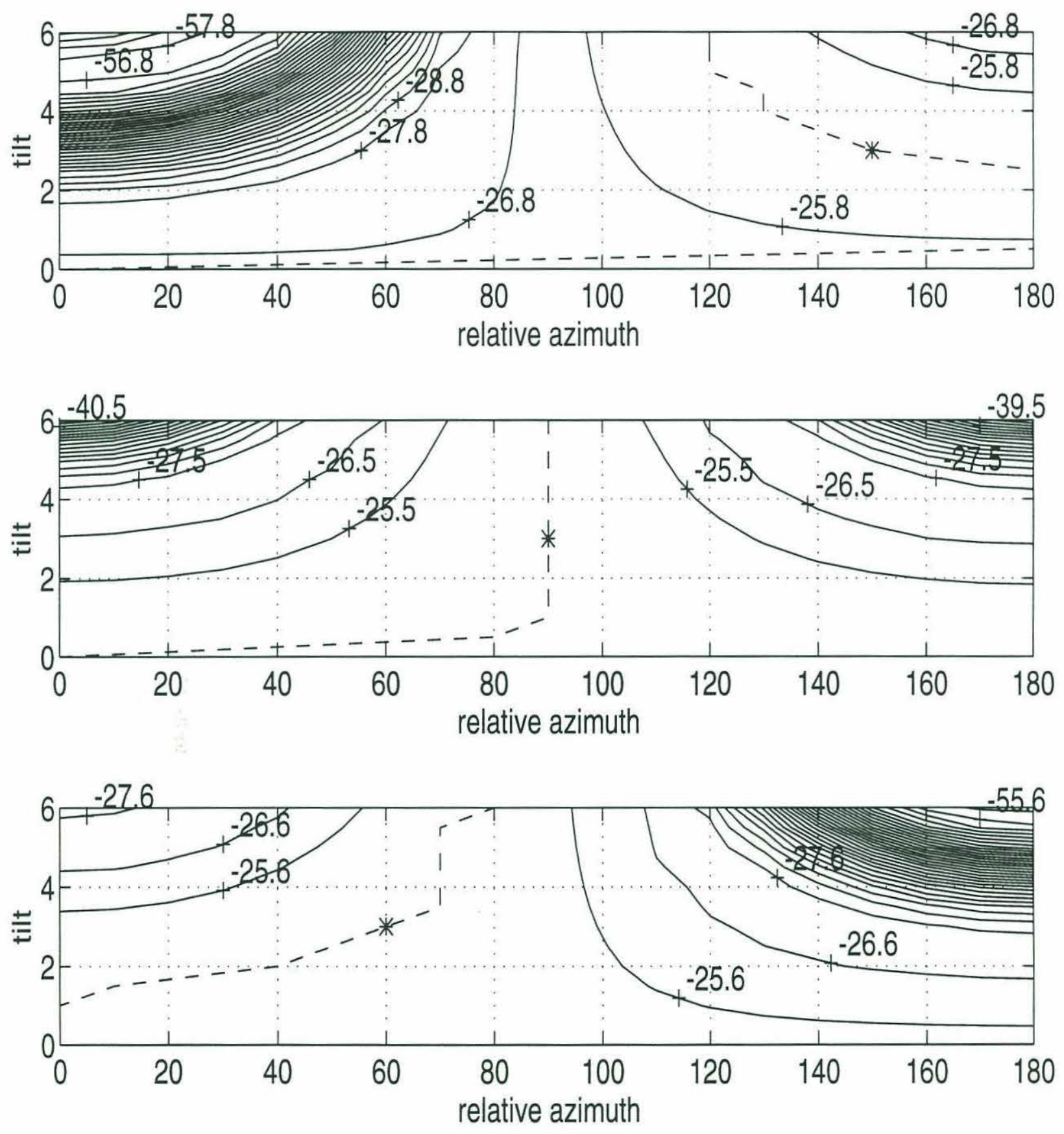

Figure 3-9: Estimation fits from synthetic data. Actual array orientations are indicated by ${ }^{*}$ 's. Least squares processing was done with $N=14, M=7, \gamma=0.20$. Modes 1,3 , and 5 were present with $10 \mathrm{~dB}$ SNR. 
This page left blank. 


\section{Chapter 4}

\section{Modal Analysis}

In this chapter two different analyses of the three data sets are presented. Sonograms were formed to show the distribution of energy with depth and time, and the least mean squares modal beamformer was used to estimate the energy in each of the modes present. To determine the array orientation for the modal analysis, the beamformer was swept over possible tilt and azimuth angles and, in conjunction with data from tilt sensors on the array, the most likely array orientation was determined for each dataset. The resulting conclusions are compared to those made from previous analyses performed on the same datasets, as well as the predicted results

from modeling done prior to the HIFT. First, however, this chapter begins with a discussion of the computed modeshapes at the vertical array, which form the basis for all of the subsequent analyses.

\subsection{Normal Mode Data}

Prior to deploying the vertical array, CTD casts were taken to sample the local sound speed profile. The profile, shown on the left in Figure 4-1, is typical of a winter profile for the area. The mixed layer extends for about 100 meters and the 
minimum is around 550 meters. The homogeneous wave equation may be solved numerically to give the modeshapes and modal group and phase velocities. There are numerous techniques available that accomplish this. The method used here was one developed by Baggeroer [25], which uses a modified integration technique based on the Prufer transformation. The first twelve computed modeshapes are shown on the right in Figure 4-1. Horizontal lines illustrate how the operational sensors sample the modes. This is important in interpreting the upcoming results. Notice that only the first three modes are sufficiently sampled by the 14-sensor subarray.

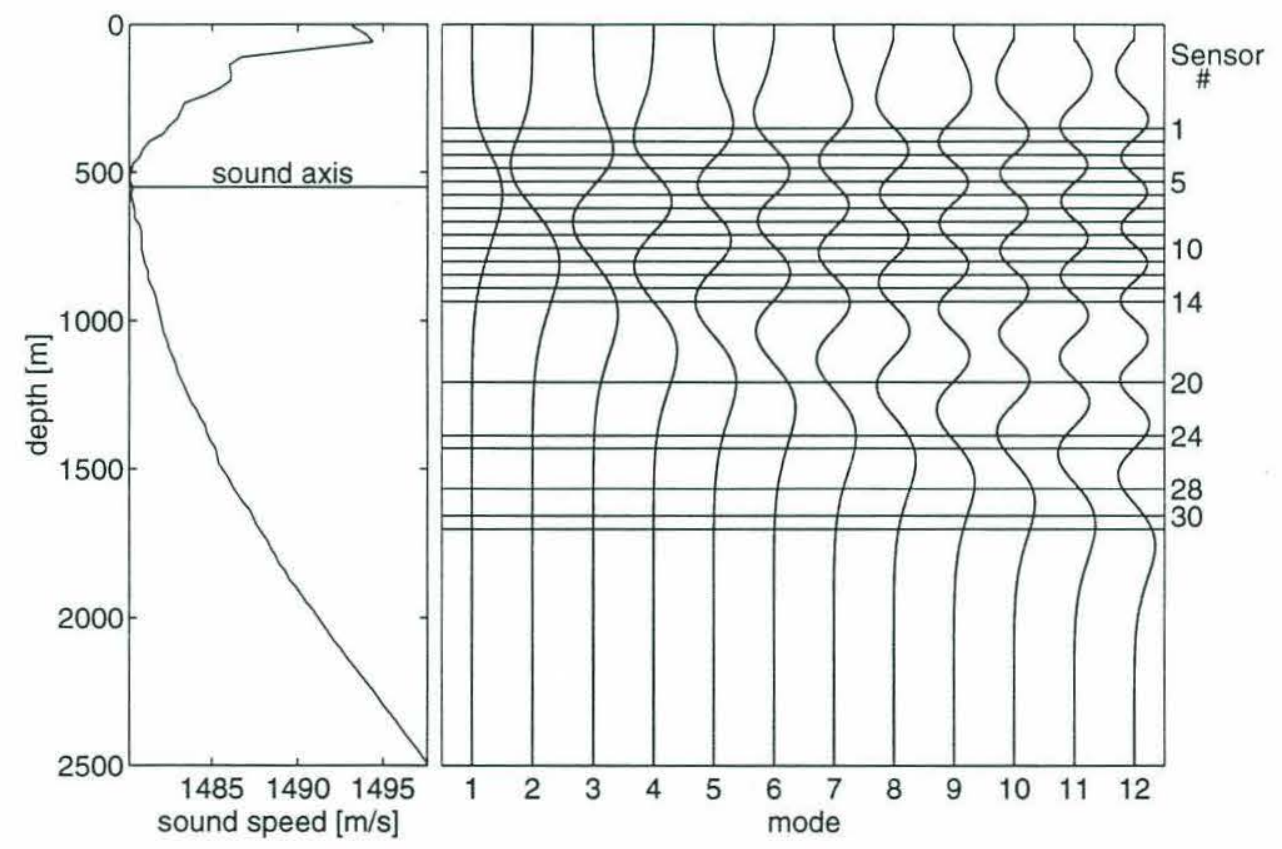

Figure 4-1: Sound speed profile and computed modeshapes at the Monterey site. Horizontal lines indicate active sensor depths.

\subsection{Sonograms}

A particularly straightforward method of presenting the data is to form a sonogram for each channel on the array, showing the signal energy versus frequency and time. 
To form the sonograms, short-time FFT's were formed from the data. A 200 second Hamming window was used with 100 second advances between FFTs. The resulting sonograms for each of the three CW transmission events are shown in Figures 4-24-5. Each column of boxes represents a different dataset, and each row is from the same sensor, with depth indicated on the left. The sensors for the 01270322 event are actually $12 \mathrm{~m}$ higher than the depths shown on the left. Each individual box has time along the $\mathrm{x}$-axis and deviation from the Doppler-corrected carrier frequency along the $y$-axis. The arrival of the signal is apparent across channels for each data set, although the delay from start of recording to signal arrival varies for each dataset. The pre-processing has added a 300 second delay to the beginning of each data set which has not been removed in these figures.

The plots illustrate several characteristic features of the data. First, there is about a $12 \mathrm{~dB}$ difference between the brightest red peak and the blue background. This is consistent with earlier single-channel SNR estimates. As before, the dominant feature is the fading in and out of the signal with time. Another apparent feature is the lack of tracking between adjacent channels that one would expect to see, given the slowly-varying nature of the lower modes with depth. Possible explanations for this include coherent interferences between modes that occur or various oceanographic phenomena. Another possibility is the fact that the propagation of a signal through the Antarctic Convergence Zone is quite complex and depends on how the signal is incident upon the front. Movement of the source ship relative to the stationary front might affect how the signal emerges from the front. While it is difficult to determine the exact cause of the fluctuations, it is known that similar oscillations were seen in data at other sites during HIFT [26].

The most interesting observation to be made from the sonograms is that there is significant energy present on even the lowest channels. The assumption prior to HIFT was that only the lowest few modes would be present at 18,000 km. Event 1505, 
though, has significant energy down to 1500-1600 meters. Referring to Figure 4-1, it is apparent that energy seen on sensors 20 and deeper can only come from modes 5 and higher. In the 1505 case, the energy at 1650 meters could possible come from mode 10 or higher. The implication, then, is that there are actually more modes present at the array than were originally anticipated. In the next sections, the least squares decomposition is used to estimate the relative strengths of the modes present. 


\section{Transmission Event}

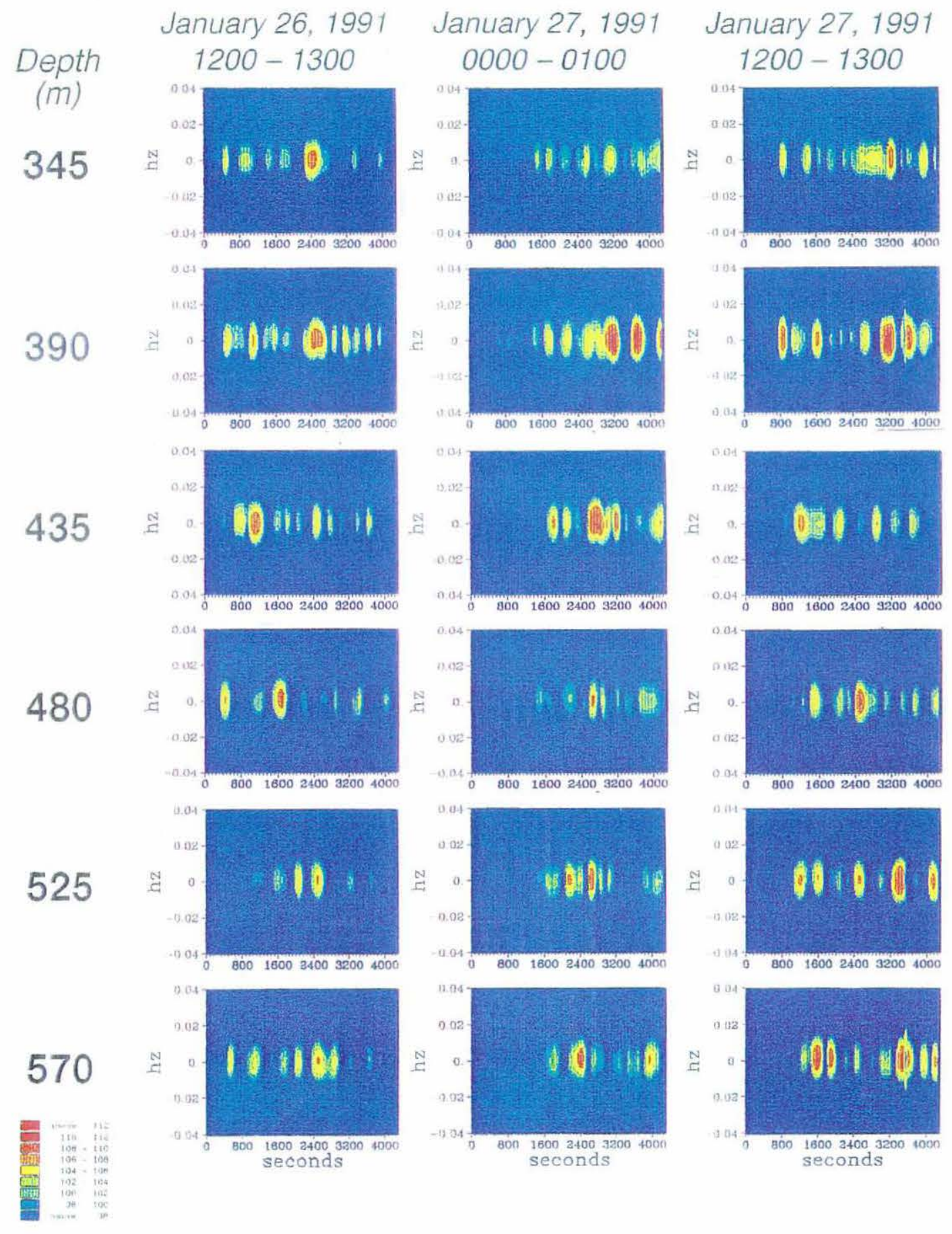

Figure 4-2: Sonograms from hydrophones 1-6 on the VLA. Each column is from a particular transmission, and each row is from the same hydrophone. 
This page left blank. 


\section{Transmission Event}

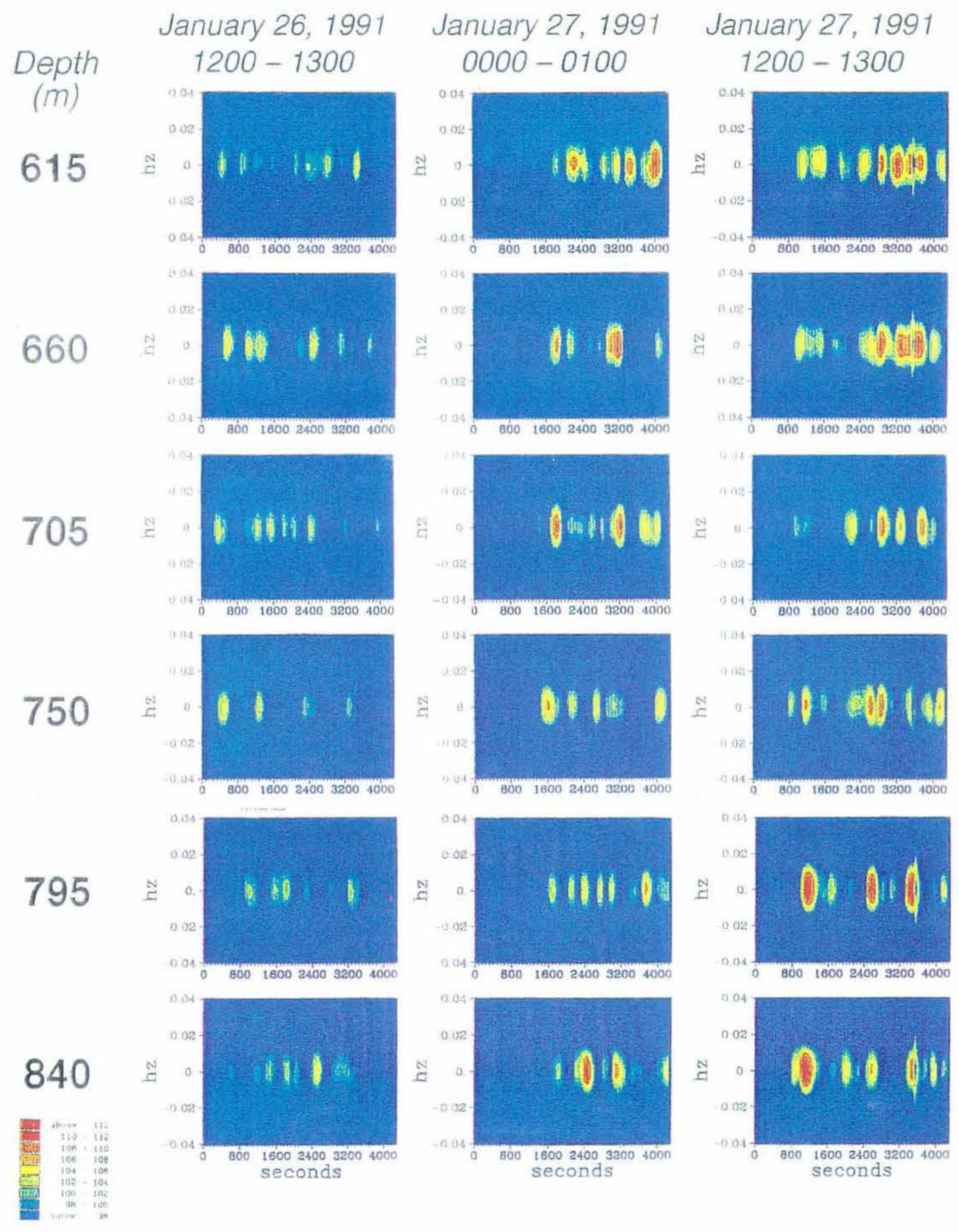

Figure 4-3: Sonograms from hydrophones 7-12 on the VLA. Each column is from a particular transmission, and each row is from the same hydrophone. 
This page left blank. 


\section{Transmission Event}

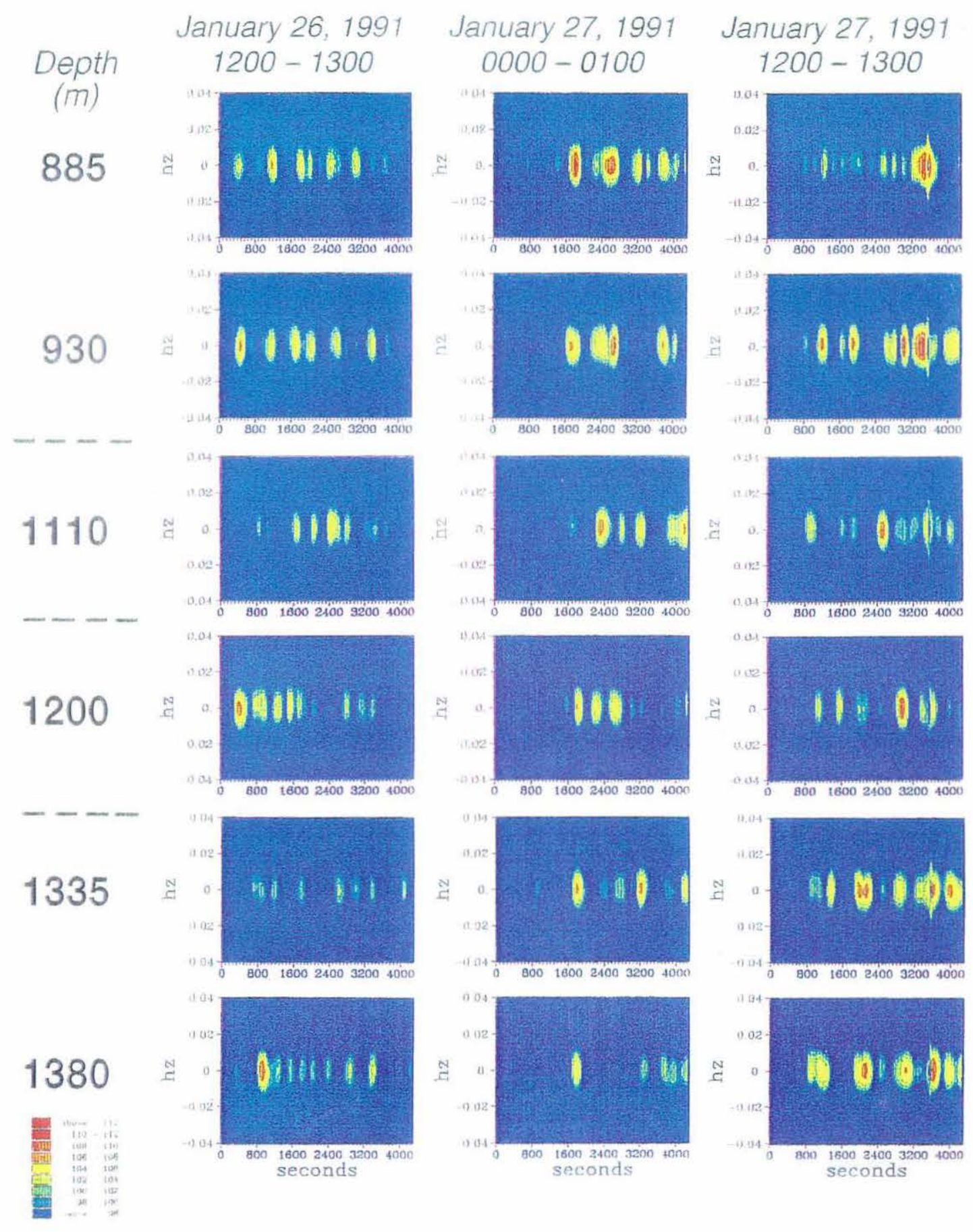

Figure 4-4: Sonograms from hydrophones 13, 14, 18, 20, 23, and 24 on the VLA. Each column is from a particular transmission, and each row is from the same hydrophone. 
This page left blank. 


\section{Transmission Event}

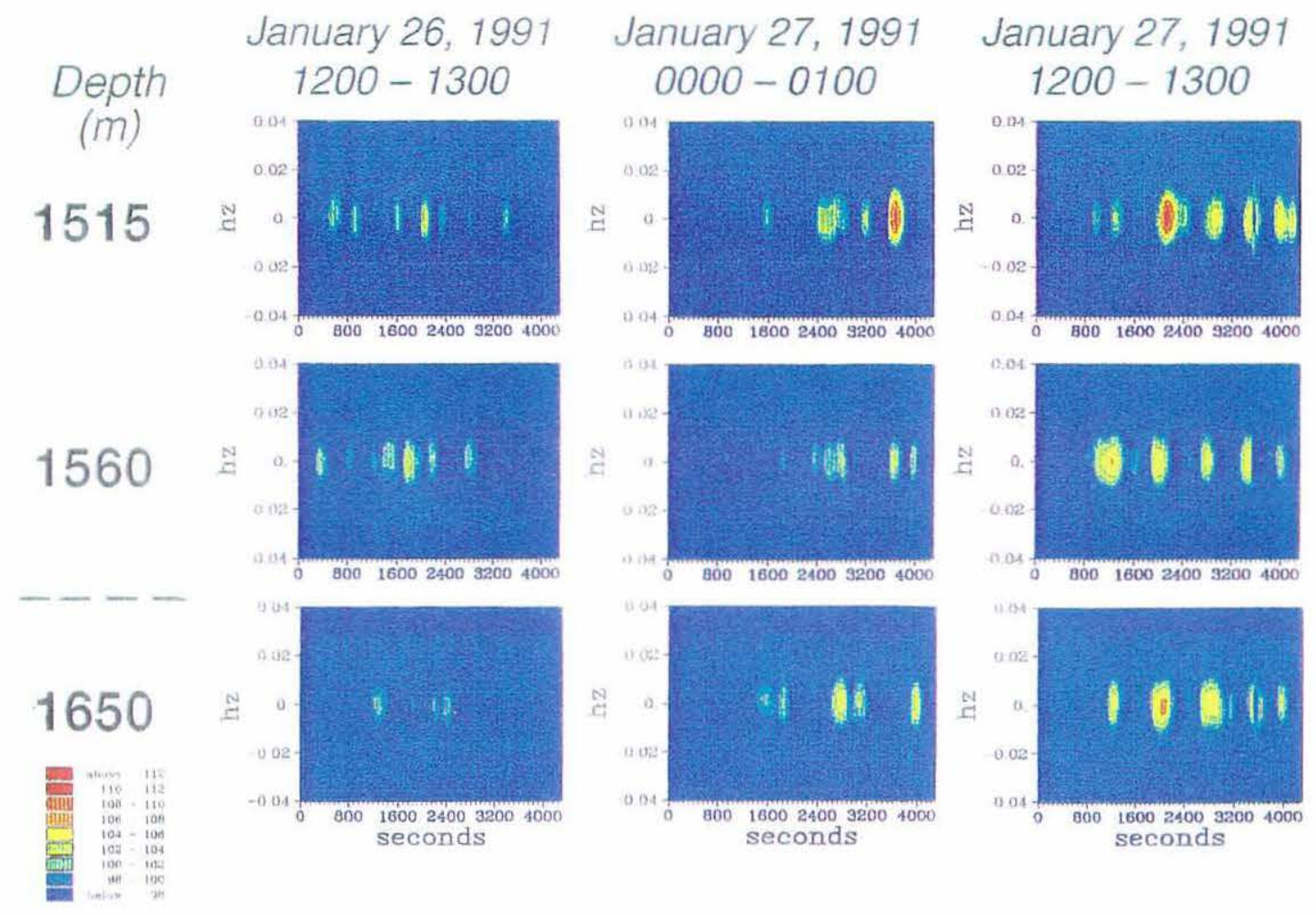

Figure 4-5: Sonograms from hydrophones 27, 28, and 30 on the VLA. Each column is from a particular transmission, and each row is from the same hydrophone. 
This page left blank. 


\subsection{Array Geometry}

Unlike the sonograms of the previous section, the least squares beamformer includes information about the phase of the signals. For each mode to be accurately estimated, the relative sensor displacements along the direction of propagation must be known. To this end, the Monterey array was equipped with tilt, depth and azimuth sensor packages, one 4 meters above the first hydrophone and another 5 meters below the number 20 hydrophone. Unfortunately, neither of the two azimuth sensors nor the deep tilt sensor functioned properly. Valid data was obtained, however, from the upper tilt and the two depth sensors. Figures 4-6 and 4-7 display the output of the upper sensors, with time referenced to the start of the acoustic data recording. The 01261525 and 01271505 data sets were quite stable throughout the time of data reception, unlike during the 01270322 reception, where the the array was repeatedly pulled upwards in the water column.

To simplify the analysis, the array is assumed to be straight with a slight tilt in the vertical. Because of currents and ship movement, there is a good chance that the array was not actually straight, so it is important to estimate the array's deviation from a straight line. Inferences as to the straightness are made by computing the expected depth at the lower sensor based on a straight array and the measured upper tilt angle. This can be compared to the lower depth sensor output, as Figure 4-9 shows. The measured lower depth is about 5 meters higher than expected, indicating the array was bowed slightly. The schematic in Figure 4-8 illustrates possible array orientations. Part (a) shows the horizontal projection of the array, $\Delta r$, in relation to the incoming signal. Part (b) indicates how array curvature affect the horizontal projection. The maximum possible horizontal displacement at sensor 20 that would give the the same depth as measured is 90 meters. Using the measured angle of $1.5^{\circ}$ and assuming a straight array, the displacement ( $\Delta r$ in Figure 4-8) is only 23 meters. 
At $57 \mathrm{~Hz}$, the wavelength is 26 meters, which means phase errors of over a complete cycle are possible. Fortunately, it appears that the array was more broadside to the incoming signal, greatly reducing the phase differences between sensors. Still, because of the potential for serious phase errors, the analysis is limited to the first fourteen sensors. This array subset also happens to be the only contiguous set of sensors out of the 21 that had acceptable data quality, as discussed in Chapter 2.

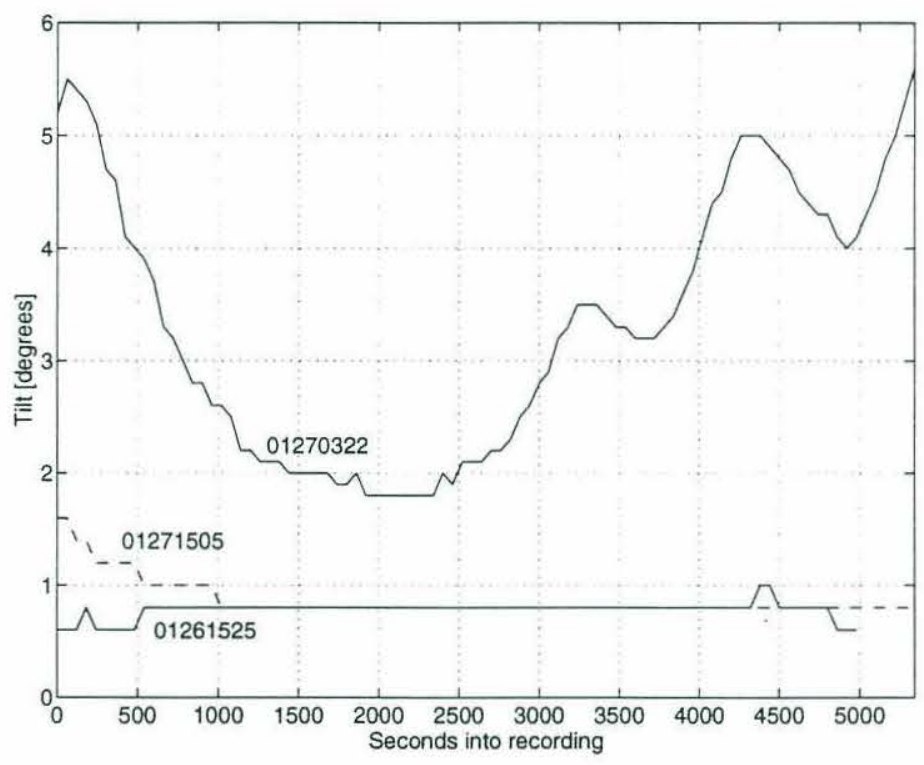

Figure 4-6: Output from upper tilt sensor. Time is referenced to start of each acoustic data set.

Since no azimuth readings were obtained, it is difficult to tell exactly where the array was pointing. Based on the ship's drift track and local wind and currents in the area, a relative angle of 60 degrees with the signal path from Heard Island is reasonable [26]. Figures 4-10, 4-11 and 4-12 show plots of the least squares estimation fit as discussed in in Chapter 3. The y-axis shows the tilt angle, defined to be the angle the array makes with a vertical line dropped through the top sensor. Along the $\mathrm{x}$-axis is the relative angle between the arriving signal and the array. The dashed line in the plots connects the points that gave the best fit for each tilt angle. If the 


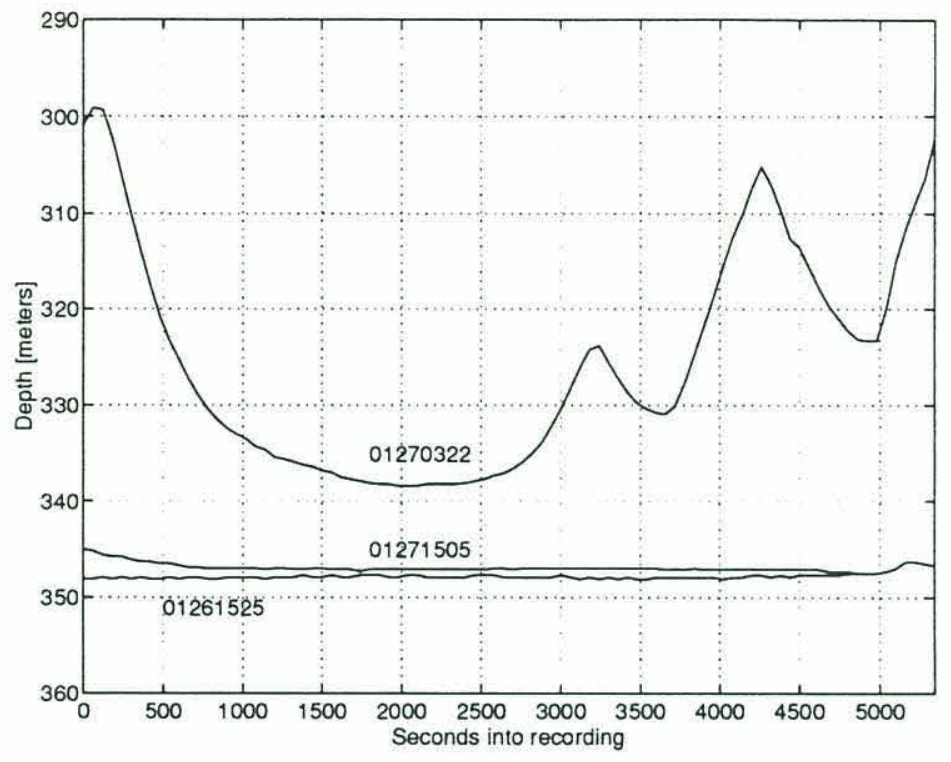

Figure 4-7: Output from upper depth sensor.

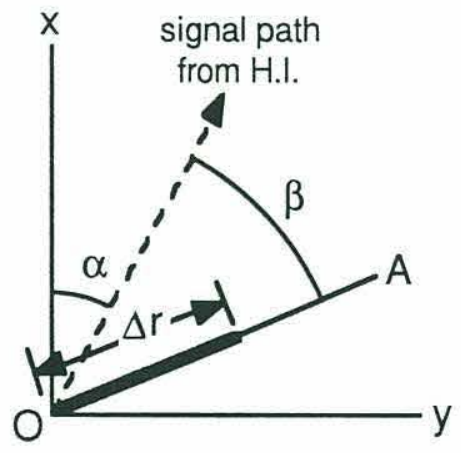

(a)

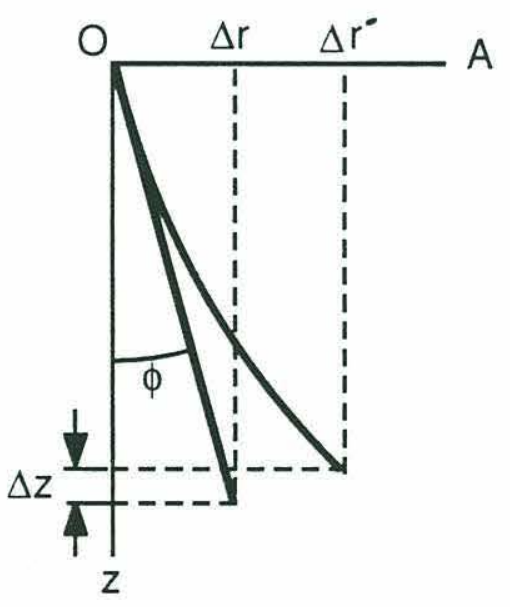

(b)

Figure 4-8: Schematic of array orientation. (a) $\alpha$ is the signal azimuth, $\beta$ the relative azimuth between array and signal, $\Delta r$ the array projection onto the $x-y$ plane. (b) shows two possibilities for vertical array tilt having the same upper tilt angle $\phi$. 


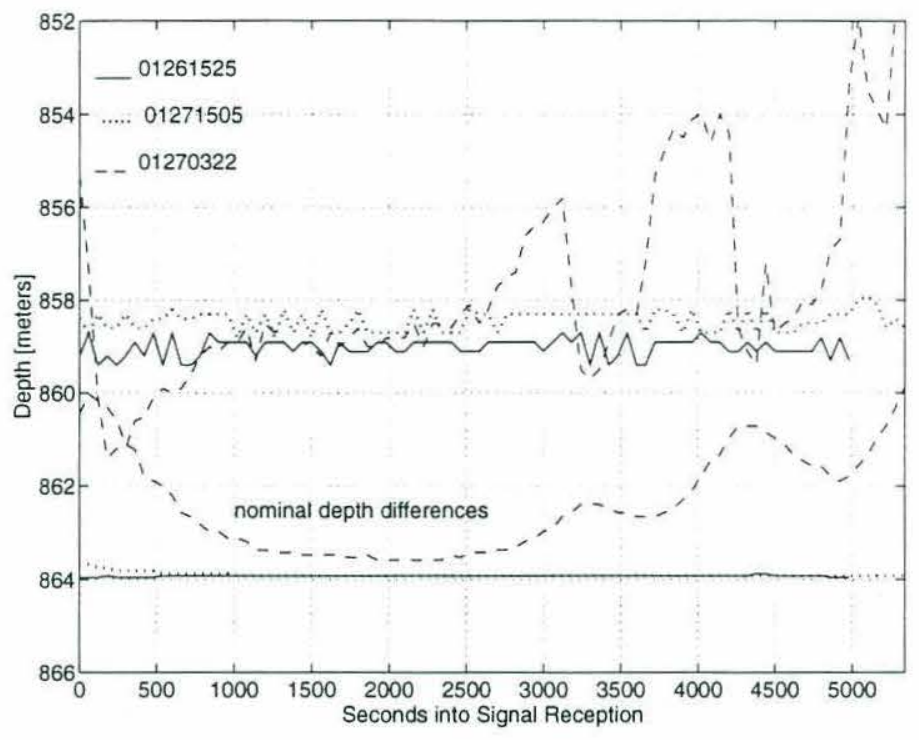

Figure 4-9: Difference between upper and lower depth sensors. Nominal differences are shown based on upper tilt measurements.

upper tilt sensor accurately reflected the tilt of the upper portion of the array, the intersection of this angle with the azimuth as inferred from the ship's drift yields a point that is reasonably close to the best-fit line in the figure, and certainly within the upper contour level. Since the 60 degree approximation is a rather general approximation, the azimuth corresponding to the best-fit point will be used. Along with this, a one-half degree tilt will be added to make a better linear approximation to the array curvature.

The azimuth angles are within $\pm 40^{\circ}$ of estimated azimuth. For the 1525 transmission, the bottom of the array appears to be pointing back towards Heard Island, rather than away from it as in the other two datasets. The following table summarizes the estimated tilt and azimuth angles for each dataset. In these estimations, more weight has been given to the tilt data from the upper tilt sensor than to the inferred azimuth. 


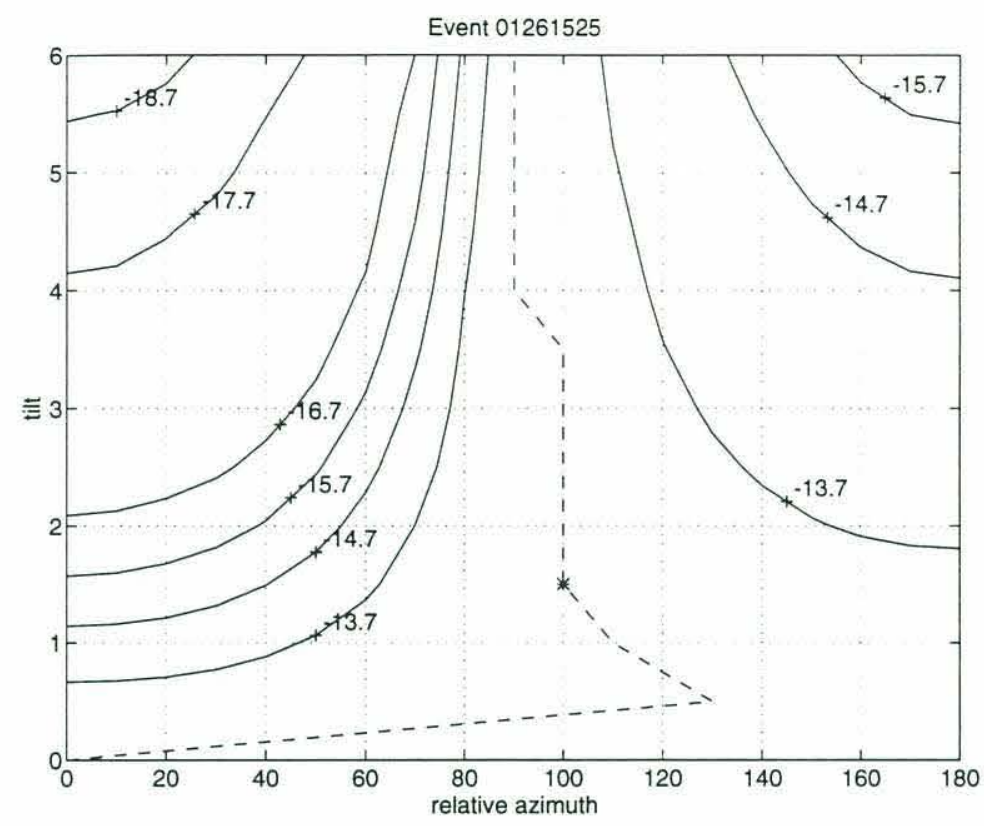

Figure 4-10: Event 01261525 estimation fit based on 7-mode least squares fit averaged over the period 0-2000 seconds. Asterisk indicates estimated array orientation.

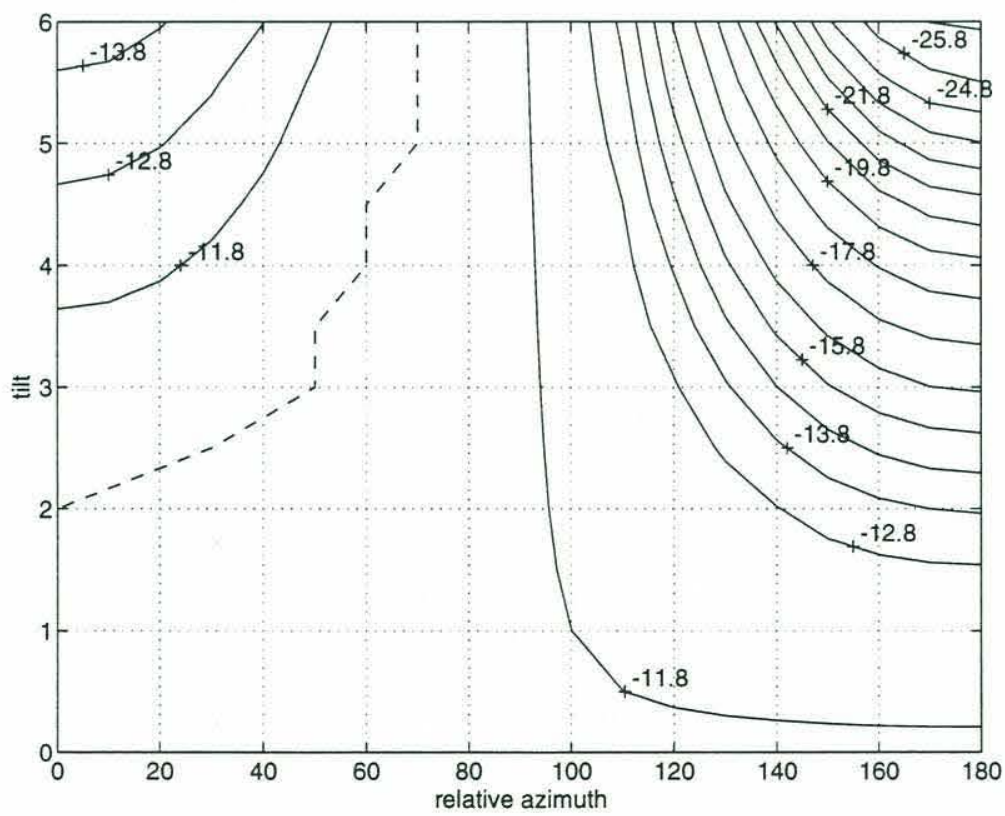

Figure 4-11: Event 01270322 estimation fit based on 7-mode least squares fit averaged over the period 1600-2400 seconds. Asterisk indicates estimated array orientation. 


\begin{tabular}{||c|c|c||}
\hline \hline Event & Estimated Tilt & Estimated Azimuth \\
\hline 01261525 & $1.5^{\circ}$ & $100.0^{\circ}$ \\
01270322 & $2.5^{\circ}$ & $30.0^{\circ}$ \\
01271505 & $1.5^{\circ}$ & $60.0^{\circ}$ \\
\hline \hline
\end{tabular}

Table 4.1: Estimated tilt and azimuth angles based on estimation-fit.

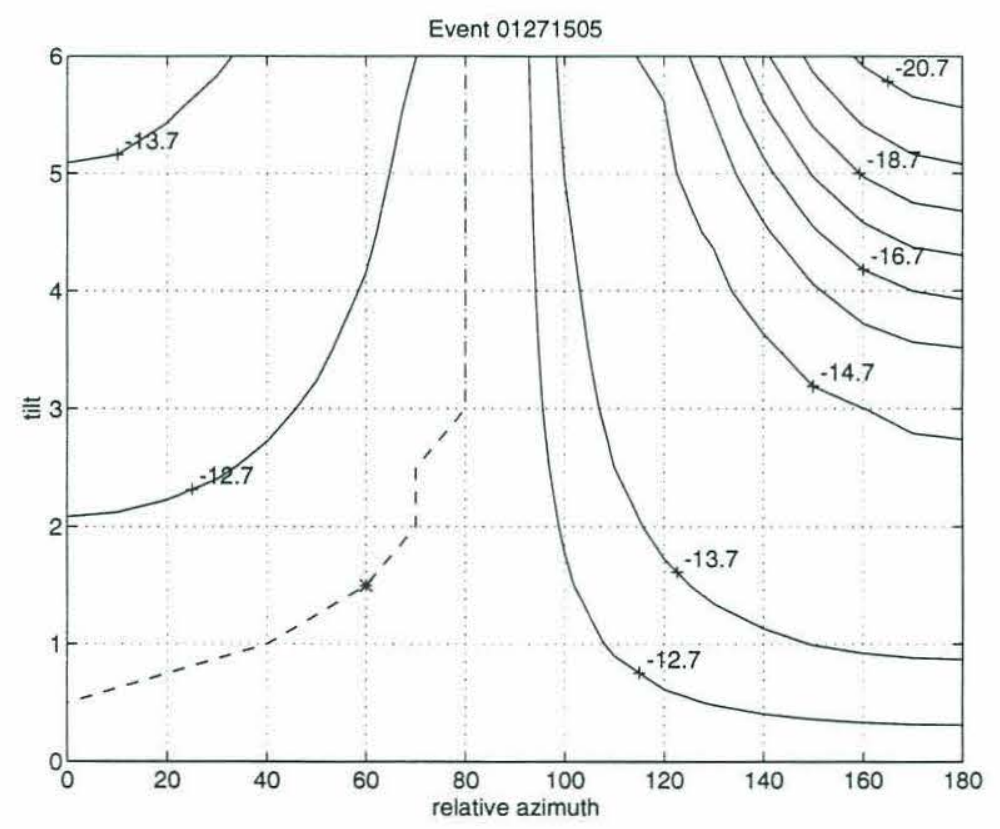

Figure 4-12: Event 01271505 estimation fit based on 7-mode least squares fit averaged over the period 1000-2500 seconds. Asterisk indicates estimated array orientation. 


\subsection{Modal Fitting}

Using the previously-determined orientations, estimates were made of the modal coefficients as a function of time. Modes 1-7 formed the basis set for the least squares decomposition, and only the top fourteen channels were used. Diagonal loading of

$20 \%$ was applied to prevent the estimates from being dominated by singularities arising from using a subset of the hydrophones. The fluctuating nature of the signal complicated the processing. It was found that 200 second window lengths worked best, with half-window length advances. This size of window is on the order of the final lowpass filter length. The results for the three datasets are shown in Figures 413, 4-14, and 4-15. Each mode coefficient is plotted separately versus time, with the resulting least squares estimation fit shown at the bottom of each page. The vertical lines indicate the predicted duration of the signal, and the coefficient mean and standard deviation over this period are indicated at the right of each figure.

Looking first at Event 01261525, it is encouraging to see the level of fit decrease substantially after the transmission end, although with the applied loading, the validity of the estimation error/fit is questionable. The best way of estimating the strength of each mode is to compute the rms value over the time period the signal is present. For this event, modes 3, 5, and 7 appear to have the highest energy. The energy in mode 7 supports what was seen in the sonograms, the possible presence of higher modes.

The 01270322 event is less conclusive than the 01261525 event. Modes 1, 2, and 5 show the highest levels. It is uncertain how much weight should be placed on measurements from this particular event, given that the array was being pulled around for most of the transmission. For event 01271505, modes 6 and 7 have the highest amplitudes, in agreement with what was seen in the sonograms. 


\subsection{Interpretation}

As was mentioned earlier, other analyses were done prior to this work on the same datasets. It is interesting to compare the results. A frequency vertical-wavenumber analysis was performed by Mikhalevsky [7] of SAIC utilizing the same conditioned datasets. The frequency-wavenumber analysis assumes plane-wave propagation and looks at the distribution of energy versus angle-of-incidence on the array, or equivalently, the vertical wavenumbers associated with such plane waves. While this analysis requires no information regarding array orientation, how the data is interpreted does depend on the assumed orientation. The essential parameter is the effective array tilt in the signal propagation plane, which is simply $\theta_{\text {eff }}=\tan ^{-1}(\tan \theta \cos \phi)$, where $\theta$ and $\phi$ are the actual array tilt and azimuth. For the 01261525 event, an effective tilt of $1.5^{\circ}$ places the strongest arrivals at angles corresponding to modes 3 or 4 , corresponding nicely to the least squares results. Energy was also seen at an angle that would correspond to mode 10 or so, which would account for the energy seen in mode 7 of the least squares. The frequency-wavenumber results for 1270322 suggest the presence of modes $3-4$, which does not agree well with the above results, however, a different orientation was assumed. If the wavenumber analysis is re-interpreted using the estimated orientation from earlier, then it is possible to get arrivals that correspond to modes 1 and 6. Similarly, re-interpreting for the 01271505 event gives the presence of modes 2 and 6 . These interpretations should not be regarded as being precise. Because of the nature of the data, one can only hope to show what is possibly happening, and to rule out the obvious, such as the existence of energy in only the first two modes.

Single-mode beamforming was done for the 01261525 event, looking at only the first five modes [7]. Mode 3 was shown to be the most energetic, almost $3 \mathrm{~dB}$ above

the other modes, which agrees with the least squares results, with the exception of 
mode 4. Extensive modeling was done by McDonald, et al. [27], using a combination of coupled mode theory and parabolic equation methods. Their modeling suggests that modes 5-6 should be the most energetic. While this is consistent with some of the results seen here, it should be noted that they did not incorporate potentially important factors such as modal scattering from internal waves.

To put these results into perspective, recall that the standard assumption made going into HIFT was that only the lowest few modes would be make it to Monterey. This is clearly not the case. The least squares modal beamforming and sonogram analysis of this thesis, as well as frequency-wavenumber analyses single-mode beamforming by others, all indicate the presence of higher order modes. These modes appear equally as strong as the lower ones, if not higher. 


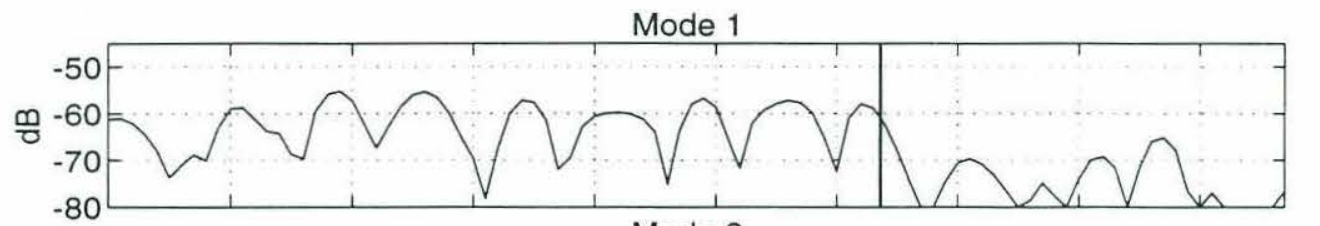

Mean S.D.

(dB)

$-603$

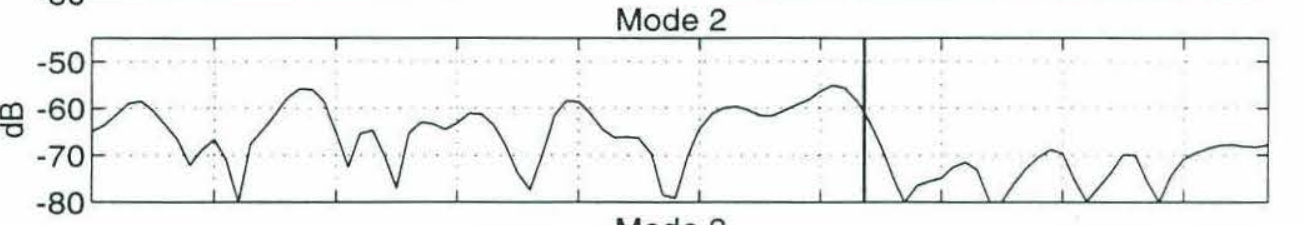

$-613$

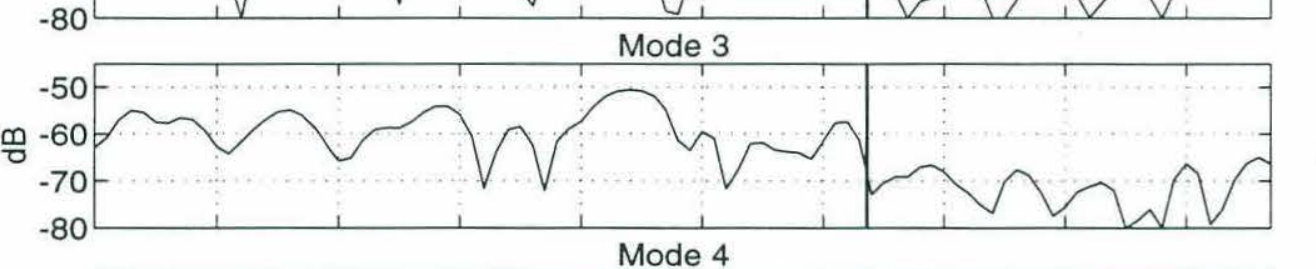

$-563$

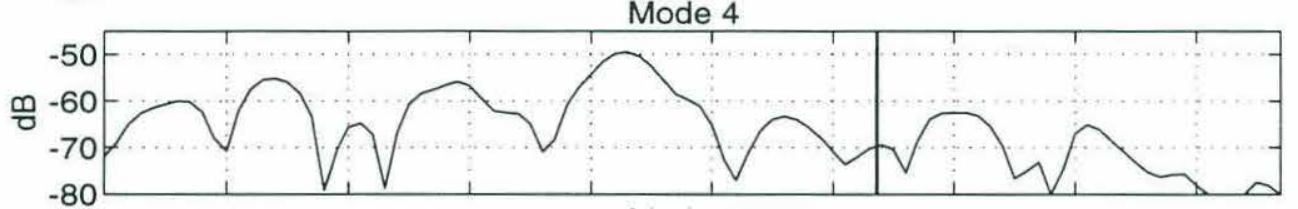

$-56 \quad 5$

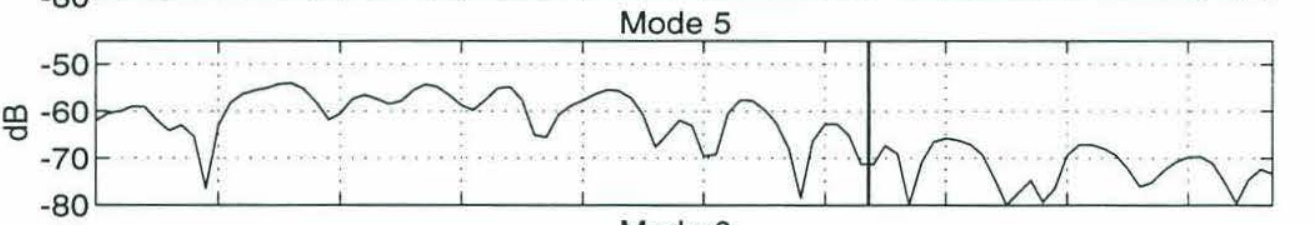

$-57 \quad 2$

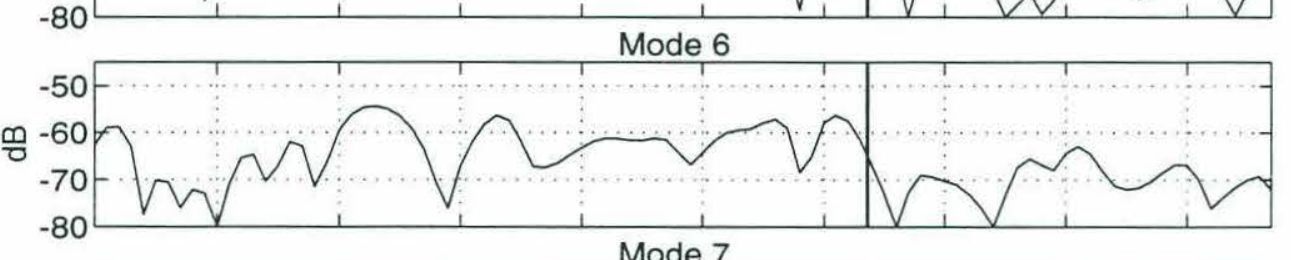

$-603$
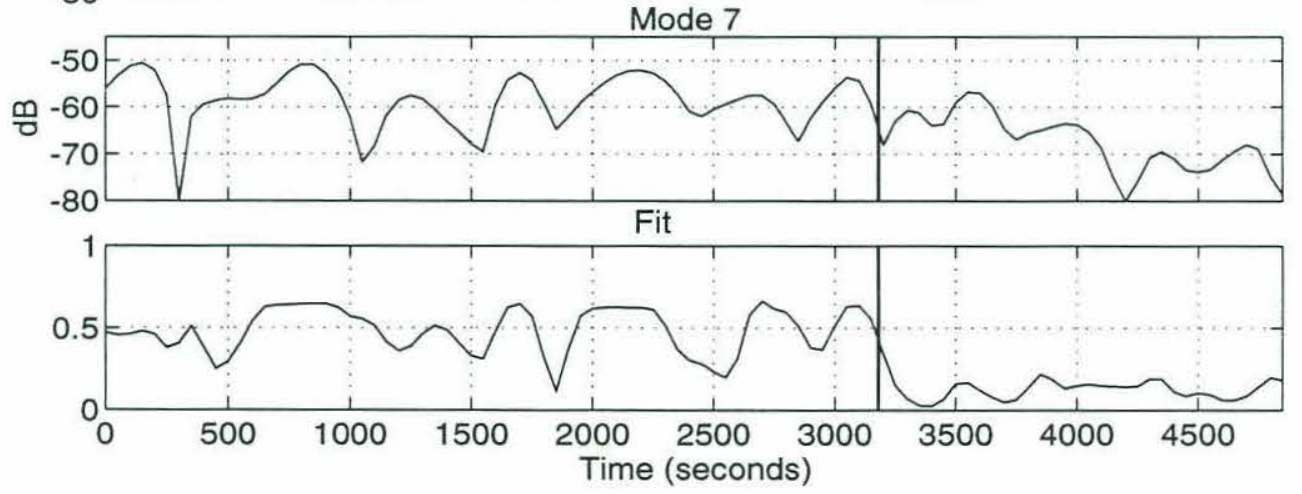

Figure 4-13: Estimated mode coefficients for Event 01261525. Assumed orientation of $1.5^{\circ}$ tilt and relative azimuth to incoming signal of $100^{\circ}$. (20\% diagonal loading) Mean and standard deviations during signal duration are shown at right. 


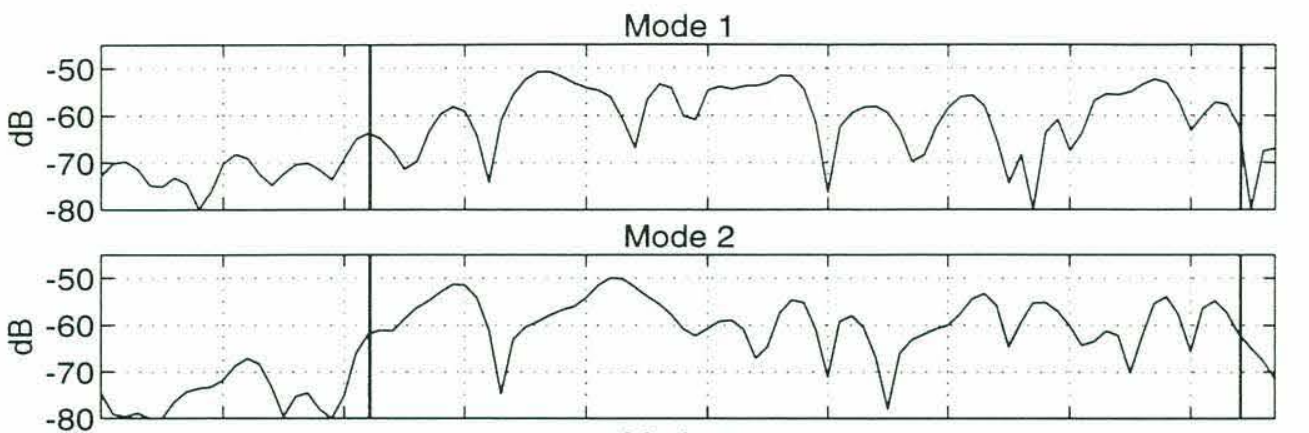

Mean S.D.

$(\mathrm{dB})$

$-553$

$-553$

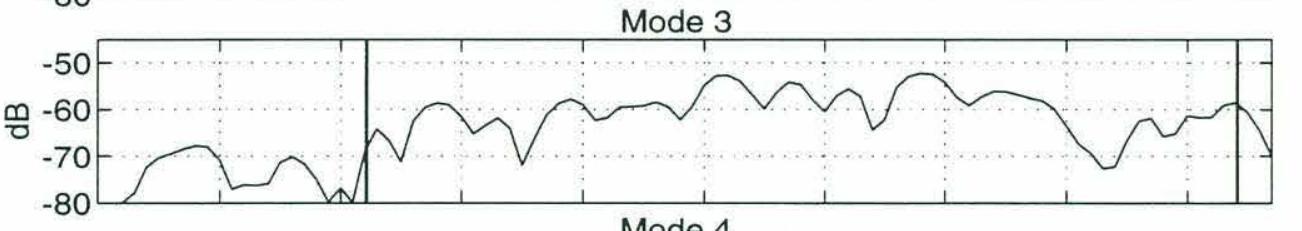

$-60 \quad 2$

Mode 4

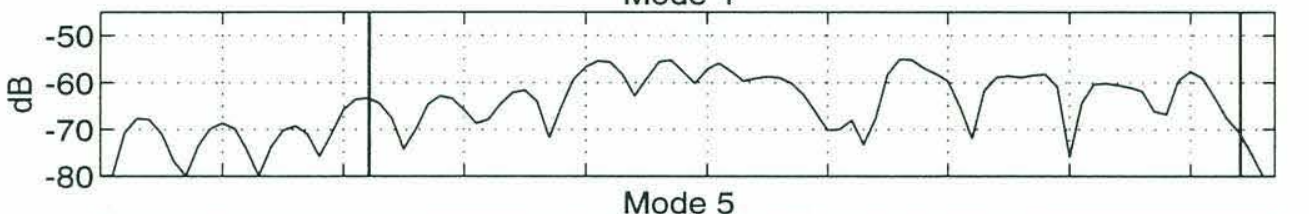

$-592$

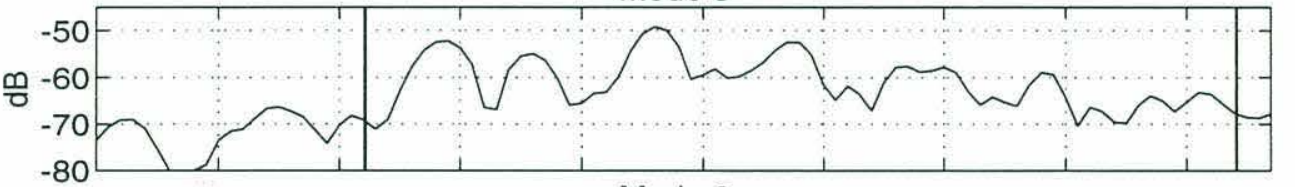

$-553$

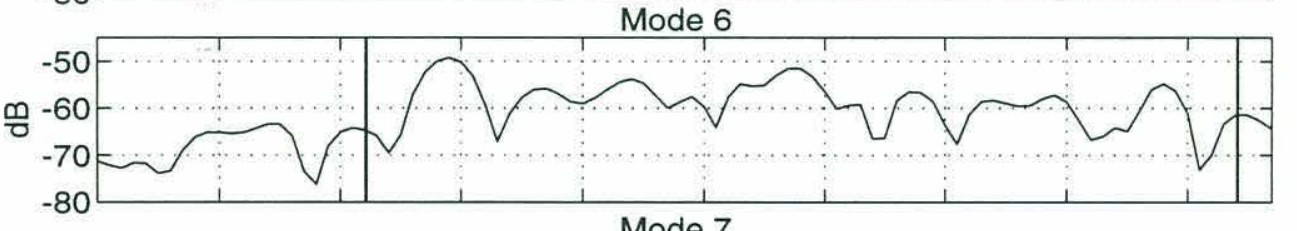

$-562$
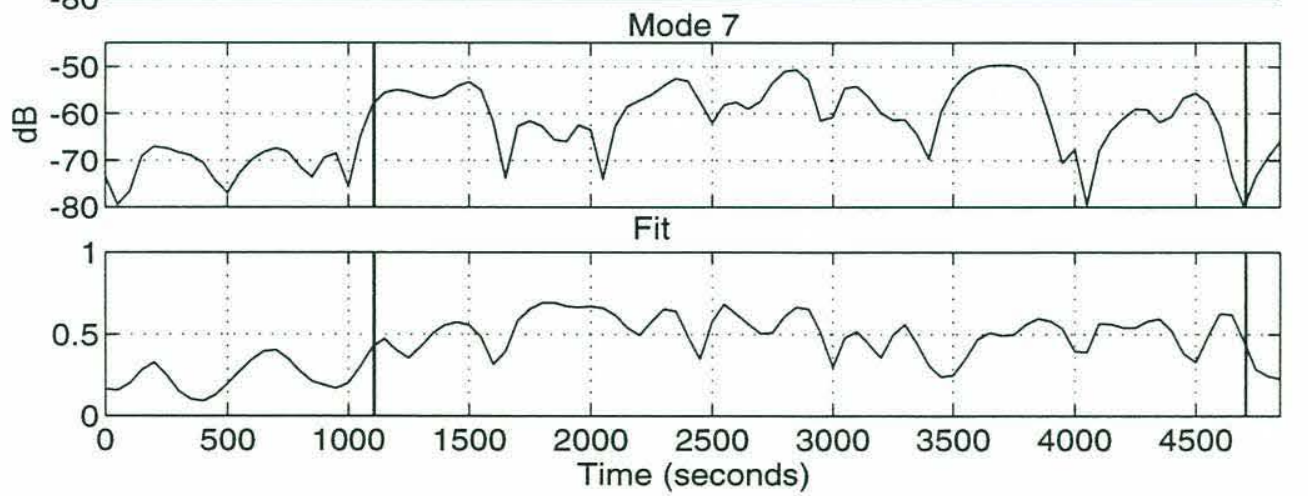

Figure 4-14: Estimated mode coefficients for Event 01270322. Assumed orientation was a $2.5^{\circ}$ tilt and relative azimuth to incoming signal of $30^{\circ}$. (20\% diagonal loading) Mean and standard deviations during signal duration are shown at right. 


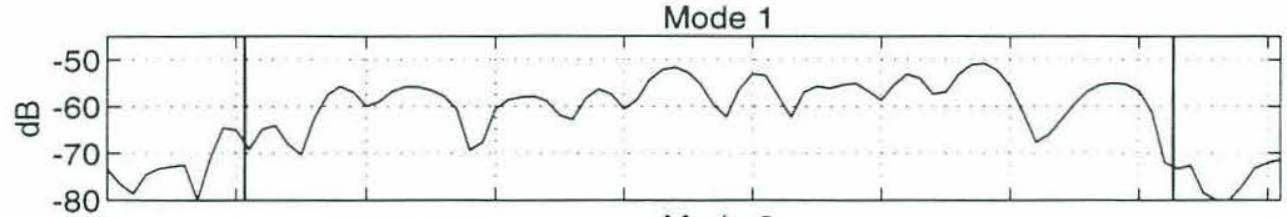

Mean S.D.

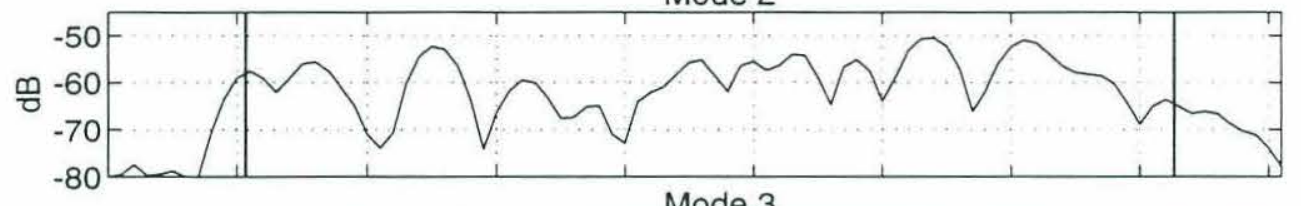

$-57 \quad 6$

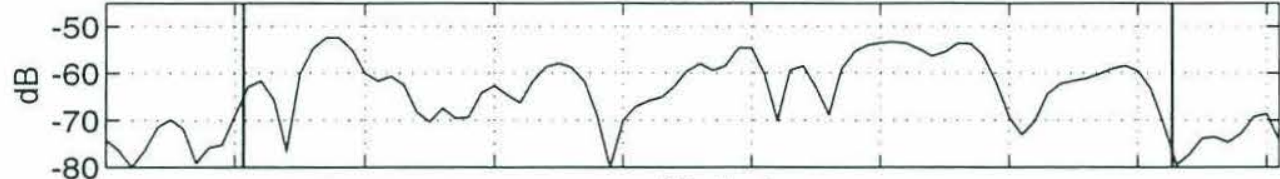

$-58 \quad 6$

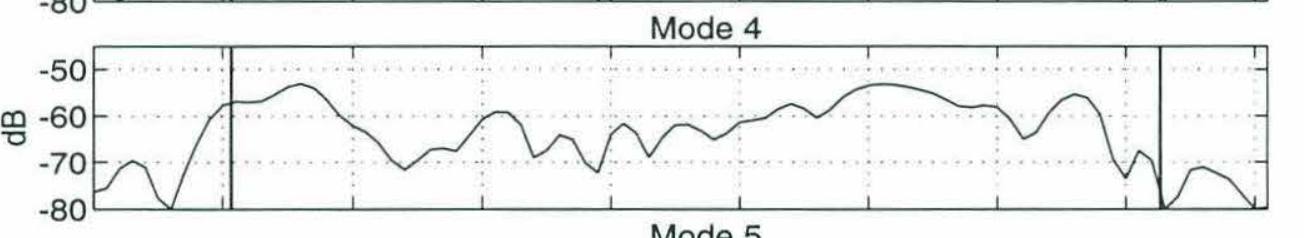

$-59 \quad 5$
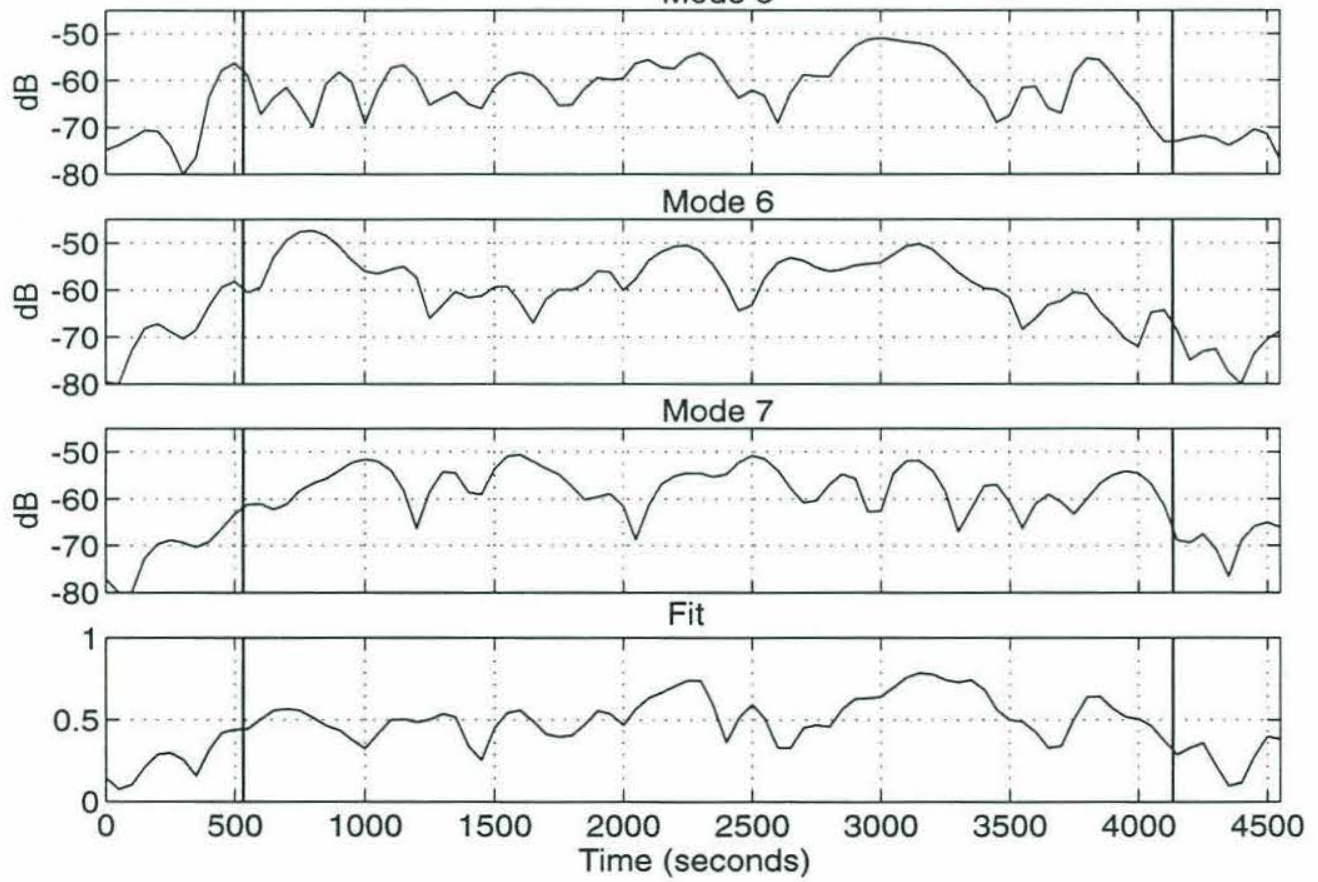

$-554$

Figure 4-15: Estimated mode coefficients for Event 01271505. Assumed orientation of $1.5^{\circ}$ tilt and relative azimuth to incoming signal of $60^{\circ}$. (20\% diagonal loading) Mean and standard deviations during signal duration are shown at right. 


\section{Chapter 5}

\section{Conclusions}

\subsection{Summary}

Three of the continuous-wave transmission events recorded by the vertical array off the coast of Monterey during the 1991 Heard Island Feasibility Test have been processed and analyzed. In particular, the analysis focused on determining the modal content, or vertical structure, of the signal. As covered in Chapter 1, knowing the vertical signal structure is important in determining how the signals propagated through the ocean.

The recorded data were characterized by very low signal levels, large transient spikes, and a limited set of operational channels. A conditioning scheme was developed to improve the quality of the data and to simplify the processing. This consisted of a spike suppression routine, followed by a quadrature demodulation and downsampling, and then very narrowband lowpass filtering. The input signal-tonoise ratio was $-15 \mathrm{~dB}$ on a single hydrophone, and the narrow filtering provided a $25 \mathrm{~dB}$ increase in the SNR.

Because of movement of the source ship, the data acquired a slight Doppler shift. As discussed in Chapter 2, it was possible to accurately predict the Doppler shift 
for each of the transmissions. The predicted and measured shifts were within \pm 2 $\mathrm{mHz}$ of each other. Using the measured shift, the launch angle at the source was calculated and found to be in very close agreement with that predicted by models prior to HIFT.

In Chapter 3, the least squares modal beamformer was presented and contrasted to the simpler, single-mode beamformer. The main issue of interest was how the two processors compared when the modeshapes were sub-sampled. The advantage of the least squares method was that it reduced the leakage from adjacent modes. However, loss of mode orthogonality due to the sub-sampling created singularity problems for the singular value decomposition. This was remedied by the addition of diagonal loading, or damping. A relatively large value of $20 \%$ loading was found to be necessary to control the singularities.

The major contribution of this work was the modal analysis of the datasets. This was accomplished by using sonograms to show the signal energy distribution $v s$ depth, and by using a least squares decomposition to show the contribution from each mode. It was shown that the modal content of the signal, after having propagated nearly half-way around the world, was greater than predicted. These results are support by the results of other independent analyses of the same datasets, as well as modeling efforts done after HIFT.

\subsection{Conclusions}

In retrospect, the complexity of the signal structure off Monterey is not that surprising. Following HIFT, much effort was put into explaining and modeling the results, not only those seen off Monterey but at the other receiver sites as well. A number of

factors could possibly account for the observed structure. Propagation through an oceanic front such as the Antarctic Convergence Zone will redistribute the energy be- 
tween the modes. Going over the Campbell Plateau, modes 10 and above are likely stripped away, leaving a signal structure much like what was seen in the analysis here. Another potential explanation is the accumulated effects of modal scattering from internal waves or other small-scale features. This amounts to small transfers of energy between modes that, over very long distances, can significantly alter the energy distribution.

It is unfortunate that the data quality did not allow a more extensive analysis. Both the low signal levels and the lack of precise array orientation data complicated the analysis. With higher signal levels, it would have been possible to look at the other two signal types in addition to the continuous-wave events. More knowledge of the array orientation would have allowed all 21 of the usable sensors to be used in the modal beamforming. The orthogonality would be greatly improved and the least squares decomposition could have been extended to include modes beyond mode 7 . There would also be less leakage, and relative amplitude estimates would be more accurate. This would be especially useful for making comparisons with the postHIFT modeling results.

\subsection{Future Work}

There is still a significant amount of work that needs to be done before the physics of very long range propagation are completely understood, and before acoustic thermometry can be used in climate monitoring. The preliminary results from modeling by Baggeroer [26] suggest that modal coupling due to internal waves may very well be a dominant factor in determining the modal distribution at long ranges. One of the major issues under consideration is how the ocean processes, including internal waves, should be characterized and how they actually couple into the acoustics. How these are modeled significantly impacts the accumulated effects over long ranges. In 
addition to this work, data will soon be available from a second experiment, known as Acoustic Thermometry of Ocean Climate (ATOC). ATOC has been specifically designed to eliminate many of the factors that complicated the analysis of the Heard Island data. Transmissions along paths that avoid polar waters, oceanic fronts, and major bathymetry, will play a crucial role in isolating the effects of internal waves on mode coupling. 


\section{Bibliography}

[1] Walter H. Munk and Andrew M.G. Forbes. Global ocean warming: An acoustic measure? J. Phys. Oceanogr., 19:1765, November 1989.

[2] Uwe Mikolajewicz, Benjamin Santer, and Ernst Maier-Reimer. Ocean response to greenhouse warming. Nature, 345:89, June 1990.

[3] Walter H. Munk, Robert C. Spindel, Arthur B. Baggeroer, and Theodore G. Birdsall. The Heard Island Feasibility Test. J.Acoust.Soc.Am., 1993. (submitted).

[4] R.C. Shockley, J. Northrop, P.G. Hansen, and C. Hartdegen. SOFAR propagation paths from Australia to Bermuda: Comparison of signal speed algorithms and experiments. J.Acoust.Soc.Am., 71, 1982.

[5] John Spiesberger, Paul Bushong, Kurt Metzger, and Theodore Birdsall. Ocean acoustic tomography: Estimating the acoustic travel time with phase. IEEE J. of Oceanic Engineering, 14(1), January 1989.

[6] Ching-Sang Chiu, Albert J. Semtner, Coenradd M. Ort, James H. Miller, and Laura L. Ehret. A ray variability analysis of sound transmission from Heard Island to California. J.Acoust.Soc.Am., 1993. (submitted).

[7] Arthur B. Baggeroer, Khosrow Lashkari, Ching-Sang Chiu, James H. Miller, Peter Mikhalevsky, and Keith von der Heydt. Vertical array receptions of the Heard Island transmissions. J.Acoust.Soc.Am., 1993. (submitted).

[8] K.D. Heaney, W.A Kuperman, and B.E. McDonald. Perth-Bermuda sound propagation (1960): Adiabatic mode interpretation. J.Acoust.Soc.Am., 90, November 1991.

[9] L.M. Brekhovskikh and Yu.P. Lysanov. Fundamentals of Ocean Acoustics. Springer-Verlag, Berlin, Second edition, 1990.

[10] Clarence S. Clay and Herman Medwin. Acoustical Oceanography:Principles and Applications. Wiley-Interscience, New York, 1977. 
[11] Ivan Tolstoy and C.S. Clay. Ocean Acoustics: Theory and Experiment in Underwater Sound. American Institute of Physics, New York, 1987.

[12] D. Michael Milder. Ray and wave invariants for SOFAR channel propagation. J.Acoust.Soc.Am., 46(5), November 1969.

[13] Peter F. Worcester, Bruce D. Cornuelle, and Robert C. Spindel. A review of acoustic tomography: 1987-1990. Reviews of Geophysics, Supplement, pages 557-570, April 1991.

[14] Keith von der Heydt. An optical disk based data acquisition system (ODAS). Technical report, Woods Hole Oceanographic Institution, November 1987.

[15] Andrew Forbes and Walter Munk. Doppler-inferred launch angles of global acoustic ray paths. J.Acoust.Soc.Am., 1993. (submitted).

[16] M. Dzieciuch and W. Munk. Differential doppler as a diagnostic. J.Acoust.Soc.Am., 1993. (submitted).

[17] Keith von der Heydt. personal communications.

[18] Garry J. Heard and N.R. Chapman. Heard Island Feasibility Trial: Analysis of Pacific path data obtained with a horizontal line array. J.Acoust.Soc.Am., 1993. submitted.

[19] Theodore Birdsall, Kurt Metzger, and Matthew Dzieciuch. HIFT signals, signal processing, and general results. J.Acoust.Soc.Am., 1993. submitted.

[20] John Polcari. Modal Beamforming with Arctic Data. PhD thesis, Massachussetts Institute of Technology, 1986.

[21] T.C. Yang. A method of range and depth esimation by modal decomposition. J.Acoust.Soc.Am., 82, November 1987.

[22] L. Lawson and R.J. Hansen. Solving Least Squares Problems. Prentice Hall, Englewood Cliffs, NJ, 1974.

[23] William Menke. Geophysical Data Analysis: Discrete Inverse Theory. Academic Press, Inc., San Diego, 1989.

[24] Arthur B. Baggeroer. Course notes - Geophysical and Oceanographic Signal Processing II, Spring 1993.

[25] Arthur B. Baggeroer. A numerical approach to the solution of acoustic wave equations. still in draft form. 
[26] Arthur B. Baggeroer. personal communications.

[27] B. E. McDonald, M. D. Collins, W. A. Kuperman, and K. D. Heaney. Comparison of data and model predictions for Heard Island acoustic transmissions. J.Acoust.Soc.Am., 1993. 